\title{
The (w)hole survey: An unbiased sample study of transition disk candidates based on Spitzer catalogs ${ }^{\star}$
}

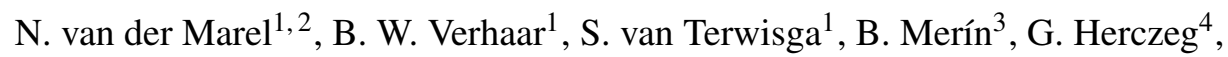 \\ N. F. W. Ligterink ${ }^{1}$, and E. F. van Dishoeck ${ }^{1,5}$
}

\author{
${ }^{1}$ Leiden Observatory, Leiden University, PO Box 9513, 2300 RA Leiden, The Netherlands \\ e-mail: nmarel@strw.leidenuniv.nl \\ ${ }^{2}$ Institute for Astronomy, University of Hawaii, 2680 Woodlawn Drive, Honolulu HI 96822, USA \\ 3 European Space Astronomy Centre (ESA), PO Box 78, 28691 Villanueva de la Cañada, Spain \\ ${ }^{4}$ Max-Planck-Institut für Extraterrestrische Physik, Giessenbachstrasse 1, 85748 Garching, Germany \\ ${ }^{5}$ Kavli institute, Peking University, 100871 Beijing, PR China
}

Received 5 January 2016 / Accepted 23 March 2016

\begin{abstract}
Understanding disk evolution and dissipation is essential for studies of planet formation. Transition disks, i.e., disks with large dust cavities and gaps, are promising candidates of active evolution. About two dozen candidates, selected by their spectral energy distribution (SED), have been confirmed to have dust cavities through millimeter interferometric imaging, but this sample is biased toward the brightest disks. The Spitzer surveys of nearby low-mass star-forming regions have resulted in more than 4000 young stellar objects. Using color criteria, we selected a sample of $\sim 150$ candidates and an additional 40 candidates and known transition disks from the literature. The Spitzer data were complemented by new observations at longer wavelengths, including new JCMT and APEX submillimeter photometry, and WISE and Herschel-PACS mid- and far-infrared photometry. Furthermore, optical spectroscopy was obtained and stellar types were derived for $85 \%$ of the sample, including information from the literature. The SEDs were fit to a grid of RADMC-3D disk models with a limited number of parameters: disk mass, inner disk mass, scale height and flaring, and disk cavity radius, where the latter is the main parameter of interest. About $72 \%$ of our targets possibly have dust cavities based on the SED. The derived cavity sizes are consistent with imaging/modeling results in the literature, where available. Trends are found with $L_{\text {disk }}$ over $L_{*}$ ratio and stellar mass and a possible connection with exoplanet orbital radii. A comparison with a previous study where color observables are used reveals large overlap between their category of planet-forming disks and our transition disks with cavities. A large number of the new transition disk candidates are suitable for follow-up observations with ALMA.
\end{abstract}

Key words. protoplanetary disks - planets and satellites: formation - planet-disk interactions

\section{Introduction}

A central question in planet formation is how the optically thick protoplanetary disks around classical $\mathrm{T}$ Tauri stars evolve into the optically thin debris disks around older systems (Williams \& Cieza 2011). An important part of the evolution occurs in the transitional phase between these two regimes. Transitional disks, i.e., disks with inner dust cavities, are considered to form the evolutionary link, although it remains uncertain whether all disks go through this phase at some point during their lifetime (e.g., Cieza et al. 2007; Currie \& Kenyon 2009). One of the most exciting explanations for transition disks is the presence of a young planet that has cleared out its orbit (Lin \& Papaloizou 1979). This scenario has been confirmed through the (tentative) detection of planets embedded in transition disks through direct imaging for a handful of disks (Kraus \& Ireland 2012; Quanz et al. 2013; Reggiani et al. 2014; Quanz 2015; Sallum et al. 2015). As it remains unclear how and at what stage planets are formed in a disk, finding them at the earliest stage and study of their environment can provide important clues on the planet formation process. For a better understanding

^ Full Tables 4, 5, A.1-A.3, C.1, and D.1 are only available at the CDS via anonymous ftp to

cdsarc.u-strasbg. fr (130.79.128.5) or via

http://cdsarc.u-strasbg.fr/viz-bin/qcat?J/A+A/592/A126 of the role of transition disks in the disk evolution and planet formation process, a large unbiased sample of transition disks with large holes should be studied.

The transition disk fraction is thought to be $5-25 \%$ depending on the definition with the fraction varying with stellar age (Currie \& Sicilia-Aguilar 2011), implying that the evolutionary path through a transition disk is either rapid or uncommon. Transitional disk candidates are traditionally identified through a deficit of infrared flux in the mid-IR spectral energy distribution (SED; e.g., Strom et al. 1989; Calvet et al. 2002; Espaillat et al. 2014, for review ). The deficit arises from the absence of hot small dust particles close to the star, which can be caused by either grain growth (e.g., Dullemond \& Dominik 2005), photoevaporative clearing (e.g., Alexander et al. 2006), or interaction with a stellar companion or recently formed planet (e.g., Artymowicz \& Lubow 1994), which are all processes closely linked to disk evolution. Thanks to Spitzer mid-infrared spectroscopy surveys, a large number of transitional disks has been discovered through a minimum in the infrared part of their SED (e.g., Brown et al. 2007; Najita et al. 2007; Kim et al. 2009; Merín et al. 2010). Submillimeter observations of about two dozen of the brightest disks have directly resolved large holes with pioneering interferometers, confirming their transition disk status (e.g., Piétu et al. 2005; Brown et al. 2008, 2009; Isella et al. 2010a,b; Andrews et al. 2011). The hole 
sizes generally match well with estimates from SED modeling, suggesting that the current interpretation and modeling of SEDs can correctly infer this parameter provided that the mid-infrared part of the SED is well covered observationally. The Atacama Large Millimeter/submillimeter Array (ALMA) has produced even sharper dust images of a small sample of transition disks with evidence for dust trapping (van der Marel et al. 2013; Casassus et al. 2013; Pérez et al. 2014; Zhang et al. 2014). Also, ALMA has revealed the gas distribution through CO observations, which show that substantial amounts of gas are present inside the dust cavities (Bruderer et al. 2014; van der Marel et al. 2015, 2016; Perez et al. 2015), indicating the presence of planets. However, ALMA has so far focused on the most well-studied and brightest transition disks. A larger sample is required to derive a more general picture.

Transition disk candidates have historically been identified through a range of different criteria (Brown et al. 2007; Muzerolle et al. 2010; Oliveira et al. 2010; Merín et al. 2010; Cieza et al. 2010, 2012b; Romero et al. 2012), usually involving the Spitzer colors in the mid-infrared. The availability of Spitzer IRS spectra between 5-35 $\mu \mathrm{m}$ was crucial for classification and determination of the hole size in these studies especially in covering the $8-20 \mu \mathrm{m}$ region where the SEDs reach their minimum but is not well covered by the 8 and 24 micron photometry points. In recent years, far-infrared Herschel-PACS and SPIRE photometry has been used to identify and characterize (transition) disks (e.g., Ribas et al. 2013; Bustamante et al. 2015; Rebollido et al. 2015). Other studies identified candidates by comparing the infrared part of their SEDs with the median T Tauri disk SED (e.g., Harvey et al. 2007; Merín et al. 2008). These studies define a separate class of transition disks as socalled anemic disks: disks with homologous depletion of dust because of grain growth or settling at all radii, exhibiting a lowinfrared excess at all wavelengths. Furthermore, some studies distinguish between pretransitional and transitional disks: disks with a gap (inner disk present inside the cavity) and disks with a hole (Espaillat et al. 2007), although there is no obvious evolutionary connection. A so-called cold disk (Brown et al. 2007) refers to a transition disk with a strong deficit in the mid-infrared, implying a cavity with a steep inner wall. A few transition disks have been found in millimeter imaging without evidence for mid-infrared dips in their SED, for example, MWC 758 (Isella et al. 2010b)

Selection of candidates is sometimes followed up by radiative transfer modeling of the radial disk structure to constrain the dust cavity size and disk mass (Kim et al. 2009; Merín et al. 2010) and thereby determine the origin of the cavity other than through clearing by a companion. Increased grain growth in the inner part of disk would result in the appearance of a dust deficit in the SED (Dullemond \& Dominik 2005), although this would not be visible in millimeter imaging (Birnstiel et al. 2010). Furthermore, multiplicity studies can define the origin of the cavity as circumbinary disk, whereas measuring the accretion through optical $\mathrm{H} \alpha$ can determine photoevaporative clearing (Najita et al. 2007; Espaillat et al. 2007; Cieza et al. 2010). Theoretical work has also shown that photoevaporative clearing cannot explain the largest observed cavities and a combination of processes may be responsible (Owen \& Clarke 2012; Rosotti et al. 2013).

Overall, the definition of a transition disk candidate remains loose and has been used in various contexts in different studies. As a result of a lack of a large sample of transition disks, general properties remain uncertain and it is still unclear whether the origin for all transition disk cavities is the same or whether disks follow different evolutionary paths (Cieza et al. 2007). Also, the distribution of cavity radii is not known, and this could constrain the birth sites of giant planets before migration. The analysis of a large unbiased sample of transition disks and candidates can provide firm constraints on their general properties. Spitzer surveys in all nearby $(<500 \mathrm{pc})$ star-forming regions (Cores to Disks (c2d), Gould-Belt (GB), and Taurus) have provided identification and SEDs of several thousands of young stellar objects (YSOs; e.g., Evans et al. 2009; Rebull et al. 2010; Dunham et al. 2015, and references therein), out of which there are many transition disk candidates. In addition, in recent years the AllWISE catalog of mid-infrared targets has become available (Wright et al. 2010), and the Herschel Space Observatory (Pilbratt et al. 2010) has observed large parts of nearby starforming regions in the far-infrared. Owing to the availability of Spitzer data combined with WISE and Herschel data, the timing is perfect for a large SED survey of transition disks.

In this work, we analyze transition disk candidates selected from the Spitzer catalogs using robust color criteria developed by Merín et al. (2010). These criteria were developed after deep analysis of the SEDs including IRS spectra. Our sample is complemented by additional candidates and known transition disks from the literature. The SEDs are complemented with optical, new archival far-infrared Herschel, Spitzer IRS spectra (where available), and new submillimeter observations and are modeled using the dust radiative transfer code RADMC-3D with a generic disk structure with a cavity. The main parameter of interest is the cavity size $r_{\text {cav }}$. In Sect. 2 we discuss the selection criteria of the sample and additional observations, Sect. 3 presents the results of the observations, Sect. 4 discusses the modeling procedure and limitations and the resulting disk parameters, and in Sect. 5 we discuss the robustness of the sample and comparison with previous studies. One of the aims of this study is to define a large sample of transition disk candidates with dust cavities that are large enough to be imaged in the future by ALMA $(\geq 10 \mathrm{AU}$ or $\sim 0.03^{\prime \prime}$, for the largest distances). The resolved images of gas and dust provide more clues concerning the origin of the dust cavities and the place of transition disks in disk evolution.

\section{Observations}

\subsection{Target selection}

The c2d, GB and Taurus Spitzer Legacy programs completed full infrared surveys using the Infrared Array Camera (IRAC, 3.6-8.0 $\mu \mathrm{m}$ ) and Multiband Imaging Photometer (MIPS, $24-160 \mu \mathrm{m})$ in the nearby star-forming regions ( $\leq 450 \mathrm{pc})$, resulting in more than 3000 identified YSOs (see Table 1 for an overview of papers presenting the data). Several bright YSOs from the $\mathrm{c} 2 \mathrm{~d}$ survey were targeted for additional observation with the Spitzer InfraRed Spectrograph (IRS, 5-35 $\mu \mathrm{m}$ ). Merín et al. (2010, hereafter M10) analyzed 35 possible transition disk candidates, for which IRS spectra were available in detail through SED modeling, and defined two sets of color criteria as follows:

$$
\begin{aligned}
{[\mathrm{A}]: 0.0<[3.6]-[8.0] } & <1.1, \\
3.2<[8.0]-[24.0] & <5.3, \\
{[\mathrm{~B}]: 1.1<[3.6]-[8.0] } & <1.8, \\
3.2<[8.0]-[24.0] & <5.3,
\end{aligned}
$$

where the bracketed numbers refer to the magnitudes at the Spitzer wavelengths. The Region A criteria select clean inner holes (disks for which there is no substantial excess in any IRAC 
Table 1. Overview Spitzer papers of YSOs in star-forming regions.

\begin{tabular}{llll}
\hline \hline & \multicolumn{1}{c}{ Cores to Disks (c2d) } & $\mathrm{d}(\mathrm{pc})$ & Paper \\
\hline Ophiuchus (MIPS) & Padgett et al. (2008) & 120 & VII \\
Serpens & Harvey et al. (2007) & $250-400^{a}$ & IX \\
Cham II & Alcalá et al. (2008) & 180 & X \\
Lupus I,III,IV & Merín et al. (2008) & $150-200$ & XI \\
Perseus & Young et al. (2015) & 250 & XII \\
WTTS (c2d) & Padgett et al. (2006), Cieza et al. (2007), Wahhaj et al. (2010) & - & \\
Disks with holes (c2d) & Merín et al. (2010) & - & \\
\hline \multicolumn{1}{c}{ Gould Belt (GB) } & & I \\
\hline IC 5146 & \multicolumn{1}{c}{ Oarvey et al. (2008) } & 950 & II \\
Cepheus & Kirk et al. (2009) & 300 & III \\
CrA & Peterson et al. (2011) & 150 & IV \\
Lupus V \& VI (full) & Spezzi et al. (2011) & 150 & V \\
Ophiuchus North & Hatchell et al. (2012) & 450 & VI \\
Auriga & Broekhoven-Fiene et al. (2014) & & \\
\hline \multicolumn{1}{c}{ Others } & 97 & \\
\hline Cham (IRAC) & Megeath et al. (2005) & 97 & 160 \\
$\eta$ Cham (MIPS) & Sicilia-Aguilar et al. (2009) & 140 & \\
Cham I & Luhman et al. (2008) & 450 & \\
Taurus & Rebull et al. (2010), Luhman et al. (2010) & 450 & \\
Orion & Hernández et al. (2010) & - & \\
FEPS & Megeath et al. (2012) & Carpenter et al. (2008) &
\end{tabular}

Notes. ${ }^{(a)}$ The distance to Serpens is uncertain, but recent VLBA observations put it at $415 \mathrm{pc}$ (Dzib et al. 2011), which has been used in this study.

band and there is a clear signature of an inner dust hole) and the Region B criteria select disks with a clear signature of an inner dust hole, but some excess in the IRAC bands, possibly resulting from an inner disk. The latter criterion includes several of the confirmed imaged transition disks (Brown et al. 2009; Andrews et al. 2009), but may also include some disks without holes (M10).

M10 finds one transition disk with a particularly large hole (Sz 84, object 17), which falls outside of the color criteria mentioned above. Inspection of its SED reveals a steep slope between the $24 \mu \mathrm{m}$ and $70 \mu \mathrm{m}$ flux. Therefore, we set an additional color criterium, i.e.,

$$
\begin{aligned}
{[\mathrm{L}]: 0.0 } & <[3.6]-[8.0]<1.1 \\
10.0 & >[24.0]-[70.0]>3.8 .
\end{aligned}
$$

In this case the MIPS-2 flux at $70 \mu \mathrm{m}$ has to be detected rather than an upper limit. Because of the large beam size of Spitzer at $70 \mu \mathrm{m}$ of $18 "$, this flux can be confused with nearby sources. The interpretation of the long wavelength flux has to be done carefully for the disks selected by the Region L criteria. The Region L targets are not mutually exclusive from the Region A criteria, some targets appear in both.

The color criteria were applied to the three main Spitzer catalogs, listed in Table 2, resulting in 153 candidates. In addition to the catalogs, we searched the literature for additional transition disk candidates, using the color criteria on Spitzer targets that were not included in the catalogs (row "Other" in Table 2), finding an additional 12 disks. Targets in Orion, Cepheus (Kirk et al. 2009), and IC 5146 (Harvey et al. 2008) are not included because of their large distances (450, 500, and $950 \mathrm{pc}$, respectively). Finally, we added seven confirmed transition disks known from resolved millimeter imaging and 21 targets that were denoted as transition disk candidates by various
Table 2. Target selection in each catalog.

\begin{tabular}{l|lll}
\hline \hline Catalog/Criterion & [A] & [B] & [L] \\
\hline c2d (Evans et al. 2009) & 30 & 34 & 9 \\
GB (Dunham et al. 2015) & 25 & 15 & 31 \\
Taurus (Rebull et al. 2010) & 7 & 12 & 6 \\
Other samples $^{a}$ & 7 & 4 & 1 \\
\hline Additional targets $^{b}$ & \multicolumn{3}{|c}{7 imaging } \\
& \multicolumn{3}{|c}{ 21 SED } \\
\hline
\end{tabular}

Notes. ${ }^{(a)}$ Targets were selected using our color criteria in the following papers, for targets not included in the c2d/GB/Taurus catalogs: Padgett et al. (2006), Silverstone et al. (2006), Carpenter et al. (2008), Luhman et al. (2008), Kim et al. (2009), Sicilia-Aguilar et al. (2009), Cieza et al. (2010), Luhman et al. (2010). (b) Some targets were added from the literature that did not follow the color criteria. Imaging targets were taken from Piétu et al. (2006), Ohashi (2008), Brown et al. (2009), Isella et al. (2010a), Andrews et al. (2010, 2011), Rosenfeld et al. (2013), van der Marel et al. (2013). The other targets were identified as transition disk candidates by Megeath et al. (2005), Hernández et al. (2007), Merín et al. (2008), Monnier et al. (2008), Hughes et al. (2008), Sicilia-Aguilar et al. (2008), Ireland \& Kraus (2008), Kim et al. (2009), McClure et al. (2010), Najita et al. (2010), Espaillat et al. (2011), Furlan et al. (2011).

authors, but were not yet included by the color criteria. The number of targets from various selections are listed in Table 2 with corresponding references. All targets in the sample are listed in Table A.1. Several of the color-selected targets have been identified as transition disk candidates or confirmed by millimeter imaging, as indicated in the last column of Table A.1.

The distance to Serpens is uncertain with values between 250 and $400 \mathrm{pc}$ (discussion in, e.g., Oliveira et al. 2009). However, VLBA observations have set a distance of $415 \mathrm{pc}$ for the main 
cloud (Dzib et al. 2010), which has been used in more recent work (Erickson et al. 2015; Ortiz-León et al. 2015), and has also been used in this study.

\subsection{Additional photometry}

For each target, an SED was constructed using the Spitzer IRAC and MIPS photometry, complemented with optical $B, V$, and $R$ data from the NOMAD catalog (Zacharias et al. 2005) and near-infrared $J, H$, and $K$ photometry from 2MASS (Cutri et al. 2003). Reduced Spitzer IRS low-resolution spectra of 5-35 $\mu \mathrm{m}$ were taken from the Cornell Atlas of Spitzer/IRS Sources (CASSIS) (Lebouteiller et al. 2011) when available. For ID63 (DoAr28), the IRS spectrum in CASSIS included extended emission, a properly reduced spectrum was kindly provided by Melissa McClure (McClure et al. 2010). Unfortunately, IRS spectra are not available for the entire sample, while colors only provide limited constraints on the derived cavity size. Bright isolated targets could be complemented with IRAS photometry, especially when Spitzer data were saturated. The Wide-field Infrared Survey Explorer (WISE) performed an all-sky survey in four wavelength bands: $3.4,4.6,12.0$, and $22 \mu \mathrm{m}$, leading to the AllWISE Source Catalog (Wright et al. 2010). The coordinates of the targets in our sample were matched with the WISE targets (within $2^{\prime \prime}$ ) and the fluxes were added to the SEDs. Although three of the four bands overlap with Spitzer, the $12 \mu \mathrm{m}$ flux provides an important data point in between IRAC and MIPS wavelengths when no IRS spectra are available. Furthermore, the diffraction limited beam size of the WISE satellite is twice as large as the Spitzer beam (see Table 3). The comparison between the WISE $22 \mu \mathrm{m}$ flux with the MIPS-1 $24 \mu \mathrm{m}$ flux provides an independent check of confusion at longer wavelengths, if the $22 \mu \mathrm{m}$ flux is much larger, there is likely to be a nearby source that contributes to the $70 \mu \mathrm{m}$ MIPS-2 flux as well. Although the Spitzer c2d and GB catalogs provide a quality flag on the MIPS-2 flux (MP2_Q_det_c) for possible confusion, this independent alternative check showed which targets were confused at longer wavelengths more directly. A difference between the 22 and $24 \mu \mathrm{m}$ flux could also originate from infrared variability, for example, owing to scale height changes in the inner disk (e.g., Flaherty \& Muzerolle 2010; Espaillat et al. 2011). However, such variability is typically on the order of $20-40 \%$. Therefore, we only consider confusion if the difference in flux is more than $50 \%$. The fluxes of different telescopes are taken with years in between, so without infrared monitoring there is no possibility of quantifying this effect for the targets in our sample, but the effect on our SED modeling is expected to be minor. The following targets were removed from the sample because of possible confusion, and their SEDs were not analyzed further: IDs $30,32,82,85,86,88,90,92,93,95,97,98,116,123,126$, 202, 346, and 347 .

At longer wavelengths, the SEDs were complemented with (sub)millimeter data from the literature where available (see refs in Table C.1). A subsample of the remaining targets were observed with the James Clerk Maxwell Telescope (JCMT) ${ }^{1}$ and the Atacama Pathfinder Experiment (APEX) ${ }^{2}$. Targets were

\footnotetext{
1 The James Clerk Maxwell Telescope has historically been operated by the Joint Astronomy Centre on behalf of the Science and Technology Facilities Council of the United Kingdom, the National Research Council of Canada, and the Netherlands Organisation for Scientific Research Additional funds for the construction of SCUBA-2 were provided by the Canada Foundation for Innovation.

2 This publication is based on data acquired with the Atacama Pathfinder Experiment (APEX). APEX is a collaboration between the
}

Table 3. Beam sizes and apertures for photometry.

\begin{tabular}{l|lll}
\hline \hline Telescope & Instrument & $\begin{array}{l}\text { Wavelength } \\
\text { range }(\mu \mathrm{m})\end{array}$ & $\begin{array}{l}\text { Beam size/ } \\
\text { Aperture }\left(^{\prime \prime}\right)\end{array}$ \\
\hline Spitzer & IRAC & $3.6,4.5,5.8,8.0$ & $1.7-1.9$ \\
& MIPS & $24.0,70.0$ & $6.0,18$ \\
WISE & & $3.4,4.6,12,22$ & $6.1,6.4,6.5,12$ \\
Herschel & PACS & $70,100,160$ & $5.5,6.5,11$ \\
APEX & SABOCA & 350 & 7.8 \\
& LABOCA & 870 & 19 \\
JCMT & SCUBA-2 & 850 & 15 \\
\hline
\end{tabular}

Table 4. APEX photometry at 350 and $870 \mu \mathrm{m}$ for our sample.

\begin{tabular}{lll|lll}
\hline \hline ID & $\begin{array}{l}F_{350 \mu \mathrm{m}} \\
(\mathrm{Jy})\end{array}$ & $\begin{array}{l}F_{870 \mu \mathrm{m}} \\
(\mathrm{mJy})\end{array}$ & ID & $\begin{array}{l}F_{350 \mu \mathrm{m}} \\
(\mathrm{Jy})\end{array}$ & $\begin{array}{l}F_{870 \mu \mathrm{m}} \\
(\mathrm{mJy})\end{array}$ \\
\hline 1 & $2.4 \pm 0.2$ & $210 \pm 20$ & 40 & $<0.7$ & $<20$ \\
2 & - & $<40$ & 43 & - & $<18$ \\
6 & $0.69 \pm 0.18$ & & 46 & $0.8 \pm 0.2$ & $164 \pm 14$ \\
9 & - & $<15$ & 55 & $<0.9$ & $92 \pm 6$ \\
10 & - & $<20$ & 58 & - & $<18$ \\
$\ldots$ & & & & & \\
\hline
\end{tabular}

Notes. The full table is available at the CDS. ${ }^{(a)}$ The flux is contaminated by extended emission near the source position.

selected on their expected submillimeter brightness considering their $70 \mu \mathrm{m}$ flux (brighter than $\sim 140 \mathrm{mJy}$ ). The details of these observations are discussed in Sect. 2.3.

The SEDs were further complemented with far-infrared fluxes from the Herschel Space Observatory (Pilbratt et al. 2010). The data reduction is discussed in Sect. 2.4.

\subsection{Submillimeter observations}

Observations of 32 of our targets were taken with the SABOCA and/or LABOCA instruments at the APEX telescope at the Chajnantor plateau in Chile. Observations were taken in service mode in 2012 and 2013 in ESO programs 089.C-0940, 090.C0820, and 091.C-0822 and Max Planck programs M0010_88 and M0003_90. The SABOCA instrument is a 39-channel bolometer array operating at $350 \mu \mathrm{m}$ (Siringo et al. 2010), and LABOCA is a 295-channel bolometer array at $870 \mu \mathrm{m}$ (Siringo et al. 2009). Imaging was performed in wobbler on-off mode. For a few sources, imaging was also performed in mapping mode (map size $\left.1.5^{\prime}\right)$ to check the pointing and to check for extended emission. One source (MP Mus, ID20) was observed with the new ArTeMiS camera in mapping during its commissioning phase, operating at $350 \mu \mathrm{m}$ (Revéret et al. 2014). Integration times were 5-40 min on source. The data were reduced using the CRUSH software (Kovács 2008) and (for the wobbler observations) verified using the BoA software (Schuller 2012). The results from both reduction techniques were found to agree within error bars and the CRUSH results are reported in Table 4. Flux calibration uncertainties (not included in Table 4) are typically $10 \%$ for LABOCA and $25-30 \%$ for SABOCA.

Observations of 41 of our targets were taken with the SCUBA-2 instrument at the JCMT telescope at Mauna Kea, Hawaii. Observations were taken in service mode in 2012 and 2013 in programs M12AN07, M12BN13, and M13AN01.

Max-Planck-Institut fur Radioastronomie, the European Southern Observatory, and the Onsala Space Observatory. 
Table 5. JCMT photometry at $850 \mu \mathrm{m}$ for our sample.

\begin{tabular}{ll|ll}
\hline \hline ID & $\begin{array}{l}F_{850 \mu \mathrm{m}} \\
(\mathrm{mJy})\end{array}$ & ID & $\begin{array}{l}F_{850 \mu \mathrm{m}} \\
(\mathrm{mJy})\end{array}$ \\
\hline 22 & $<31$ & 124 & $<19$ \\
23 & 153 & 127 & $<29$ \\
26 & $<30$ & 137 & $63 \pm 18$ \\
29 & $31 \pm 7$ & 154 & $<56$ \\
32 & $<47$ & 155 & $55 \pm 18$ \\
$\ldots$ & & & \\
\hline
\end{tabular}

Notes. The full table is available at the CDS. ${ }^{(a)}$ The flux is contaminated by extended emission near the source position.

The SCUBA-2 instrument is a 10000 pixel bolometer camera operating simultaneously at 450 and $850 \mu \mathrm{m}$ (Holland et al. 2013). Imaging was performed in the smallest possible map size (Daisy $3^{\prime}$ pattern). Observations were taken in grade 3-5 weather, which is generally insufficient for observing at $450 \mu \mathrm{m}$, so only the $850 \mu \mathrm{m}$ data are considered. Integration times were 5-50 min on source. The data were reduced using the default online pipeline. The resulting FITS images were inspected by eye for extended emission and fluxes and noise levels were derived. The noise levels were estimated by measuring the standard deviation in the map, after subtraction of point sources. The results are reported in Table 5. The flux calibration uncertainty (not included in Table 5) is typically 10\% for SCUBA-2.

\subsection{Herschel observations}

We searched the Herschel Science Archive for observations with the PACS broadband photometer (Poglitsch et al. 2010) at the coordinates of all targets in the sample. In photometry mode, PACS observes simultaneously at either 70 (PACS blue) and $160 \mu \mathrm{m}$ (PACS red) or 100 (PACS green) and $160 \mu \mathrm{m}$. Therefore, targets are recovered in either two or three of these wavelength bands. Only data products of reduction level higher than 2.0 were used, using the high pass filter.

Photometry of the PACS data was performed using the annularSkyAperturePhotometry task in the Herschel interactive processing environment (HIPE), version 12.1.0. This task derives background-corrected fluxes from point sources by comparing the flux inside a region centered on the point source and an annulus around it. We used the values for the aperture and annulus radii as used by Ribas et al. (2013). The background was estimated using the DAOPhot algorithm. Errors were estimated manually at several positions near the source position to avoid including nearby extended emission originating from clouds. The presence of nearby clouds is indicated in Table D.1. The flux calibration uncertainty (not included in Table D.1) is typically $5 \%$ for PACS photometry.

\subsection{Optical spectroscopy}

Stellar properties such as the spectral type must be determined to correct for extinction and deredden the SED flux points. The stellar luminosity is required to understand and interpret the SEDs properly. For about half of the targets in the sample, spectral types are available from the literature. The targets without known spectral type were observed with optical spectroscopy.

Optical spectra were taken for 90 targets, including reobservation of 24 targets for which the literature spectral type was still uncertain. We obtained low-resolution optical spectra with the Intermediate dispersion Spectrograph and Imaging System (ISIS) on the William Herschel Telescope ${ }^{3}$ from 4-8 August 2012. The D5700 dichroic splits the light at $5700 \AA$ into red and blue channels. The red emission then passes through the GG495 filter and is dispersed by the R158R grating to generate spectra from 5600-10000 $\AA$ at $R \sim 1200$. The blue emission is dispersed by the R300B grating to generate spectra from 3200-5800 $\AA$ at $R \sim 1800$.

The $R$ magnitudes ranged between 9 and 19 mag, requiring integration times of between 1 and $60 \mathrm{~min}$. The slit width was set each night depending on the seeing.

The spectra were reduced with custom written codes in IDL, including flat-field and cosmic ray corrections. The wavelength calibration was obtained from arc lamp spectra. An initial flux calibration was performed with observations of spectrophotometric standards G191 B2B, EG 274, G93-48, and LTT 6248, and was repeated several times each night (Oke 1990; Hamuy et al. 1992)

The spectral types of stars in our sample were estimated following the approximate method described by Herczeg \& Hillenbrand (2014), based on the spectral compilation by Pickles (1998) at early spectral types and Luhman (e.g., 2004) for late $K$ and $M$ dwarfs. For $K$ and $M$ stars with accretion, the spectral type estimates include a rough correction for veiling from the accretion continuum. The spectral types are estimated to be accurate to a few subclasses at spectral types earlier than $\mathrm{K} 5$ and $0.5-1$ subclass for late $\mathrm{K}$ and $\mathrm{M}$ stars. The $\mathrm{H} \alpha$ line equivalent width was calculated by fitting a Gaussian profile to the line.

\section{Results}

\subsection{Stellar parameters}

Spectral types as derived from our observations and taken from the literature are listed in Table A.2. The observations of previously characterized stars resulted generally in the same spectral types as derived before. Some of the WHT-ISIS targets did not show any lines and no spectral type could be determined. These targets are 19002346-3712242 (ID32), ISO-Oph43 (ID47), 18294721-0148301 (ID101), J182821.6+000016 (ID112), 18392594+0006382 (ID114), serp22 (124), 18401205+0029276 (ID125), Serp111 (ID131), J034219.3+314327 (ID164), J034345.17+320358.6 (ID202), and J162715.89-243843.2 (ID204), these SEDs were fit assuming a K7 star. For these stars, the temperature of $4060 \mathrm{~K}$ is indicated as (4060) in Table A.2. The targets J18272873-0406248 (ID68), 18304127-0242335 (ID80), 18291450-0220575 (ID84), 18314110-0128035 (ID104), 18385989-0008097 (ID110), $18374209+0016519$ (ID119), 18381580+0024218 (ID122), and $18381447+0035099$ (ID129) turned out to be giants, these SEDs were not analyzed further. There may be additional contamination in the sample by giants, especially in Serpens. For a handful of targets, the spectral type could not be determined to subtype accuracy. This paper presents new spectral types for 85 targets. For our final sample, spectral types are known for $\sim 85 \%$ of our targets. The uncertainty in the spectral type of a few subclasses results in less than 0.2 dex uncertainty in the bolometric luminosity, which is sufficient for our purposes of modeling the SED with a simple disk structure.

\footnotetext{
3 The William Herschel Telescope is operated on the island of La Palma by the Isaac Newton Group in the Spanish Observatorio del Roque de los Muchachos of the Instituto de Astrofísica de Canarias.
} 
Table 6. Comparison PACS photometry with previous estimates.

\begin{tabular}{llllllll}
\hline \hline \multirow{2}{*}{ ID } & \multicolumn{2}{c}{$F_{70 \mu \mathrm{m}}(\mathrm{Jy})$} & \multicolumn{2}{c}{$F_{100} \mu \mathrm{m}(\mathrm{Jy})$} & \multicolumn{2}{c}{$F_{160 \mu \mathrm{m}}(\mathrm{Jy})$} & Ref. \\
& This study & Previous & This study & Previous & This study & Previous & \\
\hline 4 & $<0.1$ & $<0.08$ & $<0.07$ & $<0.14$ & $<0.41$ & $<1.10$ & 1 \\
5 & $0.18 \pm 0.05$ & $0.15 \pm 0.02$ & $0.17 \pm 0.03$ & $0.17 \pm 0.04$ & $<0.33$ & $<1.07$ & 1 \\
6 & $3.11 \pm 0.31$ & $3.08 \pm 0.46$ & $2.90 \pm 0.29$ & $2.82 \pm 0.42$ & $2.15 \pm 0.25$ & $2.32 \pm 0.35$ & 1 \\
7 & $0.21 \pm 0.04$ & $<0.28$ & $0.21 \pm 0.03$ & $0.21 \pm 0.01$ & $<0.31$ & $<0.32$ & 2 \\
9 & $<0.65$ & $0.60 \pm 0.09$ & $<0.71$ & $0.77 \pm 0.12$ & $<1.06$ & $0.98 \pm 0.15$ & 1 \\
11 & $3.86 \pm 0.39$ & $3.88 \pm 0.58$ & $3.80 \pm 0.38$ & $3.63 \pm 0.54$ & $3.65 \pm 0.37$ & $3.86 \pm 0.58$ & 1 \\
12 & $0.44 \pm 0.05$ & $0.38 \pm 0.06$ & $0.40 \pm 0.05$ & $0.36 \pm 0.06$ & $<0.39$ & $0.20 \pm 0.03$ & 1 \\
13 & $<0.11$ & $<0.04$ & $0.14 \pm 0.03$ & $<0.07$ & $<0.55$ & $<0.85$ & 1 \\
14 & $0.69 \pm 0.08$ & $0.68 \pm 0.10$ & $0.55 \pm 0.06$ & $0.57 \pm 0.09$ & $0.41 \pm 0.07$ & $<0.30 \pm 0.05$ & 1 \\
15 & $1.58 \pm 0.16$ & $1.61 \pm 0.24$ & $2.31 \pm 0.23$ & $2.19 \pm 0.33$ & $2.80 \pm 0.28$ & $2.74 \pm 0.41$ & 1 \\
16 & $26.06 \pm 2.92$ & $25.91 \pm 3.88$ & $36.06 \pm 3.9$ & $32.32 \pm 4.85$ & $38.45 \pm 6.0$ & $27.3 \pm 4.10$ & 1 \\
17 & $0.21 \pm 0.05$ & $<0.25$ & $0.25 \pm 0.03$ & $0.23 \pm 0.01$ & $0.30 \pm 0.09$ & $0.28 \pm 0.05$ & 2 \\
24 & $0.17 \pm 0.04$ & $0.07 \pm 0.02$ & $0.11 \pm 0.03$ & $0.10 \pm 0.02$ & $<0.09$ & $<0.13$ & 3 \\
25 & $<0.34$ & $0.11 \pm 0.03$ & $<0.32$ & $0.16 \pm 0.04$ & $<0.38$ & $<0.23$ & 3 \\
26 & $<0.26$ & $0.10 \pm 0.02$ & $<0.12$ & $0.18 \pm 0.04$ & $<0.02$ & $<0.19$ & 3 \\
27 & $0.61 \pm 0.07$ & $0.51 \pm 0.13$ & $0.80 \pm 0.08$ & $0.68 \pm 0.17$ & $0.96 \pm 0.17$ & $0.72 \pm 0.18$ & 3 \\
179 & $1.23 \pm 0.13$ & $1.04 \pm 0.26$ & $1.41 \pm 0.14$ & $1.26 \pm 0.31$ & $1.69 \pm 0.19$ & $1.57 \pm 0.39$ & 3 \\
185 & $0.21 \pm 0.06$ & $0.17 \pm 0.04$ & $0.24 \pm 0.05$ & $0.23 \pm 0.06$ & $<0.91$ & $0.29 \pm 0.07$ & 3 \\
200 & $0.48 \pm 0.06$ & $0.36 \pm 0.09$ & $0.37 \pm 0.05$ & $0.37 \pm 0.09$ & $0.47 \pm 0.15$ & $0.26 \pm 0.07$ & 3 \\
\hline
\end{tabular}

References. 1) Ribas et al. (2013); 2) Olofsson et al. (2013); 3) Bustamante et al. (2015).

Spectral types are converted to the effective temperature $T_{\text {eff }}$ using the scales in Kenyon \& Hartmann (1995). The extinction $A_{V}$ and stellar luminosity $L_{*}$ (or stellar radius $R_{*}$, as $L_{*}=$ $4 \pi R_{*}^{2} \sigma T^{4}$ ) are fit simultaneously to the SEDs, assuming the distances listed at the bottom of Table A.1. Kurucz models of stellar photospheres (Castelli \& Kurucz 2004) are used as templates for the broadband emission. The 2MASS $J$-band and optical $V$ - and $R$-band fluxes are taken as reference to constrain the fit, assuming no excess in these bands, and assuming no significant veiling or variability through accretion or extinction (Cody et al. 2014; Stauffer et al. 2014). When both $V$ and $R$ were missing, the extinction was estimated adopting $A_{J}=1.53 \times E(J-K)$, where $E(J-K)$ is the observed color excess with respect to the expected photospheric color (Kenyon \& Hartmann 1995), depending on its spectral type. The extinction law is parametrized as a function of wavelength assuming $R_{V}=5.5$ (Indebetouw et al. 2005) and scaled to the visual extinction $A_{V}$. The resulting values are listed in Table A.2. Stellar masses are derived by interpolation of evolutionary models of Baraffe et al. (1998) in the position of the target on the HR diagram, although these are only approximations due to the uncertainties in the spectral type. For targets that could not be fit by the Baraffe models (which only include stars up to $1 M_{\odot}$ ), masses were derived using the evolutionary models by Siess et al. (2000). Since uncertainties in stellar age are large, they are not tabulated here. We note that for the Serpens targets an alternative distance of $250 \mathrm{pc}$ as used in previous work would often result in very high age estimates (>10 Myr), confirming that the $415 \mathrm{pc}$ used here is likely to be more accurate (which is also demonstrated in Oliveira et al. 2009, 2013). For ten targets no stellar mass could be derived, suggesting that their derived stellar properties are uncertain. Most of these are targets without known spectral type or late M stars.

The presence or absence of accretion can be assessed from the strength and shape of the emission of $\mathrm{H} \alpha$ and other optical lines (e.g., White \& Basri 2003; Natta et al. 2006). Although a proper treatment of the accretion requires simultaneous fitting of extinction, luminosity, and accretion through broadband spectroscopy (e.g., with X-shooter, Manara et al. 2014), as accretion also results in broadband UV/blue excess, the analysis in this study is limited to a simple designation of accretion by the width of the $\mathrm{H} \alpha$ line. We do not aim to quantify the accretion in terms of $M_{\odot} \mathrm{yr}^{-1}$ due to the large uncertainties when deriving accretion from the line width alone. Both the equivalent width $E W[\mathrm{H} \alpha]$ and the $\mathrm{H} \alpha 10 \%$ width have been used to distinguish between accretors and nonaccretors, where the $E W[\mathrm{H} \alpha]$ cutoff depends on the spectral type (White \& Basri 2003). Typically, a star is classified as an accretor if the $\mathrm{H} \alpha 10 \%$ width is $>300 \mathrm{~km} \mathrm{~s}^{-1}$ (Natta et al. 2004) or if $E W[\mathrm{H} \alpha]>3 \AA$ for an early-K star, $>10 \AA$ for a late-K star, and $>20 \AA$ for an M star. Since other studies often only list the $E W[\mathrm{H} \alpha]$ values, our accretion designation is largely based on those values.

In recent years, several YSOs have been analyzed with broadband high-resolution spectroscopy, including some of the targets in our sample (e.g., Alcalá et al. 2014; Manara et al. 2014). This accretion information is preferred to that derived from the equivalent width as this method is more reliable, those targets have been denoted explicitly in Table A.2. Accretion properties are known for $84 \%$ of our sample, and about $64 \%$ of these targets are accreting, the remaining targets show little or no signs of accretion.

\subsection{Long wavelength photometry}

The submillimeter photometry resulted in a total of 34 detections and 39 upper limits, listed in Table 4 and 5. In addition, we took (sub)millimeter photometry from the literature (see Table C.1). With 57 (sub)millimeter detections and 47 upper limits, about $50 \%$ of the targets in our sample have constraints at longer wavelengths.

Herschel-PACS surveys cover $92 \%$ of our targets. The derived fluxes and upper limits are listed in Table D.1 and images of the cut-out maps are given in Fig. D.1. For 152 targets, at least one of the three wavelengths results in a detection. For 18 targets, the emission is confused by cloud emission at all three wavelengths, for 27 only at 100 and $160 \mu \mathrm{m}$ and for 62 targets only at 
$160 \mu \mathrm{m}$. For 25 of the targets without cloud confusion, no flux is detected at any of the wavelengths.

The PACS $70 \mu \mathrm{m}$ fluxes and upper limits are consistent with the MIPS-2 fluxes and upper limits. The PACS sensitivity is sometimes shallower than the MIPS-2, resulting in a higher upper limit. For some targets, a more thorough data reduction of the PACS data was performed in other work (Ribas et al. 2013; Olofsson et al. 2013; Bustamante et al. 2015). In Table 6 the derived fluxes and upper limits are compared. Our values are similar within errors with previous estimates, confirming the validity of our data reduction.

\subsection{Disk parameters}

Millimeter fluxes can be used to obtain a rough estimate of the disk mass (gas+dust) assuming optically thin dust emission and a gas-to-dust ratio of 100 . Disk masses $M_{\text {disk,mm }}$ in our sample are calculated following the relations presented in Cieza et al. (2008) with standard assumptions and parameters

$M_{\text {disk }}=0.17\left(F_{1.3 \mathrm{~mm}}(\mathrm{mJy}) \times\left(\frac{d}{140 \mathrm{pc}}\right)^{2}\right) M_{\mathrm{Jup}}$

$M_{\text {disk }}=0.08\left(F_{0.85 \mathrm{~mm}}(\mathrm{mJy}) \times\left(\frac{d}{140 \mathrm{pc}}\right)^{2}\right) M_{\mathrm{Jup}}$,

with $F_{\lambda}$ the flux at wavelength $\lambda$ and $d$ the distance to the star. Using this relation, disk masses of our sample range between $<0.4$ and $168 M_{\text {Jup }}$, and an average disk mass of 14 Jupiter masses, similar to large millimeter studies of disks (e.g., Andrews \& Williams 2007a). However, these disk masses remain highly uncertain as the vertical structure, cavities, and stellar radiation field are not taken into account and the dust opacities and gas-to-dust ratio are uncertain.

Furthermore, we derive $L_{\text {disk }}$ for each target by integrating over all data points after subtraction of the fitted stellar photosphere. The ratio $L_{\text {disk }} / L_{*}$ is a measure of disk processing, as it traces the total amount of dust that is reprocessing stellar light. As disks become more tenuous, settle and eventually disappear, $L_{\mathrm{disk}}$ is expected to decline. The majority of the disks have $0.001<L_{\text {disk }} / L_{*}<0.4$, as expected for flared disks. Disks with $L_{\text {disk }} / L_{*}<10^{-3}$ are generally considered as debris disks (e.g., Wahhaj et al. 2010). On the other hand, targets with $L_{\text {disk }} / L_{*} \gg 1$ are either embedded Class I objects or edge-on disks, which are more difficult to analyze (Merín et al. 2010). ID178 has $L_{\text {disk }} / L_{*} \sim 17$ and is thus removed from the analyzed sample. Both $L_{\text {disk }} / L_{*}$ and $M_{\text {disk,mm }}$ are listed in Table A.3. The final sample consists of 184 targets for which the SEDs are analyzed.

\section{Modeling}

In order to determine the presence of a dust cavity and measure its size, the SEDs are modeled using the dust radiative transfer code RADMC-3D ${ }^{4}$ (Dullemond \& Dominik 2004). This code performs a Monte Carlo continuum radiative transfer calculation based on the input dust density profile and stellar photosphere, followed by ray tracing of the SED. The model has a large number of input parameters and we fixed as many of these parameters that are not important for our science goals as possible. The

\footnotetext{
4 www.ita.uni-heidelberg.de/ dullemond/software/ radmc-3d/
}

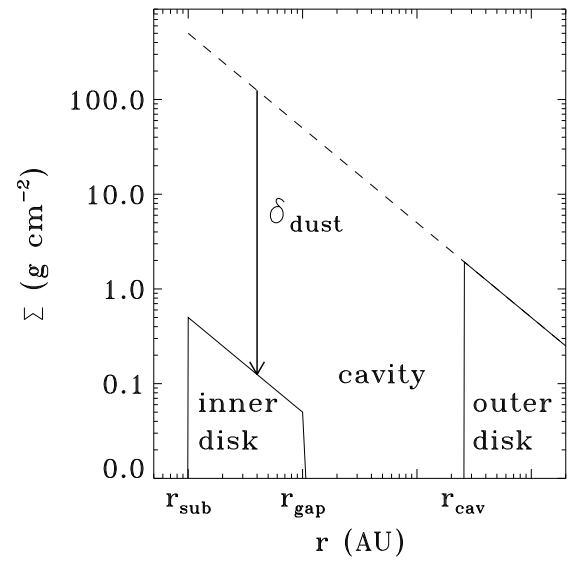

Fig. 1. Gas surface density profile used for modeling, assuming a gasto-dust ratio of 100 .

model assumes a passive disk that reprocesses the stellar radiation field.

The modeling procedure consists of two steps: first using a rough grid with a broad range of parameters, followed by a finer grid for the specific stellar type. The modeling was performed blindly, without taking any results from previous SED modeling or imaging studies, for an uniform approach for each disk in this sample. In Sect. 5 the derived parameters are compared with previously found results.

\subsection{Approach}

The disks are modeled using a large grid of models computed by RADMC-3D. The model assumes an axisymmetric gas surface density profile, following a radial power law

$\Sigma_{\mathrm{g}}(r)=\mathrm{GDR} \cdot \Sigma_{\mathrm{c}}\left(\frac{r}{r_{\mathrm{c}}}\right)^{-1}$,

where $r_{\mathrm{c}}$ is the characteristic radius and GDR is the gas-todust ratio set to 100 . The outer radius is set to $200 \mathrm{AU}$ and the inner radius to the sublimation radius $r_{\text {sub }}$ with $r_{\text {sub }}=$ $0.07\left(L_{*} / L_{\odot}\right)^{1 / 2}$, assuming a sublimation temperature of $1500 \mathrm{~K}$ (Dullemond et al. 2001). The characteristic radius $r_{\mathrm{c}}$ is set to 25 AU. The dust density inside the cavity is parametrized by setting the density equal to zero between $r_{\text {gap }}$ and $r_{\text {cav }}$. The inner disk (between $r_{\text {sub }}$ and $r_{\text {gap }}$ ) is set by varying $\delta_{\text {dust }}$ to fit the nearinfrared excess (see Fig. 1). The $r_{\text {gap }}$ is fixed to $1 \mathrm{AU}$ as it cannot be constrained well by the SED. A full disk without a cavity is simulated by setting $r_{\text {cav }}=r_{\text {gap }}$.

The stellar photosphere in the model is described by its temperature and stellar luminosity, which has been fit independently together with the extinction. The disk is assumed to be flared, so that the vertical structure of the disk is described by

$h(r)=h_{\mathrm{c}}\left(\frac{r}{r_{\mathrm{c}}}\right)^{\psi}$,

where $h_{\mathrm{c}}$ is the scale height at $r_{\mathrm{c}}$ and $\psi$ is the flaring angle, which are both varied to fit the near- and mid-infrared part of the SED. As the scale height is degenerate with the cavity radius, the flaring angle is taken as a conservative value of either $1 / 7$ or $2 / 7$, following Chiang \& Goldreich (1997). The derived cavity radius is thus likely a lower limit if the disk is flatter. With the inclusion of the Herschel fluxes, the scale height is better constrained than in previous SED modeling studies. Dust composition and settling 

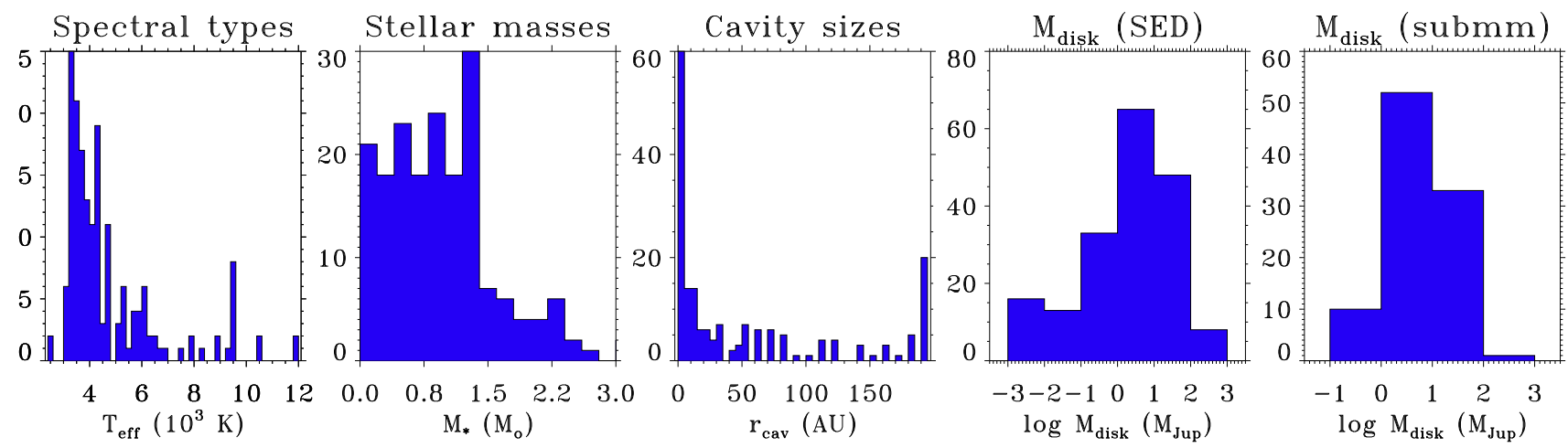

Fig. 2. Distribution of spectral types, stellar masses, cavity sizes, and disk masses (derived from SED fitting and from submillimeter flux) in this study. The disk mass is calculated assuming a gas-to-dust ratio of 100 .

is prescribed following Andrews et al. (2011) with a large and small dust grain population, where the large grains have a lower scale height than the small grains. The inclination of the disks is taken as a constant of $30^{\circ}$ and was not varied in the modeling, as only very high inclination angles (edge-on disks) result in a significant difference in the near-infrared emission (and in addition, obscuration of the star). With our color criteria, edge-on disks are not expected to be included (Merín et al. 2010) and also the computation of the stellar masses from the stellar luminosities implies that most of the targets are not edge-on (although higher inclinations than $30^{\circ}$ are still possible).

The five free parameters are thus $r_{\text {cav }}, \delta_{\text {dust }}, \Sigma_{\mathrm{c}}$ or disk mass, $h_{\mathrm{c}}$, and $\psi$, where $r_{\text {cav }}$ is the main parameter of interest. We note that $\Sigma_{\mathrm{c}}$ represents the dust surface density. The fitting was performed in two steps. First, a large grid of models with a broad range of disk parameters and a limited number of stellar parameters was fit to each SED. Second, each SED was fine-tuned individually, using the exact stellar photosphere and starting from the best fit from the broad grid. The disk grids per object have a large range of cavity radii (our main parameter of interest), in combination with a small range of scale heights and disk masses. Although this approach is rather simple, results of SED modeling are known to be highly uncertain, especially for those targets without known spectral type, and the fitting results should only be considered as a first approximation of the structure. More detailed analysis and imaging data are required to fit individual targets more accurately.

In the fitting procedure, a $\chi^{2}$ minimization was performed between the dereddened SED data points and model SEDs. In the grid fitting, the data points were weighted by their excess above the stellar photosphere at each wavelength, fluxes at longer wavelengths got a larger weight than those in the optical and near-infrared since the stellar photosphere is largely known from the extinction fitting. Uncertainties on the cavity radii are given in Table A.3, based on fits with up to $10 \%$ variation in $\chi^{2}$.

\subsection{Results}

Each SED can be fit to a disk model with the majority of disks containing a cavity. Table A.3 presents the results of the fitting procedure. Figure 2 presents the distribution of hole sizes and disk masses (assuming a gas-to-dust ratio of 100) of the full sample, showing a broad distribution of both parameters. The disk masses obtained from the fit generally agree within a factor of 2-3 with the mass estimate from the millimeter flux. During the fit procedure it became clear that certain disks have really large cavities (>100 AU) but very low scale heights that cannot be reproduced well by our flared models. These disks are likely debris disks, as is also suggested by their low $L_{\text {disk }} / L_{*}$ values. Recently, a sample of similar young WTTS disks were found to be gas-poor debris disks through ALMA observations (Hardy et al. 2015).

Figures B.1 to B.6 present the SEDs with the best-fitting models overlaid. The SEDs are grouped into the following five different classifications:

- NH: disks without holes $\left(r_{\mathrm{cav}}=1 \mathrm{AU}\right)$;

- LS: low-mass disks with small holes

$\left(r_{\text {cav }}<10 \mathrm{AU}, M_{\text {disk }}<5 M_{\text {Jup }}\right)$;

- LL: low-mass disks with large holes

$\left(r_{\text {cav }}>10 \mathrm{AU}, M_{\text {disk }}<5 M_{\text {Jup }}, h_{\mathrm{c}}>0.01\right)$;

- MS: massive disks with small holes $\left(r_{\text {cav }}<10 \mathrm{AU}, M_{\text {disk }}>5 M_{\text {Jup }}\right)$;

- ML: massive disks with large holes $\left(r_{\text {cav }}>10 \mathrm{AU}, M_{\text {disk }}>5 M_{\text {Jup }}\right)$;

- DD: low-mass disks with large holes and very low scale heights

$\left(r_{\text {cav }}>100 \mathrm{AU}, M_{\text {disk }}<5 M_{\text {Jup }}, h_{\mathrm{c}} \sim 0.01\right)$.

For the disks classified as NH (no hole), we have excluded the targets that could be fit with a cavity $>1$ AU within the $10 \% \chi^{2}$ limit.

A large fraction of the disks $(\sim 23 \%)$ falls in the ML category of large holes in massive disks. It turns out that several of these disks are indeed the famous, bright disks with large inner holes known from imaging surveys (Andrews et al. 2011; Williams \& Cieza 2011), confirming the strength of our SED modeling, even if rather simple. The new targets in the ML, MS, and some in the LL groups are promising disks for follow-up observations with ALMA. Excluding the DD and NH disks, a total of 133 targets ( $72 \%$ of our analyzed sample) can be labeled as disks with cavities, i.e., transition disks. More than half of these ( $~ 70$ targets) are new transition disks, about 40 had been imaged or modeled before and another 20 had been recognized as a possible transition disk. Of the new transition disks, two-thirds have a known spectral type.

\section{Discussion}

The SED modeling has confirmed the presence of cavities in a large sample of transition disks. At least $72 \%$ of the sample could be modeled as a disk with a cavity, including about 110 new transition disks that had not been identified as transition disks before. 
Table 7. Comparison cavity radii with literature values.

\begin{tabular}{|c|c|c|c|c|c|c|c|c|c|c|c|}
\hline ID & Name & $r_{\text {cav }}^{\text {here }}$ & $r_{\text {cav }}^{\text {lit }}$ & Type $^{a}$ & Ref. & ID & Name & $r_{\text {cav }}^{\text {here }}$ & $r_{\text {cav }}^{\text {lit }}$ & Type $^{a}$ & Ref. \\
\hline 1 & TCha & $140_{-10}^{+10}$ & 19 & I & 1 & 51 & IRS48 & $120_{-10}^{+10}$ & 60 & I & 13 \\
\hline 3 & RECX5 & $10_{-2}^{+2^{-10}}$ & 33 & M & 2 & 52 & DoAr44 & $80_{-20}^{+10}$ & 30 & I & 10 \\
\hline 4 & CHXR22E & $45_{-5}^{+15}$ & 7 & M & 3 & 54 & SR21 & $60_{-15}^{+20}$ & 36 & I & 8 \\
\hline 6 & CSCha & $60^{-5}+10$ & 38 & M & 4 & 60 & oph62 & $2^{+2}$ & 3 & $\mathrm{M}$ & 6 \\
\hline 9 & T54 & $120_{-10}^{+10}$ & 37 & M & 3 & 64 & $\mathrm{~J} 160421$ & $70_{-30}^{+20}$ & 80 & I & 14 \\
\hline 10 & $\mathrm{~T} 21$ & $190_{-10}^{-10}$ & 146 & M & 3 & 120 & J182911 & $10^{-30}$ & 8 & $\mathrm{M}$ & 6 \\
\hline 11 & SZCha & $30_{-10}^{+10}$ & 29 & M & 3 & 127 & Serp127 & $80_{-10}^{+20}$ & 25 & M & 6 \\
\hline 12 & $\mathrm{~T} 35$ & $15_{-5}^{-10}$ & 15 & M & 3 & 128 & J182935 & $25_{-23}^{+10}$ & 7 & M & 6 \\
\hline 14 & T56 & $10_{-4}^{+4}$ & 18 & M & 3 & 135 & DMTau & $4_{-2}^{+23}$ & 18 & I & 10 \\
\hline 15 & CRCha & $1^{+1^{-4}}$ & 10 & M & 4 & 136 & UXTauA & $50_{-10}^{+2}$ & 25 & I & 10 \\
\hline 18 & $\mathrm{~T} 25$ & $30_{-5}^{+5}$ & 8 & M & 3 & 142 & MWC758 & $25^{-15}$ & 73 & I & 10 \\
\hline 21 & HD142527 & $110^{-5}+10$ & 100 & I & 5 & 148 & IPTau & $100^{+5}+10$ & 2 & $\mathrm{M}$ & 4 \\
\hline 24 & Lup60 & $16^{+2}$ & 3 & $\mathrm{M}$ & 6 & 153 & RYTau & $2^{+2^{-30}}$ & $26^{b}$ & I & 15 \\
\hline 27 & Sz91 & $120_{-20}^{+40}$ & 97 & I & 7 & 159 & ABAur & $1^{+4}$ & 115 & I & 16 \\
\hline 29 & Sz84 & $70_{-10}^{+40}$ & 55 & M & 6 & 161 & ASR118 & $2^{+4}$ & 1 & $\mathrm{M}$ & 6 \\
\hline 33 & HD135344 & $80_{-10}^{+10}$ & 46 & I & 8 & 165 & J034227 & $8_{-4}^{+4}$ & 5 & M & 6 \\
\hline 35 & Sz76 & $2^{+2^{-10}}$ & 1 & M & 9 & 168 & J034434 & $5^{-4}+20$ & 3 & $\mathrm{M}$ & 6 \\
\hline 38 & RXJ1615 & $10^{+10}$ & 30 & I & 10 & 169 & IC348LRL190 & $2^{-3}$ & 5 & M & 6 \\
\hline 39 & V4046Sgr & $16_{-6}^{+2}$ & 29 & I & 11 & 173 & LkH-alpha330 & $120_{-20}^{+10}$ & 68 & I & 10 \\
\hline 45 & SR24S & $50^{-6}+40$ & 30 & I & 10 & 309 & TWHyа & $10^{+2}$ & 4 & $\mathrm{M}$ & 17 \\
\hline 46 & RXJ1633 & $20^{-20}$ & 27 & I & 12 & 325 & LkCa15 & $80^{-40}$ & 50 & I & 10 \\
\hline 48 & WSB60 & $8^{+6^{-5}}$ & 15 & I & 10 & 326 & CoKu-Tau-4 & $6^{+2^{-3}}$ & 10 & $\mathrm{M}$ & 18 \\
\hline 50 & $\mathrm{~J} 162245$ & $2^{-6}+2$ & 1 & $\mathrm{M}$ & 6 & 329 & GMAur & $30^{-2}+10$ & 20 & $\mathrm{I}$ & 10 \\
\hline
\end{tabular}

Notes. ${ }^{(a)} \mathrm{M}=$ derived from SED modeling, $\mathrm{I}=$ derived from millimeter imaging. ${ }^{(b)}$ This value is not a real cavity size, but a transition radius: the disk was fit with a surface density profile that radially increases and decreases, peaking at $26 \mathrm{AU}$.

References. (1) Huélamo et al. (2015); (2) Bouwman et al. (2010); (3) Kim et al. (2009); (4) Espaillat et al. (2011); (5) Fukagawa et al. (2013); (6) Merín et al. (2010); (7) Canovas et al. (2015); (8) Brown et al. (2009); (9) Padgett et al. (2006); (10) Andrews et al. (2011); (11) Rosenfeld et al. (2013); (12) Cieza et al. (2012a); (13) van der Marel et al. (2013); (14) Mathews et al. (2012); (15) Isella et al. (2010a); (16) Piétu et al. (2005); (17) Andrews et al. (2012); (18) D’Alessio et al. (2005).

\subsection{Comparison of cavity radii with literature values}

In order to quantify the quality of our models, the fit results for the cavity size are compared with values from the literature from both SED modeling and resolved millimeter imaging in Table 7 and Fig. 3. The cavity radii generally agree well within a factor of two with previously derived parameters. The similarity to the imaging results is especially encouraging, this implies that a large number of our new targets are suitable for resolved imaging. Exceptions for the imaging targets are T Cha, RY Tau, and $\mathrm{AB}$ Aur. For $\mathrm{T}$ Cha the overestimate of the cavity size could be caused by the assumed low inclination in our models, imaging has shown that the inclination is in reality $\sim 67^{\circ}$ so close to edge-on (Huélamo et al. 2015), affecting the near-infrared emission from the inner disk. Also, their flaring angle is lower than ours. For RY Tau, the cavity radius found by imaging is not defined in the same way as our cavity radius, the given radius is the peak of the mm dust surface density, assuming a surface density that first increases and then decreases with radius (Isella et al. 2010a). Therefore, the values cannot be compared directly. For AB Aur, only a very small hole of at most 2 AU can be fit with our models, while millimeter imaging has revealed a large cavity of $115 \mathrm{AU}$ at $1.4 \mathrm{~mm}$ with a complex, possibly spiral-arm structure (Piétu et al. 2005; Tang et al. 2012). As the AB Aur disk is still embedded in a envelope, the mid-infrared emission is likely confused by cloud emission, which can explain this discrepancy between the SED and millimeter image. The comparison with

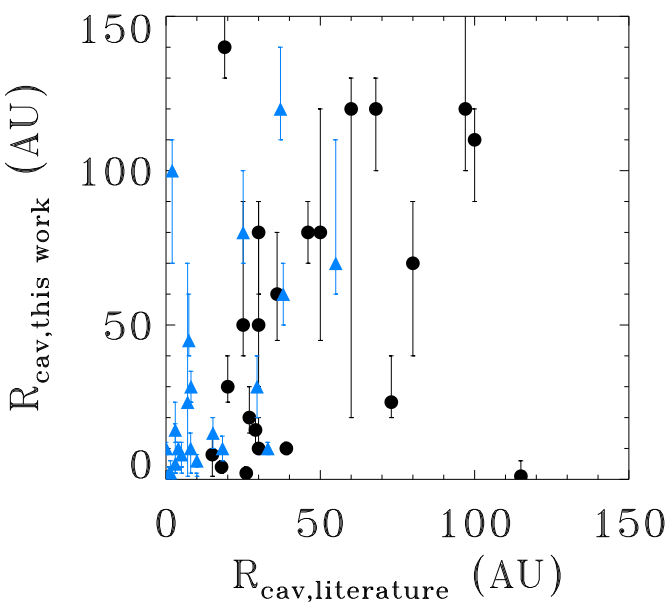

Fig. 3. Comparison derived cavity radii with literature values, based on Table 7. The black circles indicate the literature results from imaging, the blue diamonds from modeling.

SED modeling shows large discrepancies for CHXR22E, T54, CR Cha, and IP Tau. These targets were not modeled with a full radiative transfer code, they were modeled with a parametrized temperature profile and optically thin dust emission inside the cavity rather than an inner disk (Kim et al. 2009; Espaillat et al. 2011), so the results cannot be compared directly. 
Table 8. Multiplicity properties.

\begin{tabular}{|c|c|c|c|c|c|c|c|c|c|c|c|}
\hline ID & Name & $\begin{array}{l}\text { Sep. } \\
(\operatorname{arcsec})\end{array}$ & $\begin{array}{l}\text { Sep. } \\
(\mathrm{AU})\end{array}$ & $\begin{array}{l}r_{\mathrm{cav}} \\
(\mathrm{AU})\end{array}$ & Ref. & ID & Name & $\begin{array}{l}\text { Sep. } \\
(\operatorname{arcsec})\end{array}$ & $\begin{array}{l}\text { Sep. } \\
(\mathrm{AU})\end{array}$ & $\begin{array}{l}r_{\mathrm{cav}} \\
(\mathrm{AU})\end{array}$ & Ref. \\
\hline 1 & TCha & 0.062 & 6.7 & $140_{-10}^{+10}$ & 1 & 60 & oph62 & $<0.1$ & $<12$ & $2^{+2}$ & 9 \\
\hline 4 & CHXR22E & $<0.1$ & $<16$ & $45_{-5}^{+15}$ & 2 & 62 & J162218.5-232148 & $<0.1$ & $<12$ & 1 & 9 \\
\hline 5 & ISO52 & $<0.1$ & $<16$ & $30_{-18}^{+50}$ & 2 & 63 & DoAr28 & $<0.13$ & $<16$ & $20_{-5}^{+5}$ & 10 \\
\hline 6 & CSCha & - & 4 & $60_{-10}^{+18}$ & 3 & 64 & $\mathrm{~J} 160421.7-213028$ & $<0.01$ & $<1.5$ & $70_{-30}^{+20}$ & 11 \\
\hline 7 & $11094742-7726290$ & $<0.75$ & $<120$ & $8_{-2}^{+2^{-10}}$ & 4 & 134 & RXJ0432.8+1735 & $<0.13$ & $<18$ & $190_{-10}^{-30}$ & 12 \\
\hline 9 & T54 & 0.25 & 40 & $120_{-10}^{+20}$ & 2 & 135 & DMTau & $>0.03$ & $>4$ & $4_{-2}^{+2}$ & 13 \\
\hline 10 & $\mathrm{~T} 21$ & 0.14 & 22 & $190_{-10}^{-10}$ & 2 & 136 & UXTauA & $>0.03$ & $>4$ & $50_{-10}^{+240}$ & 13 \\
\hline 11 & SZCha & $<0.07$ & $<11$ & $30_{-10}^{+10}$ & 2 & 140 & $043649.1+241258$ & $<0.1$ & $<14$ & $190_{-10}^{-10}$ & 14 \\
\hline 12 & $\mathrm{~T} 35$ & $<0.07$ & $<11$ & $15_{-5}^{+10}$ & 2 & 149 & V892Tau & 0.06 & 8 & $10_{-8}^{+8}$ & 15 \\
\hline 14 & T56 & $<0.07$ & $<11$ & $10_{-4}^{+4}$ & 2 & 150 & V410X-ray6 & $<0.1$ & $<14$ & $15_{-5}^{+5}$ & 14 \\
\hline 15 & CRCha & $<0.08$ & $<13$ & $1^{+1^{-4}}$ & 2 & 152 & V819Tau & $<0.1$ & $<14$ & $150_{-20}^{+20}$ & 14 \\
\hline 16 & WWCha & $<0.12$ & $<19$ & $50_{-49}^{+30}$ & 2 & 153 & RYTau & $>0.03$ & $>4$ & $2^{+2}$ & 13 \\
\hline 17 & $11062554-7633418$ & $<0.75$ & $<120$ & $15_{-5}^{+15}$ & 4 & 162 & MBO22 & $<0.1$ & $<25$ & $2^{+2}$ & 14 \\
\hline 18 & $\mathrm{~T} 25$ & $<0.08$ & $<13$ & $30_{-5}^{+5}$ & 2 & 172 & IC348-67 & $<0.1$ & $<25$ & $2^{+1}$ & 14 \\
\hline 21 & HD142527 & 0.088 & 13 & $110_{-20}^{+10}$ & 5 & 174 & $\mathrm{~J} 04300424+3522238$ & $<0.1$ & $<45$ & $18_{-6}^{+10}$ & 14 \\
\hline 23 & Sz111 & $<0.7$ & $<140$ & $60_{-10}^{+10}$ & 6 & 175 & $\mathrm{~J} 04303235+3536133$ & 0.83 & 116 & $4_{-2}^{+8^{-6}}$ & 14 \\
\hline 24 & Lup60 & $<0.1$ & $<20$ & $16_{-4}^{+20}$ & 7 & 177 & $\mathrm{~J} 04304004+3542101$ & 1.2 & 168 & $25_{-10}^{+10}$ & 14 \\
\hline 25 & J160830.3-390611 & $<0.8$ & $<160$ & $4_{-2}^{+4^{-4}}$ & 6 & 179 & $\mathrm{~J} 160044.5-415531$ & $<0.1$ & $<15$ & $1^{+59^{-10}}$ & 7 \\
\hline 27 & Sz91 & $<0.1$ & $<20$ & $120_{-20}^{+30}$ & 7 & 180 & J190058.1-364505 & 0.5 & 72 & $14_{-4}^{+4}$ & 7 \\
\hline 28 & J160855.5-390234 & $<0.1$ & $<20$ & $2^{+2}$ & 7 & 181 & $03445614+3209152$ & $<0.1$ & $<25$ & $6_{-5}^{+12}$ & 14 \\
\hline 33 & HD135344 & $<0.1$ & $<14$ & $80_{-10}^{+10}$ & 8 & 182 & $03442156+3215098$ & $<0.1$ & $<25$ & $2^{-3}+23$ & 14 \\
\hline 40 & J163154.7-250324 & $<0.1$ & $<12$ & 1 & 9 & 183 & $03442257+3201536$ & $<0.1$ & $<25$ & $4_{-2}^{+1}$ & 14 \\
\hline 41 & J163205.5-250236 & $<0.1$ & $<12$ & 1 & 9 & 184 & $04330422+2921499$ & $<0.1$ & $<25$ & $160_{-10}^{+20}$ & 14 \\
\hline 43 & J163023.4-245416 & $<0.13$ & $<16$ & $45_{-20}^{+5}$ & 10 & 191 & $042921.6+270125$ & 0.22 & 30 & $2^{+2^{-10}}$ & 16 \\
\hline 44 & WSB63 & $<0.1$ & $<12$ & $4_{-2}^{+20}$ & 9 & 200 & J160710.08-391103.5 & $<0.06$ & $<12$ & 1 & 6 \\
\hline 46 & RXJ1633.9-2442 & $<0.1$ & $<12$ & $20_{-5}^{+2}$ & 9 & 309 & TWHya & $<0.1$ & $<5$ & $10_{-2}^{+2}$ & 8 \\
\hline 47 & ISO-Oph43 & $<0.13$ & $<16$ & $1^{+1^{-3}}$ & 10 & 318 & DoAr21 & - & 1.5 & $70_{-10}^{+30}$ & 17 \\
\hline 48 & WSB60 & $<0.1$ & $<12$ & $8_{-7}^{+6}$ & 8 & 319 & J162740.3-242204 & 0.638 & 80 & $1^{+17}$ & 10 \\
\hline 49 & J163115.7-243402 & 0.33 & 41 & $20_{-10}^{+130}$ & 9 & 325 & LkCa15 & $>0.03$ & $>4$ & $80_{-35}^{+40}$ & 13 \\
\hline 50 & J162245.4-243124 & 0.54 & 68 & $2^{+2^{-10}}$ & 9 & 326 & CoKu-Tau-4 & 0.053 & 8 & $6_{-2}^{+2^{-3}}$ & 18 \\
\hline 58 & J162648.6-235634 & $<0.13$ & $<16$ & 1 & 10 & 329 & GMAur & $>0.03$ & $>4$ & $30_{-5}^{+10}$ & 13 \\
\hline 59 & J162802.6-235504 & $<0.1$ & $<12$ & $2^{+2}$ & 9 & & & & & & \\
\hline
\end{tabular}

References. (1) Huélamo et al. (2011); (2) Lafrenière et al. (2008); (3) Guenther et al. (2007); (4) Comerón (2012); (5) Biller et al. (2012); (6) Ghez et al. (1997); (7) Romero et al. (2012); (8) Vicente et al. (2011); (9) Cieza et al. (2010); (10) Ratzka et al. (2005); (11) Kraus et al. (2008); (12) Kohler \& Leinert (1998); (13) Pott et al. (2010); (14) Cieza et al. (2012b); (15) Leinert et al. (1997); (16) Biller et al. (2011); (17) Loinard et al. (2008); (18) Ireland \& Kraus (2008).

\subsection{Binaries}

Some transition disks can be explained as circumbinary disks due to the dynamical interaction between the disk and a stellar companion. The cavity size is expected to be $\sim 2$ times as large as the binary separation (Artymowicz \& Lubow 1996). The fraction of the transition disks in our sample for which binarity has been studied is limited, but for those targets where spatially resolved information is available from the literature (either detections or upper limits), the properties are listed in Table 8, together with the cavity sizes found in this study.

Although the cavity can indeed be explained by a binary companion for a handful of targets, the limits are not sufficient to exclude circumbinary disks for the bulk of the disks. Previous binary studies of transition disks also revealed that most of the sharp cavities are not due to binary systems (Pott et al. 2010; Vicente et al. 2011).

\subsection{Accretion}

By combining the outcome of the SED modeling with our information on accretion, the possibility that photoevaporation is the origin of the cavities can be checked. According to photoevaporation models (e.g., Alexander et al. 2006), UV photons from the star heat and ionize the gas in the disk, beyond a critical radius, the thermal velocity of the ionized gas exceeds its escape velocity and the material is dissipated as a wind. During the lifetime of the disk, the accretion rate is expected to gradually decrease, and when the rate drops below the photoevaporation rate, the outer disk can no longer resupply the inner disk with material and an inner hole is formed. This process is called photoevaporative clearing, and transition disks created by this mechanism are expected to have no or very low accretion, although only disks with small inner cavities can be explained by this mechanism (Owen 2016). Clearing of a gap by a planet and photoevaporation could also happen simultaneously (e.g., Rosotti et al. 2013), making the distinction not purely measurable by accretion alone. 

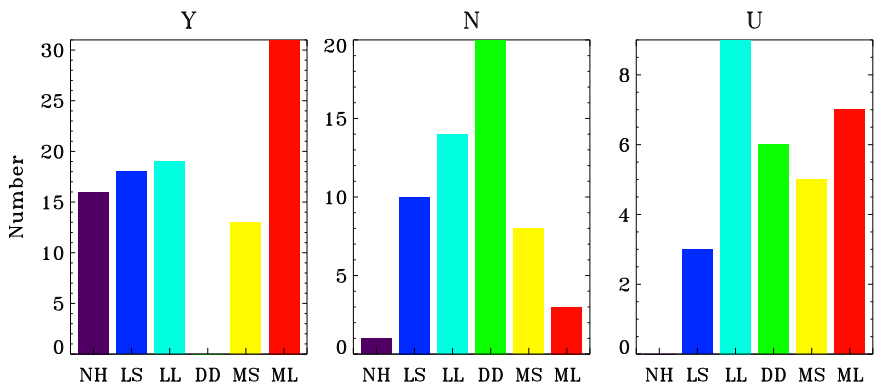

Fig. 4. Comparison of accretion properties from Table A.2 with disk hole parameters: $\mathrm{Y}$ means accreting, $\mathrm{N}$ means nonaccreting, and U means unknown.
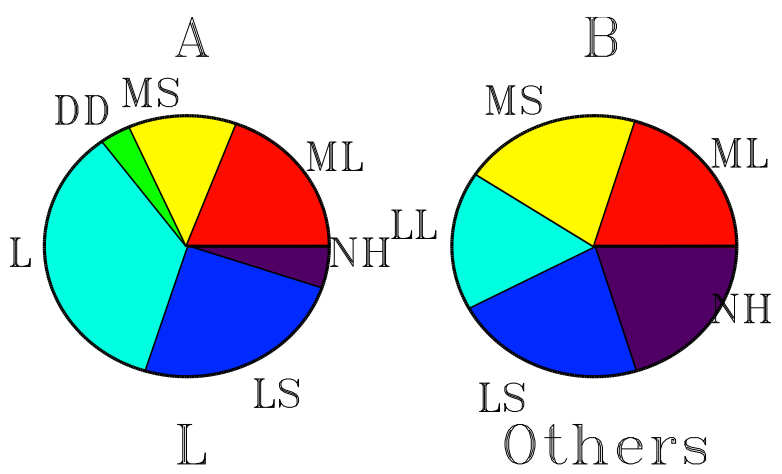

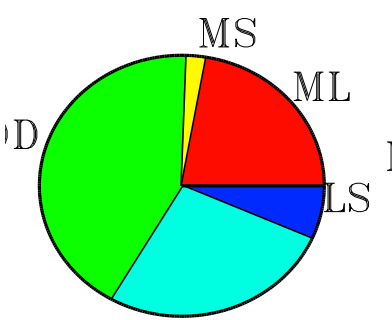

LL
Others

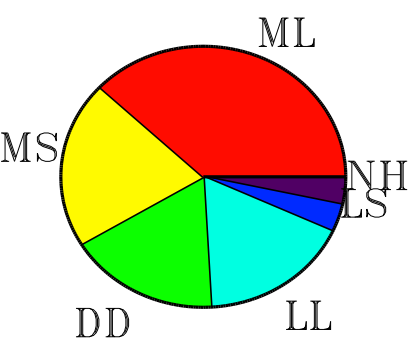

DD
LL
Fig. 5. Evaluation of the color criteria (Region A, B and L colors) vs. the outcome of the SED modeling.

Figure 4 shows the number of objects in each class that are accreting/nonaccreting. The accretors are dominated by disks without holes and massive disks with large holes, which are likely transition disks with a cavity due to clearing by a companion. The nonaccretors are dominated by the low scale height, lowmass disks (DD), confirming that they are likely debris disks. The nonaccreting, low-mass disks are possibly disks where the hole is caused by photoevaporative clearing, which is consistent with the so-called mm-faint disks (Owen \& Clarke 2012; Owen 2016) in contrast with the mm-bright transitional disks. On the other hand, there are several low-mass accreting disks as well, so there is no general trend for the low-mass disks. The nonaccreting disk without a hole (J034520.5+320634, ID171) is an outlier, but the equivalent width of this target is on the edge of accreting/nonaccreting, probably because of the ubiquitous variable accretion (Mendigutía et al. 2012; Venuti et al. 2014), and should thus have been classified as an accretor.

\subsection{Evaluation of color criteria}

Considering the high success rate of new transition disks found in the sample in this study, it is now possible to re-evaluate the criteria used to select the targets. Figure 5 presents the resulting

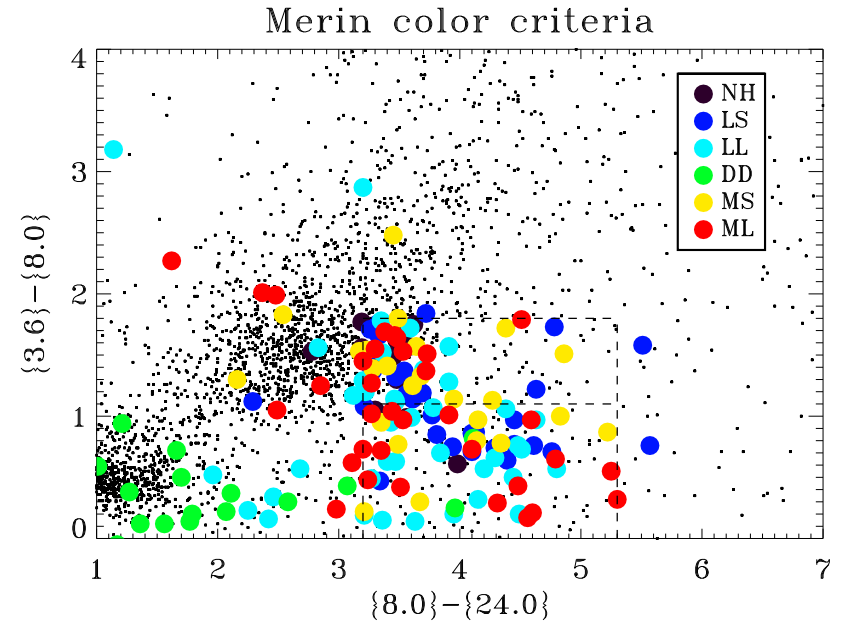

Fig. 6. Evaluation of the color criteria (A and B) vs. the outcome of the SED modeling. The filled symbols are the targets analyzed in this study, the dots are all YSOs in the c2d, GB, and Taurus catalogs. The dashed lines indicate Region B (top) and Region A (bottom) criteria.
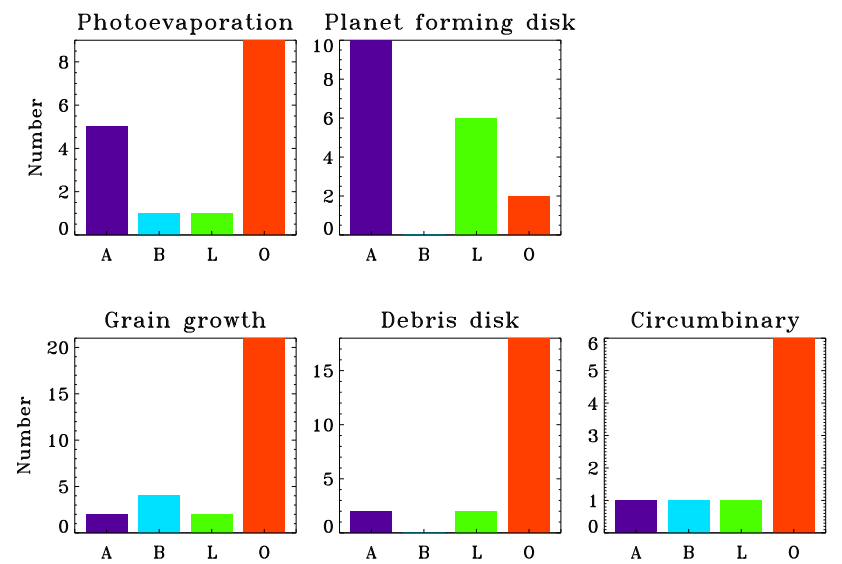

Fig. 7. Comparison of the categorization by the Cieza et al. studies with the color criteria used in this study applied to the 92 targets in the Cieza sample.

classifications from our SED modeling for each of the four color selection criteria.

From Fig. 5 it is clear that the disks without holes are most dominant in the B criteria, but overall at a low fraction $(<10 \%)$. The L criteria are particularly biased toward the low scale height disks and low-mass disks with large holes. Massive disks with large holes are found in all colors. Evaluating the M10 criteria directly in the color-color plot, the DD disks fall outside the A and B range. A small amount of disks with holes falls outside the range of the A, B criteria (these are targets from the literature), and generally have a shallower 8-24 $\mu \mathrm{m}$ slope.

\subsection{Comparison with Cieza survey}

A previous large survey of transition disk candidates was performed by Cieza et al. (2010, 2012b) and Romero et al. (2012), who selected a sample of candidates using their own color criteria. Rather than SED modeling, they apply criteria based on a range of observables (disk mass, $L_{\text {disk }} / L_{*}$, accretion, multiplicity, infrared spectral slope $\alpha_{\text {excess }}$, and the wavelength where the disk emission starts to dominate, $\lambda_{\text {turnoff }}$ ) to determine the origin 


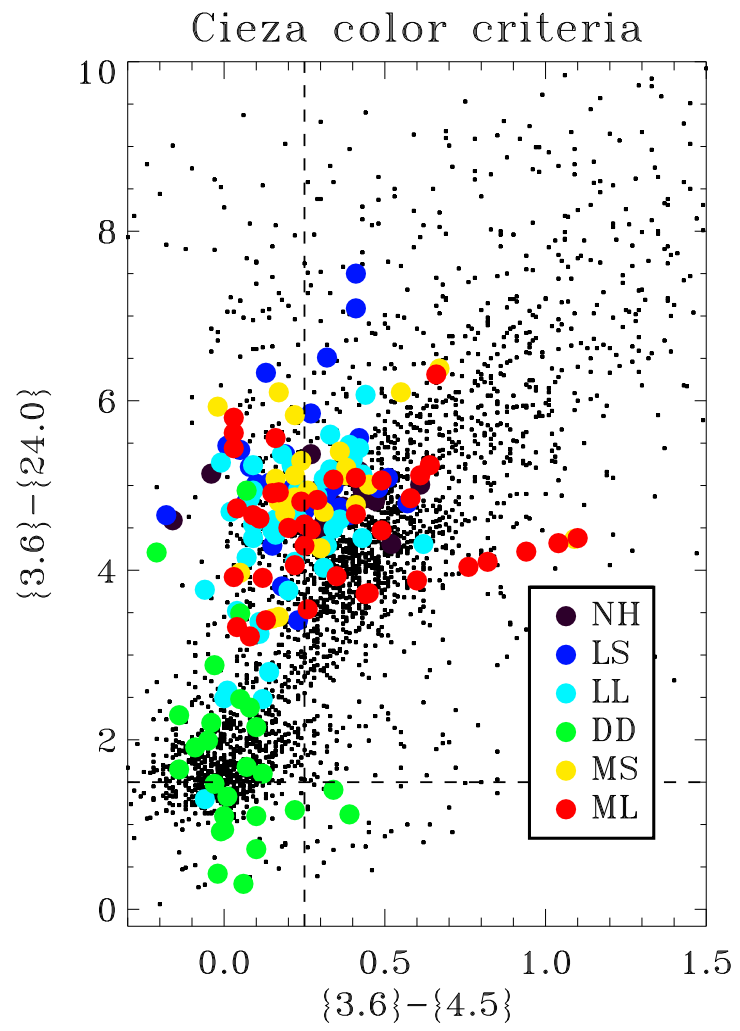

Fig. 8. Evaluation of the initial color criteria by the Cieza survey vs. the outcome of our SED modeling. The filled symbols are the targets analyzed in our study, and the dots are all YSOs in the c2d, GB, and Taurus catalogs. The dashed lines indcate the regions: the upper left quartile are the transition disks according to Cieza, and the lower left quartile are diskless stars.

of the dust deficit in their disks: circumbinary disk, photoevaporative clearing, debris disk, grain growth, or planetary clearing.

Of particular interest are their planet-forming disks, which are massive, accreting disks with sharp cavities $\left(\alpha_{\text {excess }}>0\right)$. Their final target list is compared with our sample and the colors used for our color criteria have been derived for all their targets. We note that 27 of their 92 targets are already present in our sample, either selected by the color criteria or by their classification in the literature. Figure 7 shows how their categorization overlaps with our color criteria. Our A and L color criteria are clearly favored in the planet forming disks, while many of the less interesting disks from our perspective (grain growth, debris disks) fall in category, "O", outside our color criteria. Several of the photoevaporation disks also follow the A criteria. This comparison confirms that our color criteria are good at selecting disks with sharp inner cavities.

Figure 8 shows the inital color selection of Cieza et al. $([3.6]-[4.5]<0.25,[3.6]-[24.0]>1.5)$ in comparison with the outcome of our classification. This Figure shows that only $50 \%$ of our disks with holes fall within these criteria. One of the reasons is that Cieza et al. have stricter constraints for their near-infrared emission, which excludes the transition disks with strong near-infrared excess (indicating an inner disk). The DD targets fall in the same quartile as the diskless stars, as expected.

Finally, Fig. 9 shows how the classification of Cieza et al. compares with our classification for our targets. We included all of our targets in the comparison, computing the values of $\alpha_{\text {excess }}$ and $\lambda_{\text {turnoff }}$ ourselves in order to put them in the Cieza classification. Planet forming disks fall mostly within the class
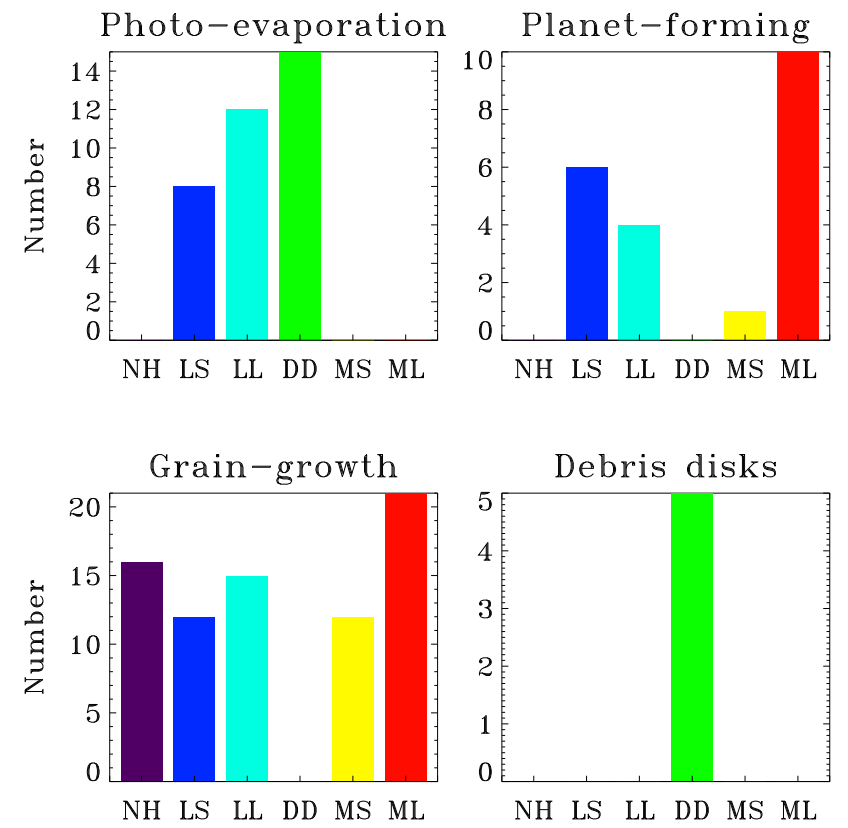

Fig. 9. Comparison of the categorization by the Cieza et al. studies with the classification of this study for our targets.

of massive disks with large cavities, while photoevaporation and debris disks are mostly consistent with low-mass disks. Neither of these findings is a surprise, considering the categorization of Cieza et al. (2010). Disks without holes all fall within the category of grain-growth disks. On the other hand, several other disks in the grain-growth category could be fit with a disk including a cavity. Circumbinarity is not well constrained for most of our sample and therefore not included.

\subsection{Evolution}

The $L_{\text {disk }} / L_{*}$ ratio is generally taken as a measure of disk evolution. For the targets in this study, there is a hint of a trend with larger cavity radii and generally more nonaccretors for lower $L_{\text {disk }} / L_{*}$ (Fig. 10a). This implies a general growth of cavity sizes with time while accretion decreases.

A relation between disk mass and cavity radius has been noted in previous studies and interpreted as a gravitational process, where larger disk masses produce more massive planets, clearing larger cavities (Merín et al. 2010). This study shows no trend between disk mass and cavity size. However, accretors are generally more massive than nonaccretors (Fig. 10b).

Both trends are susceptible, owing to the uncertainties in $r_{\text {cav }}$, and biased because of the presence of many low-mass disks with large cavities in our sample, these are more likely to be debris disks than transition disks. When the DD disks are removed from the sample, there is barely a visible trend.

\subsection{Cavity radii and exoplanets}

If the origin of the dust cavity radii lies in forming planets, a connection should exist between the orbital radii of exoplanets and disk cavity radii according to planet-disk interaction models (Pinilla et al. 2012) unless planets migrate from their birth location. Planets are expected to clear cavities up to twice their orbital radius. We investigate this relation in Fig. 11. Only cavity radii $>2 \mathrm{AU}$ are considered. Both samples are heavily biased, in particular, there is a lack of planets on orbits larger than $5 \mathrm{AU}$ 

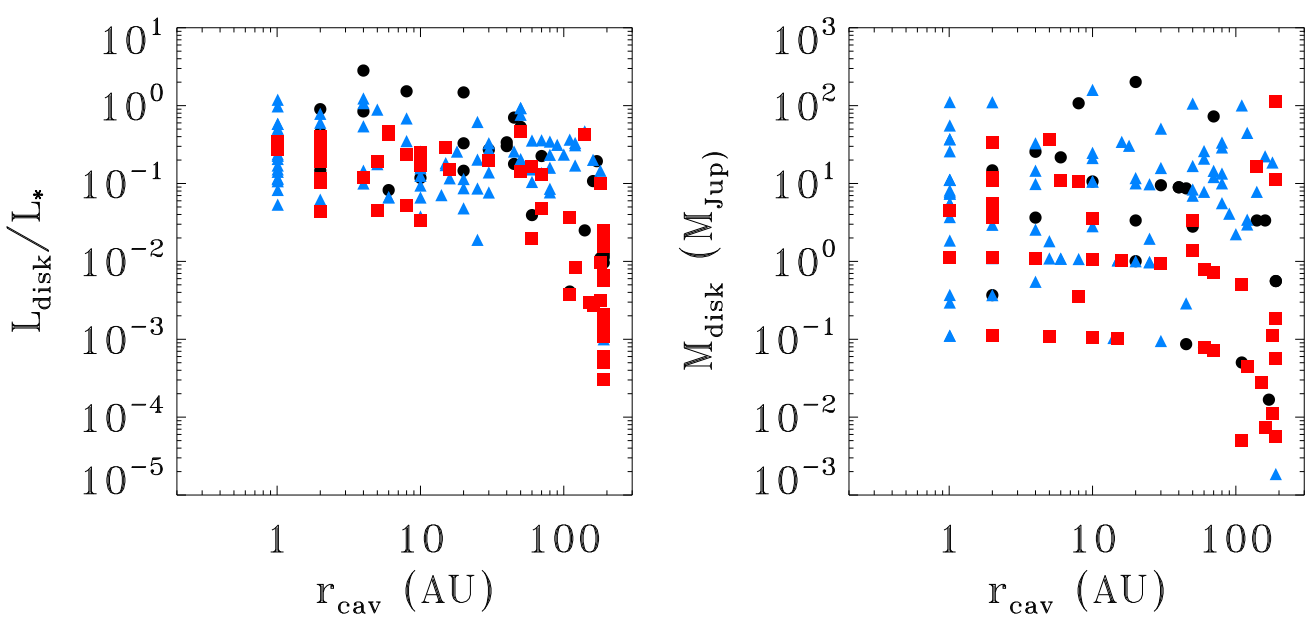

Fig. 10. Relation between cavity radius and $L_{\text {disk }} / L_{*}$ and disk mass. Blue triangles indicate accretors, red squares indicate nonaccretors, black circles denote unknown.

(radial velocity limit), whereas disk cavity radii cannot be detected below 2 AU. The exoplanet data is only fairly complete for $r<1 \mathrm{AU}$ (from Kepler), whereas there is no information about transition disk cavity radii at this limit. Therefore, we cannot test any connection with the current data, but as both disk and exoplanet will start to fill the critical 2-10 AU range in the coming years it will be important to search for such a relation. A trend is seen between cavity radius and stellar mass (blue and red points in Fig. 11) as seen in previous works (Kim et al. 2009; Merín et al. 2010).

\subsection{Transition disks within the full YSO sample}

A total number of 133 transition disk candidates (ML, MS, LL or LS category) is confirmed through the SED modeling, and 108 of these are from one of the main Spitzer catalogs (c2d, GB or Taurus) of YSOs in nearby star-forming regions. These three catalogs add up to 3331 YSOs, out of which 1387 are classified as disks (Class II objects). This means that approximately $8 \%$ of the disks in star-forming regions are expected to be transition disks, considering only the targets from the Spitzer catalogs. Assuming a Class II lifetime of 3 Myr (Dunham et al. 2015; Ribas et al. 2015), this implies a transition disk lifetime of $0.24 \mathrm{Myr}$, assuming that disk evolution is continuous over the timespan of the Class II lifetime. More specifically, assuming that transition disks evolve by dissipating mass, the first stage of massive disks (MS+ML, 45 targets) is about $90 \mathrm{kyr}$, followed by a longer period of low-mass disks (LS+LL, 63 targets) of $150 \mathrm{kyr}$. Although it is tempting to conclude that disk dissipation is rapid (Williams \& Cieza 2011; Owen 2016), it should be noted that about half of the low-mass disks in the sample are still accreting (see Fig. 4) and disk mass alone cannot be used as a robust measurement of transition disk evolution.

\section{Summary}

The main results of this work are summarized as follows.

1. A large sample of $\sim 200$ transition disk candidates is presented and analyzed. Candidates are primarily selected from the Spitzer catalogs using the color criteria from Merín et al. (2010) with some additional targets from the literature.

2. The Spitzer data of the targets have been complemented with new submillimeter fluxes, Herschel-PACS archival data, and

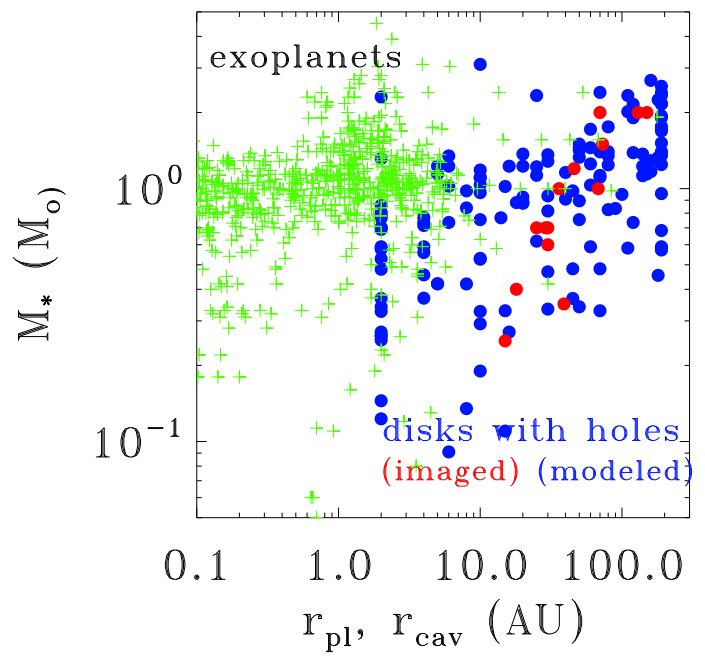

Fig. 11. Comparison of exoplanet orbital radii with transition disk cavity radii. Only cavity radii $>2 \mathrm{AU}$ are considered. Exoplanet orbital radii are indicated as green crosses, disk cavities with blue circles for SED modeling of this study and red circles for millimeter imaging.

optical/near-infrared spectroscopy for spectral typing and accretion properties.

3. All targets are analyzed using RADMC-3D modeling with a limited number of parameters: disk mass, inner disk mass, scale height and flaring, and disk cavity radius, where the latter is the main parameter of interest.

4. The derived cavity sizes are consistent with imaging/modeling results in the literature where available.

5. Using the derived properties, the disks are categorized in disks without holes, large/small holes and massive/low-mass disks, and very low scale height disks. The latter are likely debris disks. A large fraction ( $23 \%)$ of the targets falls in the category of disks with large holes, including several previously imaged disks.

6. Based on this classification, 133 targets $(72 \%$ of the sample) are disks with cavities, including about 70 new transition disk candidates that had not been identified before.

7. The color criteria are evaluated and compared for the targets in the Cieza studies (Cieza et al. 2010, 2012b; Romero et al. 2012). In general, our color criteria are a proper tool to select transition disk candidates. 
8. The cavity radius increases with stellar mass.

9. The sample list provides a large number of transition disk candidates that are suitable for follow-up observations with ALMA.

Acknowledgements. The authors would like to thank C. Manara for useful discussions and M. McClure for providing the IRS spectrum on DoAr28. N.M. is supported by the Netherlands Research School for Astronomy (NOVA). Astrochemistry in Leiden is supported by the Netherlands Research School for Astronomy (NOVA), by a Royal Netherlands Academy of Arts and Sciences (KNAW) professor prize, and by the European Union A-ERC grant 291141 CHEMPLAN This publication makes use of data products from the Wide-field Infrared Survey Explorer, which is a joint project of the University of California, Los Angeles, and the Jet Propulsion Laboratory/California Institute of Technology, funded by the National Aeronautics and Space Administration.

\section{References}

Alcala, J. M., Krautter, J., Schmitt, J. H. M. M., et al. 1995, A\&AS, 114, 109 Alcalá, J. M., Spezzi, L., Chapman, N., et al. 2008, ApJ, 676, 427 Alcalá, J. M., Natta, A., Manara, C. F., et al. 2014, A\&A, 561, A2 Alexander, R. D., Clarke, C. J., \& Pringle, J. E. 2006, MNRAS, 369, 216 Andrews, S. M., \& Williams, J. P. 2005, ApJ, 631, 1134 Andrews, S. M., \& Williams, J. P. 2007a, ApJ, 671, 1800 Andrews, S. M., \& Williams, J. P. 2007b, ApJ, 659, 705

Andrews, S. M., Wilner, D. J., Hughes, A. M., Qi, C., \& Dullemond, C. P. 2009, ApJ, 700, 1502

Andrews, S. M., Wilner, D. J., Hughes, A. M., Qi, C., \& Dullemond, C. P. 2010 ApJ, 723, 1241

Andrews, S. M., Wilner, D. J., Espaillat, C., et al. 2011, ApJ, 732, 42

Andrews, S. M., Wilner, D. J., Hughes, A. M., et al. 2012, ApJ, 744, 162

Andrews, S. M., Rosenfeld, K. A., Kraus, A. L., \& Wilner, D. J. 2013, ApJ, 771 , 129

Artymowicz, P., \& Lubow, S. H. 1994, ApJ, 421, 651

Artymowicz, P., \& Lubow, S. H. 1996, ApJ, 467, L77

Baraffe, I., Chabrier, G., Allard, F., \& Hauschildt, P. H. 1998, A\&A, 337, 403

Biller, B., Allers, K., Liu, M., Close, L. M., \& Dupuy, T. 2011, ApJ, 730, 39

Biller, B., Lacour, S., Juhász, A., et al. 2012, ApJ, 753, L38

Birnstiel, T., Dullemond, C. P., \& Brauer, F. 2010, A\&A, 513, A79

Bouwman, J., Lawson, W. A., Juhász, A., et al. 2010, ApJ, 723, L243

Broekhoven-Fiene, H., Matthews, B. C., Harvey, P. M., et al. 2014, ApJ, 786, 37

Brown, J. M., Blake, G. A., Dullemond, C. P., et al. 2007, ApJ, 664, L107

Brown, J. M., Blake, G. A., Qi, C., Dullemond, C. P., \& Wilner, D. J. 2008, ApJ, 675, L109

Brown, J. M., Blake, G. A., Qi, C., et al. 2009, ApJ, 704, 496

Brown, J. M., Herczeg, G. J., Pontoppidan, K. M., \& van Dishoeck, E. F. 2012, ApJ, 744, 116

Bruderer, S., van der Marel, N., van Dishoeck, E. F., \& van Kempen, T. A. 2014, A\&A, 562, A26

Bustamante, I., Merín, B., Ribas, Á., et al. 2015, A\&A, 578, A23

Calvet, N., D’Alessio, P., Hartmann, L., et al. 2002, ApJ, 568, 1008

Calvet, N., Muzerolle, J., Briceño, C., et al. 2004, AJ, 128, 1294

Canovas, H., Schreiber, M. R., Cáceres, C., et al. 2015, ApJ, 805, 21

Carpenter, J. M., Bouwman, J., Silverstone, M. D., et al. 2008, ApJS, 179, 423

Casassus, S., van der Plas, G., M, S. P., et al. 2013, Nature, 493, 191

Castelli, F., \& Kurucz, R. L. 2004, Proc. IAU Symp., 210, A20

Chiang, E. I., \& Goldreich, P. 1997, ApJ, 490, 368

Cieza, L., Padgett, D. L., Stapelfeldt, K. R., et al. 2007, ApJ, 667, 308

Cieza, L. A., Swift, J. J., Mathews, G. S., \& Williams, J. P. 2008, ApJ, 686, L115

Cieza, L. A., Schreiber, M. R., Romero, G. A., et al. 2010, ApJ, 712, 925

Cieza, L. A., Mathews, G. S., Williams, J. P., et al. 2012a, ApJ, 752, 75

Cieza, L. A., Schreiber, M. R., Romero, G. A., et al. 2012b, ApJ, 750, 157

Cody, A. M., Stauffer, J., Baglin, A., et al. 2014, AJ, 147, 82

Comerón, F. 2008, in Handbook of Star Forming Regions, Vol. II, ed B. Reipurth, 295

Comerón, F. 2012, A\&A, 537, A97

Comerón, F., Reipurth, B., Henry, A., \& Fernández, M. 2004, A\&A, 417, 583

Comerón, F., Spezzi, L., \& López Martí, B. 2009, A\&A, 500, 1045

Cortes, S. R., Meyer, M. R., Carpenter, J. M., et al. 2009, ApJ, 697, 1305

Currie, T., \& Kenyon, S. J. 2009, AJ, 138, 703

Currie, T., \& Sicilia-Aguilar, A. 2011, ApJ, 732, 24

Cutri, R. M., Skrutskie, M. F., van Dyk, S., et al. 2003, VizieR Online Data Catalog: II/246

D’Alessio, P., Hartmann, L., Calvet, N., et al. 2005, ApJ, 621, 461

Dullemond, C. P., \& Dominik, C. 2004, A\&A, 417, 159
Dullemond, C. P., \& Dominik, C. 2005, A\&A, 434, 971

Dullemond, C. P., Dominik, C., \& Natta, A. 2001, ApJ, 560, 957

Dunham, M. M., Allen, L. E., Evans, II, N. J., et al. 2015, ApJS, 220, 11

Dzib, S., Loinard, L., Mioduszewski, A. J., et al. 2010, ApJ, 718, 610

Dzib, S., Loinard, L., Rodríguez, L. F., Mioduszewski, A. J., \& Torres, R. M. 2011, ApJ, 733, 71

Enoch, M. L., Young, K. E., Glenn, J., et al. 2006, ApJ, 638, 293

Erickson, K. L., Wilking, B. A., Meyer, M. R., et al. 2015, AJ, 149, 103

Espaillat, C., Calvet, N., D'Alessio, P., et al. 2007, ApJ, 670, L135

Espaillat, C., Furlan, E., D'Alessio, P., et al. 2011, ApJ, 728, 49

Espaillat, C., Muzerolle, J., Najita, J., et al. 2014, Protostars and Planets VI, 497

Evans, II, N. J., Dunham, M. M., Jørgensen, J. K., et al. 2009, ApJS, 181, 321

Flaherty, K. M., \& Muzerolle, J. 2010, ApJ, 719, 1733

Fukagawa, M., Tsukagoshi, T., Momose, M., et al. 2013, PASJ, 65, L14

Furlan, E., Hartmann, L., Calvet, N., et al. 2006, ApJS, 165, 568

Furlan, E., Luhman, K. L., Espaillat, C., et al. 2011, ApJS, 195, 3

Garcia Lopez, R., Natta, A., Testi, L., \& Habart, E. 2006, A\&A, 459, 837

Ghez, A. M., McCarthy, D. W., Patience, J. L., \& Beck, T. L. 1997, ApJ, 481, 378

Gräfe, C., \& Wolf, S. 2013, A\&A, 552, A88

Guenther, E. W., Esposito, M., Mundt, R., et al. 2007, A\&A, 467, 1147

Hamuy, M., Walker, A. R., Suntzeff, N. B., et al. 1992, PASP, 104, 533

Hardy, A., Caceres, C., Schreiber, M. R., et al. 2015, A\&A, 583, A66

Harvey, P., Merín, B., Huard, T. L., et al. 2007, ApJ, 663, 1149

Harvey, P. M., Huard, T. L., Jørgensen, J. K., et al. 2008, ApJ, 680, 495

Hatchell, J., Terebey, S., Huard, T., et al. 2012, ApJ, 754, 104

Henning, T., Pfau, W., Zinnecker, H., \& Prusti, T. 1993, A\&A, 276, 129

Herczeg, G. J., \& Hillenbrand, L. A. 2014, ApJ, 786, 97

Hernández, J., Hartmann, L., Megeath, T., et al. 2007, ApJ, 662, 1067

Hernández, J., Morales-Calderon, M., Calvet, N., et al. 2010, ApJ, 722, 1226

Holland, W. S., Bintley, D., Chapin, E. L., et al. 2013, MNRAS, 430, 2513

Huélamo, N., Lacour, S., Tuthill, P., et al. 2011, A\&A, 528, L7

Huélamo, N., de Gregorio-Monsalvo, I., Macias, E., et al. 2015, A\&A, 575, L5

Hughes, A. M., Wilner, D. J., Qi, C., \& Hogerheijde, M. R. 2008, ApJ, 678, 1119

Hughes, A. M., Andrews, S. M., Espaillat, C., et al. 2009, ApJ, 698, 131

Hughes, A. M., Andrews, S. M., Wilner, D. J., et al. 2010, AJ, 140, 887

Indebetouw, R., Mathis, J. S., Babler, B. L., et al. 2005, ApJ, 619, 931

Ireland, M. J., \& Kraus, A. L. 2008, ApJ, 678, L59

Isella, A., Carpenter, J. M., \& Sargent, A. I. 2009, ApJ, 701, 260

Isella, A., Carpenter, J. M., \& Sargent, A. I. 2010a, ApJ, 714, 1746

Isella, A., Natta, A., Wilner, D., Carpenter, J. M., \& Testi, L. 2010b, ApJ, 725, 1735

Jensen, E. L. N., Mathieu, R. D., \& Fuller, G. A. 1996, ApJ, 458, 312

Jørgensen, J. K., Harvey, P. M., Evans, II, N. J., et al. 2006, ApJ, 645, 1246

Kenyon, S. J., \& Hartmann, L. 1995, ApJS, 101, 117

Kenyon, S. J., Gómez, M., \& Whitney, B. A. 2008, in Handbook of Star Forming Regions, Vol. I, ed. B. Reipurth, 405

Kim, K. H., Watson, D. M., Manoj, P., et al. 2009, ApJ, 700, 1017

Kirk, J. M., Ward-Thompson, D., Di Francesco, J., et al. 2009, ApJS, 185, 198

Kohler, R., \& Leinert, C. 1998, A\&A, 331, 977

Kovács, A. 2008, in SPIE Conf. Ser., 7020, 1

Kraus, A. L., \& Hillenbrand, L. A. 2009, ApJ, 703, 1511

Kraus, A. L., \& Ireland, M. J. 2012, ApJ, 745, 5

Kraus, A. L., Ireland, M. J., Martinache, F., \& Lloyd, J. P. 2008, ApJ, 679, 762

Lafrenière, D., Jayawardhana, R., Brandeker, A., Ahmic, M., \& van Kerkwijk, M. H. 2008, ApJ, 683, 844

Lawson, W. A., Lyo, A.-R., \& Muzerolle, J. 2004, MNRAS, 351, L39

Lebouteiller, V., Barry, D. J., Spoon, H. W. W., et al. 2011, ApJS, 196, 8

Leinert, C., Henry, T., Glindemann, A., \& McCarthy, Jr., D. W. 1997, A\&A, 325, 159

Lin, D. N. C., \& Papaloizou, J. 1979, MNRAS, 188, 191

Loinard, L., Torres, R. M., Mioduszewski, A. J., \& Rodríguez, L. F. 2008, ApJ, 675, L29

Lommen, D., Wright, C. M., Maddison, S. T., et al. 2007, A\&A, 462, 211

Lommen, D. J. P., van Dishoeck, E. F., Wright, C. M., et al. 2010, A\&A, 515, A77

Luhman, K. L. 2004, ApJ, 602, 816

Luhman, K. L. 2007, ApJS, 173, 104

Luhman, K. L., \& Muench, A. A. 2008, ApJ, 684, 654

Luhman, K. L., Allen, L. E., Allen, P. R., et al. 2008, ApJ, 675, 1375

Luhman, K. L., Allen, P. R., Espaillat, C., Hartmann, L., \& Calvet, N. 2010, ApJS, 186, 111

Mamajek, E. E., Lawson, W. A., \& Feigelson, E. D. 1999, ApJ, 516, L77

Manara, C. F., Testi, L., Natta, A., et al. 2014, A\&A, 568, A18

Manara, C. F., Fedele, D., Herczeg, G. J., \& Teixeira, P. S. 2016, A\&A, 585, A136

Mathews, G. S., Williams, J. P., \& Ménard, F. 2012, ApJ, 753, 59

McClure, M. K., Furlan, E., Manoj, P., et al. 2010, ApJS, 188, 75 
Megeath, S. T., Hartmann, L., Luhman, K. L., \& Fazio, G. G. 2005, ApJ, 634, L113

Megeath, S. T., Gutermuth, R., Muzerolle, J., et al. 2012, AJ, 144, 192

Mendigutía, I., Mora, A., Montesinos, B., et al. 2012, A\&A, 543, A59

Merín, B., Jørgensen, J., Spezzi, L., et al. 2008, ApJS, 177, 551

Merín, B., Brown, J. M., Oliveira, I., et al. 2010, ApJ, 718, 1200

Monnier, J. D., Tannirkulam, A., Tuthill, P. G., et al. 2008, ApJ, 681, L97

Mooley, K., Hillenbrand, L., Rebull, L., Padgett, D., \& Knapp, G. 2013, ApJ, 771,110

Muzerolle, J., Allen, L. E., Megeath, S. T., Hernández, J., \& Gutermuth, R. A. 2010, ApJ, 708, 1107

Najita, J. R., Strom, S. E., \& Muzerolle, J. 2007, MNRAS, 378, 369

Najita, J. R., Carr, J. S., Strom, S. E., et al. 2010, ApJ, 712, 274

Natta, A., Testi, L., Muzerolle, J., et al. 2004, A\&A, 424, 603

Natta, A., Testi, L., \& Randich, S. 2006, A\&A, 452, 245

Nguyen, D. C., Brandeker, A., van Kerkwijk, M. H., \& Jayawardhana, R. 2012, ApJ, 745, 119

Nuernberger, D., Chini, R., \& Zinnecker, H. 1997, A\&A, 324, 1036

Ohashi, N. 2008, Ap\&SS, 313, 101

Oke, J. B. 1990, AJ, 99, 1621

Oliveira, I., Merín, B., Pontoppidan, K. M., et al. 2009, ApJ, 691, 672

Oliveira, I., Pontoppidan, K. M., Merín, B., et al. 2010, ApJ, 714, 778

Oliveira, I., Merín, B., Pontoppidan, K. M., \& van Dishoeck, E. F. 2013, ApJ, 762,128

Olofsson, J., Szűcs, L., Henning, T., et al. 2013, A\&A, 560, A100

Ortiz-León, G. N., Loinard, L., Mioduszewski, A. J., et al. 2015, ApJ, 805, 9

Owen, J. E. 2016, PASA, 33, e005

Owen, J. E., \& Clarke, C. J. 2012, MNRAS, 426, L96

Padgett, D. L., Cieza, L., Stapelfeldt, K. R., et al. 2006, ApJ, 645, 1283

Padgett, D. L., Rebull, L. M., Stapelfeldt, K. R., et al. 2008, ApJ, 672, 1013

Pérez, L. M., Isella, A., Carpenter, J. M., \& Chandler, C. J. 2014, ApJ, 783, L13

Perez, S., Casassus, S., Ménard, F., et al. 2015, ApJ, 798, 85

Peterson, D. E., Caratti o Garatti, A., Bourke, T. L., et al. 2011, ApJS, 194, 43

Pickles, A. J. 1998, PASP, 110, 863

Piétu, V., Guilloteau, S., \& Dutrey, A. 2005, A\&A, 443, 945

Piétu, V., Dutrey, A., Guilloteau, S., Chapillon, E., \& Pety, J. 2006, A\&A, 460, L43

Pilbratt, G. L., Riedinger, J. R., Passvogel, T., et al. 2010, A\&A, 518, L1

Pinilla, P., Benisty, M., \& Birnstiel, T. 2012, A\&A, 545, A81

Poglitsch, A., Waelkens, C., Geis, N., et al. 2010, A\&A, 518, L2

Pott, J.-U., Perrin, M. D., Furlan, E., et al. 2010, ApJ, 710, 265

Quanz, S. P. 2015, Ap\&SS, 357, 148

Quanz, S. P., Amara, A., Meyer, M. R., et al. 2013, ApJ, 766, L1

Ratzka, T., Köhler, R., \& Leinert, C. 2005, A\&A, 437, 611

Rebollido, I., Merín, B., Ribas, Á., et al. 2015, A\&A, 581, A30

Rebull, L. M., Padgett, D. L., McCabe, C.-E., et al. 2010, ApJS, 186, 259

Reggiani, M., Quanz, S. P., Meyer, M. R., et al. 2014, ApJ, 792, L23

Revéret, V., André, P., Le Pennec, J., et al. 2014, in SPIE Conf. Ser., 9153, 5

Ribas, Á., Merín, B., Bouy, H., et al. 2013, A\&A, 552, A115
Ribas, Á., Bouy, H., \& Merín, B. 2015, A\&A, 576, A52

Rigliaco, E., Pascucci, I., Duchene, G., et al. 2015, ApJ, 801, 31

Romero, G. A., Schreiber, M. R., Cieza, L. A., et al. 2012, ApJ, 749, 79

Rosenfeld, K. A., Andrews, S. M., Wilner, D. J., Kastner, J. H., \& McClure, M. K. 2013, ApJ, 775, 136

Rosotti, G. P., Ercolano, B., Owen, J. E., \& Armitage, P. J. 2013, MNRAS, 430, 1392

Sallum, S., Follette, K. B., Eisner, J. A., et al. 2015, Nature, 527, 342

Salyk, C., Herczeg, G. J., Brown, J. M., et al. 2013, ApJ, 769, 21

Schisano, E., Covino, E., Alcalá, J. M., et al. 2009, A\&A, 501, 1013

Schuller, F. 2012, in SPIE Conf. Ser., 8452, 1

Sicilia-Aguilar, A., Henning, T., Juhász, A., et al. 2008, ApJ, 687, 1145

Sicilia-Aguilar, A., Bouwman, J., Juhász, A., et al. 2009, ApJ, 701, 1188

Siess, L., Dufour, E., \& Forestini, M. 2000, A\&A, 358, 593

Silverstone, M. D., Meyer, M. R., Mamajek, E. E., et al. 2006, ApJ, 639, 1138

Siringo, G., Kreysa, E., Kovács, A., et al. 2009, A\&A, 497, 945

Siringo, G., Kreysa, E., De Breuck, C., et al. 2010, The Messenger, 139, 20

Spezzi, L., Alcalá, J. M., Covino, E., et al. 2008, ApJ, 680, 1295

Spezzi, L., Vernazza, P., Merín, B., et al. 2011, ApJ, 730, 65

Stauffer, J., Cody, A. M., Baglin, A., et al. 2014, AJ, 147, 83

Strom, K. M., Strom, S. E., Edwards, S., Cabrit, S., \& Skrutskie, M. F. 1989, AJ, 97, 1451

Tang, Y.-W., Guilloteau, S., Piétu, V., et al. 2012, A\&A, 547, A84

Torres, C. A. O., Quast, G. R., Melo, C. H. F., \& Sterzik, M. F. 2008, in Handbook of Star Forming Regions, Vol. II, , ed. B. Reipurth, 757

van der Marel, N., van Dishoeck, E. F., Bruderer, S., et al. 2013, Science, 340, 1199

van der Marel, N., van Dishoeck, E. F., Bruderer, S., Pérez, L., \& Isella, A. 2015, A\&A, 579, A106

van der Marel, N., van Dishoeck, E. F., Bruderer, S., et al. 2016, A\&A, 585, A58

van Kempen, T. A., van Dishoeck, E. F., Salter, D. M., et al. 2009, A\&A, 498, 167

Venuti, L., Bouvier, J., Flaccomio, E., et al. 2014, A\&A, 570, A82

Verhoeff, A. P., Min, M., Pantin, E., et al. 2011, A\&A, 528, A91

Vicente, S., Merín, B., Hartung, M., et al. 2011, A\&A, 533, A135

Wahhaj, Z., Cieza, L., Koerner, D. W., et al. 2010, ApJ, 724, 835

White, R. J., \& Basri, G. 2003, ApJ, 582, 1109

White, R. J., \& Ghez, A. M. 2001, ApJ, 556, 265

Wilking, B. A., Meyer, M. R., Robinson, J. G., \& Greene, T. P. 2005, AJ, 130, 1733

Williams, J. P., \& Cieza, L. A. 2011, ARA\&A, 49, 67

Winston, E., Megeath, S. T., Wolk, S. J., et al. 2009, AJ, 137, 4777

Wright, E. L., Eisenhardt, P. R. M., Mainzer, A. K., et al. 2010, AJ, 140, 1868

Young, K. E., Young, C. H., Lai, S.-P., Dunham, M. M., \& Evans, II, N. J. 2015, AJ, 150, 40

Zacharias, N., Monet, D. G., Levine, S. E., et al. 2005, VizieR Online Data Catalog: I/297

Zhang, K., Isella, A., Carpenter, J. M., \& Blake, G. A. 2014, ApJ, 791, 42 


\section{Appendix A: Additional tables}

Table A.1. Sample of transition disk candidates.

\begin{tabular}{llllllllll}
\hline \hline ID & Target & RA & Dec & Reg $^{a}$ & $\begin{array}{l}\mathrm{d}^{b} \\
(\mathrm{pc})\end{array}$ & Color $^{c}$ & $\begin{array}{l}\text { Origin } \\
\text { data }\end{array}$ & Prev. $^{d}$ & Ref \\
\hline 1 & TCha & 115713.53 & -792131.5 & $\epsilon$ Cha & 108 & {$[\mathrm{~L}]$} & Other & Imag. & 1,2 \\
2 & RECX11 & 084701.80 & -785935.2 & $\eta$ Cha & 97 & - & Other & TD & 3,4 \\
3 & RECX5 & 084227.09 & -785747.9 & $\eta$ Cha & 97 & {$[\mathrm{~A}]$} & Other & PF & 5,4 \\
4 & CHXR22E & 110713.30 & -774349.9 & ChaI & 160 & - & Other & TD & 6,7 \\
5 & ISO52 & 110442.58 & -774157.1 & ChaI & 160 & {$[\mathrm{~B}]$} & GB & V & 8 \\
$\ldots$ & & & & & & & & & \\
\hline
\end{tabular}

Notes. The full table is available at the CDS. ${ }^{(a)}$ Full names of the regions: Cha $=$ Chamaeleon, Lup $=$ Lupus, CrA $=$ Corona Australis, Oph $=$ Ophiuchus, UppS $=$ Upper Sco, Oph = Ophiuchus, Tau $=$ Taurus, Aur = Auriga, Per $=$ Perseus, $\mathrm{TWH}=\mathrm{TW}$ Hydrae, Isol. $=$ Isolated. ${ }^{(b)}$ Distances were adopted from the literature as follows: 120 pc for Oph (Loinard et al. 2008), 150 pc for Lup I, IV, V, and VI and 200 pc for LupIII (Comerón 2008; Comerón et al. 2009), 250 pc for Per (Jørgensen et al. 2006), 140 pc for Tau (Kenyon et al. 2008), 450 pc for Aur (Broekhoven-Fiene et al. 2014), 145 pc for Upp Sco (Carpenter et al. 2008), 150 pc for Corona Australis (Sicilia-Aguilar et al. 2008), 109 pc for $\epsilon$ Cha (Torres et al. 2008), $160 \mathrm{pc}$ for ChaI (Kim et al. 2009), 180 pc for ChaII (Alcalá et al. 2008), 97 pc for $\eta$ Cha (Mamajek et al. 1999), and 415 pc for Ser (Dzib et al. 2010). ${ }^{(c)}$ An asterix $(*)$ indicates this target was part of the M10 sample. ${ }^{(d)}$ The full explanation of previous classifications is as follows, according to their recording papers. 1) Cieza et al. (2010): "PF"=Planet-forming disk, "GG"=Grain-growth dominated disk, "PE"=Photoevaportive disk. 2) Merín et al. (2008), Harvey et al. (2007): "L"=low-infrared excess or anemic disk, "H"=high-infrared excess, "T"=T Tauri-like infrared excess. 3) Others: "PTD" = pretransitional disk, "V" = seesaw variability.

References. 1) Wahhaj et al. (2010), 2) Huélamo et al. (2015), 3) Megeath et al. (2005), 4) Sicilia-Aguilar et al. (2009), 5) Bouwman et al. (2010), 6) Kim et al. (2009), 7) Luhman et al. (2008), 8) Espaillat et al. (2011).

Table A.2. Stellar parameters.

\begin{tabular}{lllllllllll}
\hline \hline ID & Name & SpT & $\begin{array}{l}T_{\text {eff }} \\
(\mathrm{K})\end{array}$ & $\begin{array}{l}A_{V} \\
(\mathrm{mag})\end{array}$ & $\begin{array}{l}L_{*} \\
\left(L_{\odot}\right)\end{array}$ & $\begin{array}{l}M_{*} \\
\left(M_{\odot}\right)\end{array}$ & $\begin{array}{l}\mathrm{EW}[\mathrm{H} \alpha] \\
(\AA)\end{array}$ & $\begin{array}{l}\mathrm{FW} 10 \%[\mathrm{H} \alpha]^{a} \\
\left(\mathrm{~km} \mathrm{~s}^{-1}\right)\end{array}$ & $\begin{array}{l}\text { Accretion } \\
(\mathrm{Y} / \mathrm{N})\end{array}$ & Ref \\
\hline 1 & TCha & K0 & 5250 & 2 & 1.34 & 1.1 & - & 400 & $\mathrm{Y}$ & 1,2 \\
2 & RECX11 & K5 & 4350 & 0.4 & 0.73 & 1.2 & 4.4 & 330 & $\mathrm{Y}$ & 3 \\
3 & RECX5 & M4 & 3370 & 2.4 & 0.14 & 0.3 & 35 & 330 & $\mathrm{Y}$ & 3 \\
4 & CHXR22E & M3.5 & 3370 & 4.9 & 0.26 & 0.4 & - & - & $\mathrm{U}$ & 4 \\
5 & ISO52 & M4 & 3370 & 3 & 0.17 & 0.3 & $b$ & - & $\mathrm{N}$ & 5 \\
$\ldots$ & & & & & & & & & & \\
\hline
\end{tabular}

Notes. The full table is available at the CDS. ${ }^{(a)}$ We reversed the signs of the width of the H $\alpha$ line taken from Rebull et al. (2010) and Winston et al. (2009), as they list a negative value for emission and positive for absorption. ${ }^{(b)}$ The accretion properties were derived using a full X-shooter spectrum rather than only fitting the $\mathrm{H} \alpha$ line. ${ }^{(c)}$ The accretion properties were derived using other lines (e.g., Br $\gamma$ ). ${ }^{(d)}$ ' $\mathrm{Y}$ ' means accreting, 'N' means nonaccreting', and 'U' means unknown.

References. 1) Alcala et al. (1995), 2) Schisano et al. (2009), 3) Lawson et al. (2004), 4) Luhman (2007), 5) Manara et al. (2016).

Table A.3. Results of disk fitting procedure and classification.

\begin{tabular}{|c|c|c|c|c|c|c|c|c|c|c|}
\hline ID & Name & $\begin{array}{l}r_{\mathrm{cav}} \\
(\mathrm{AU})\end{array}$ & $\begin{array}{l}\Sigma_{c} \\
\left(\mathrm{~g} \mathrm{~cm}^{-2}\right)\end{array}$ & $\begin{array}{l}M_{\text {disk,fit }}{ }^{a} \\
\left(M_{\text {Jup }}\right)\end{array}$ & $\delta_{\text {dust }}$ & $h_{c}$ & $\psi$ & $\begin{array}{l}M_{\text {disk,mm }}{ }^{a} \\
\left(M_{\text {Jup }}\right)\end{array}$ & $L_{\text {disk }} / L_{*}$ & Classification $^{b}$ \\
\hline 1 & TCha & $140_{-10}^{+10}$ & $7 \mathrm{E}-02$ & 7.8 & $1 \mathrm{E}-02$ & 0.10 & $1 / 7$. & 3.7 & 0.467 & ML \\
\hline 2 & RECX11 & $6_{-4}^{+4}$ & $3 \mathrm{E}-03$ & 1.1 & $1 \mathrm{E}-02$ & 0.01 & $1 / 7$. & $<1.5$ & 0.067 & LS \\
\hline 3 & RECX5 & $10_{-2}^{+2}$ & $3 \mathrm{E}-04$ & 0.1 & $1 \mathrm{E}-04$ & 0.01 & $1 / 7$. & - & 0.038 & LL \\
\hline 4 & CHXR22E & $45_{-5}^{+15}$ & $3 \mathrm{E}-04$ & 0.1 & $1 \mathrm{E}-06$ & 0.01 & $2 / 7$ & - & 0.178 & LL \\
\hline 5 & ISO52 & $30_{-18}^{+50}$ & $3 \mathrm{E}-03$ & 0.9 & $1 \mathrm{E}-01$ & 0.05 & $2 / 7$ & - & 0.195 & LL \\
\hline
\end{tabular}

Notes. The full table is available at the CDS. ${ }^{(*)}$ Fit results are uncertain owing to unknown spectral type. ${ }^{(a)}$ Disk masses refer to the full disk mass that is computed assuming a gas-to-dust ratio of $100 .{ }^{(b)} \mathrm{NH}=$ disks without holes, ML = massive disks with large holes, MS = massive disks with small holes, $\mathrm{LL}=$ low-mass disks with large holes, $\mathrm{LS}=$ low-mass disks with small holes, and DD = low-mass disks with very low scale heights. See also definition in the text. 


\section{Appendix B: SEDs}
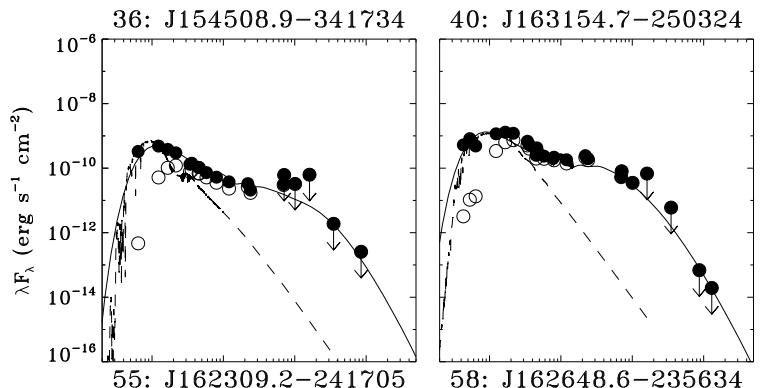

41: J163205.5-250236

53: J162435.2-242620
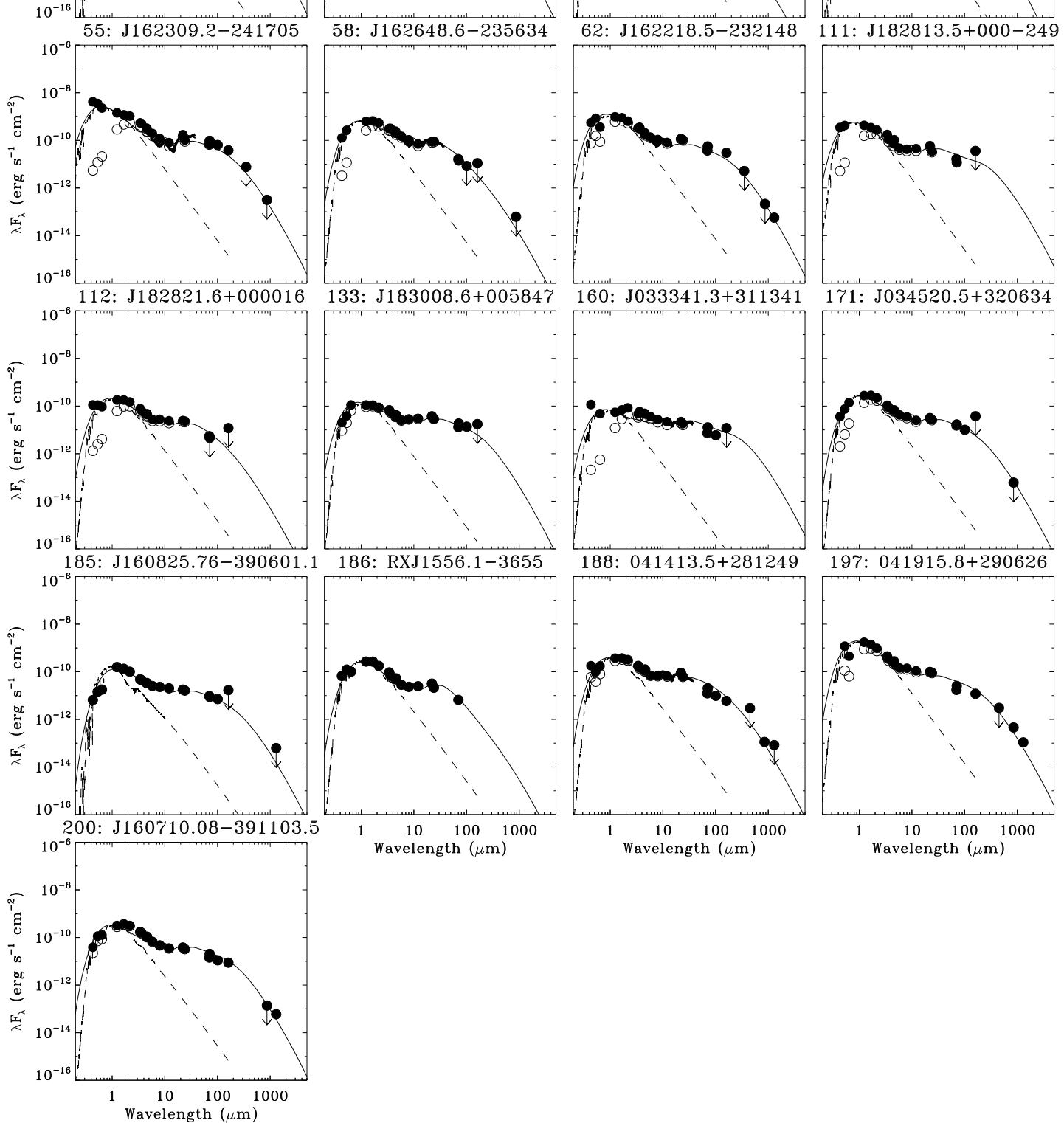

Fig. B.1. SEDs of disks without holes. In this and subsequent figures, the dashed line indicates the stellar spectrum, open circles denote observed fluxes before extinction correction, and filled circles indicate observed fluxes after extinction correction. 
A\&A 592, A126 (2016)
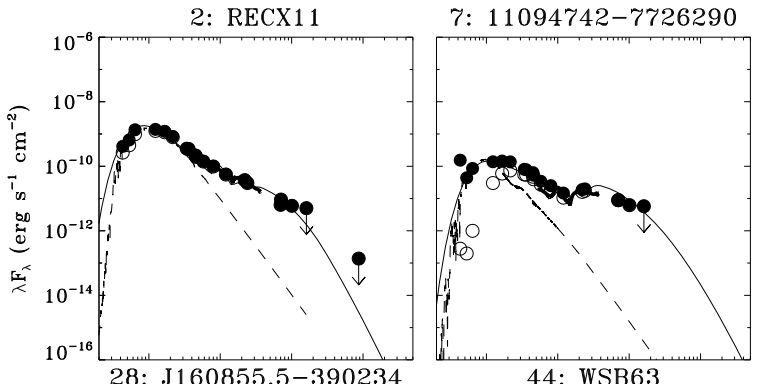

25: J160830.3-390611
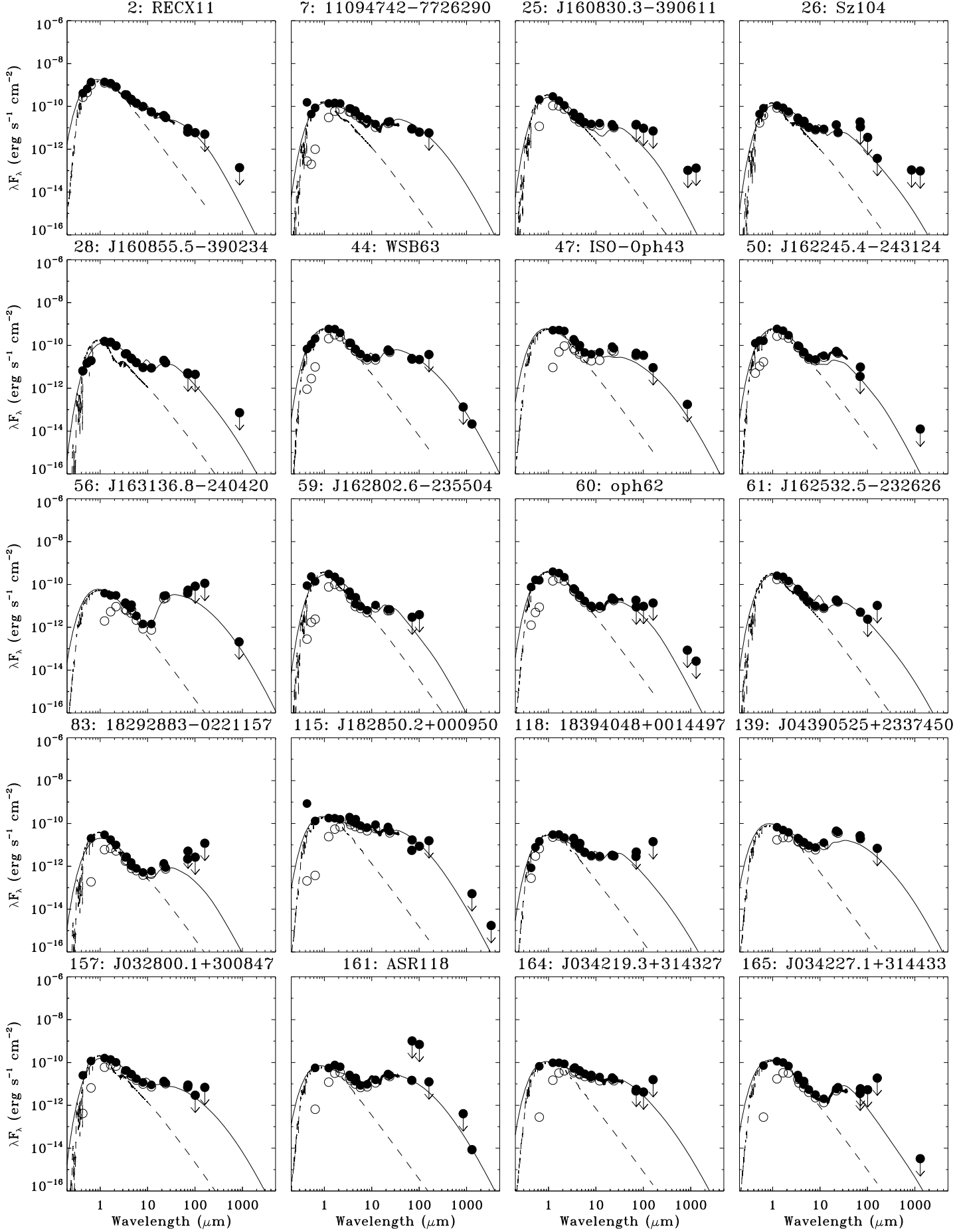

Fig. B.2. SEDs of low-mass disks with small holes. 
N. van der Marel et al.: The (w)hole survey

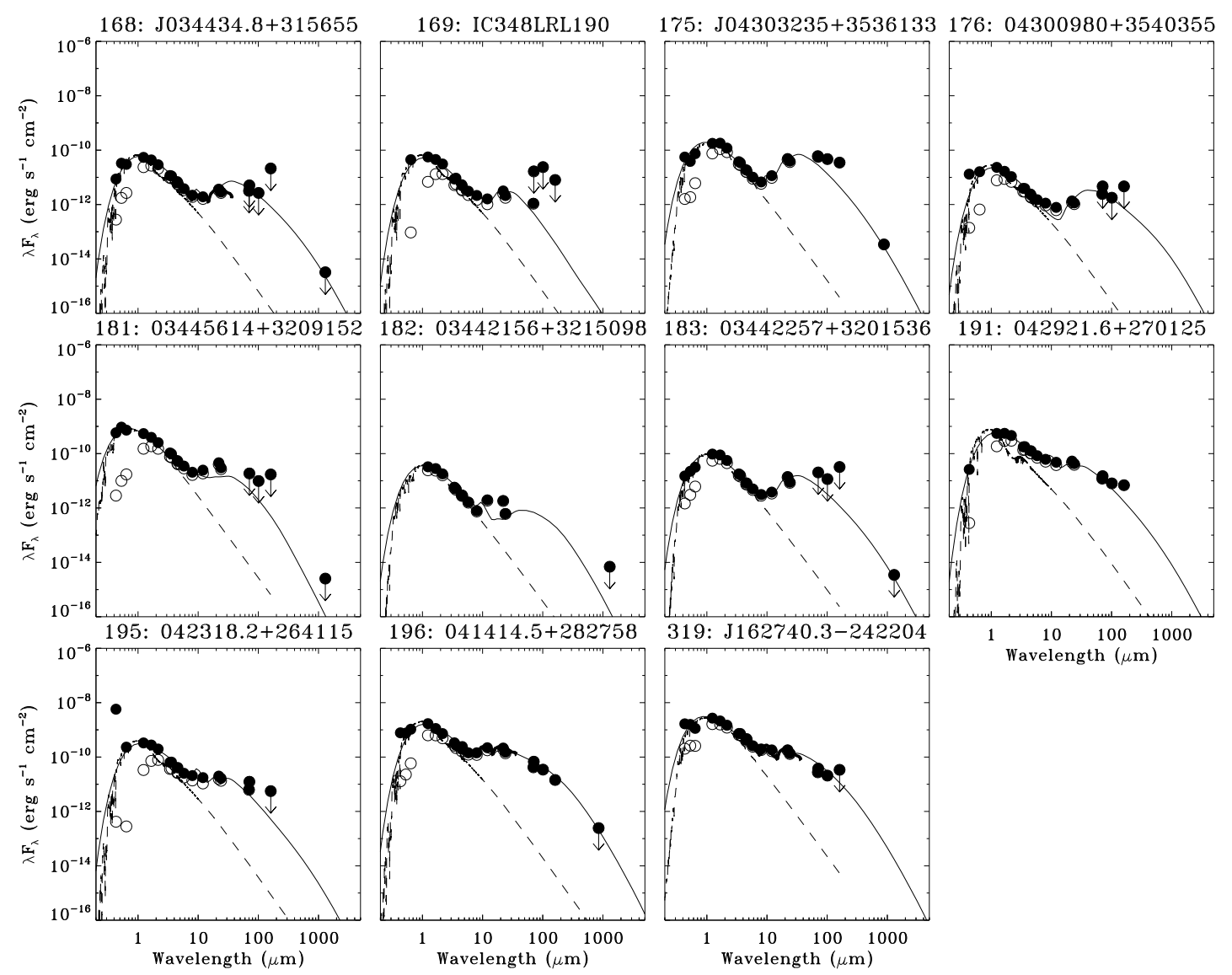

Fig. B.2. continued. 

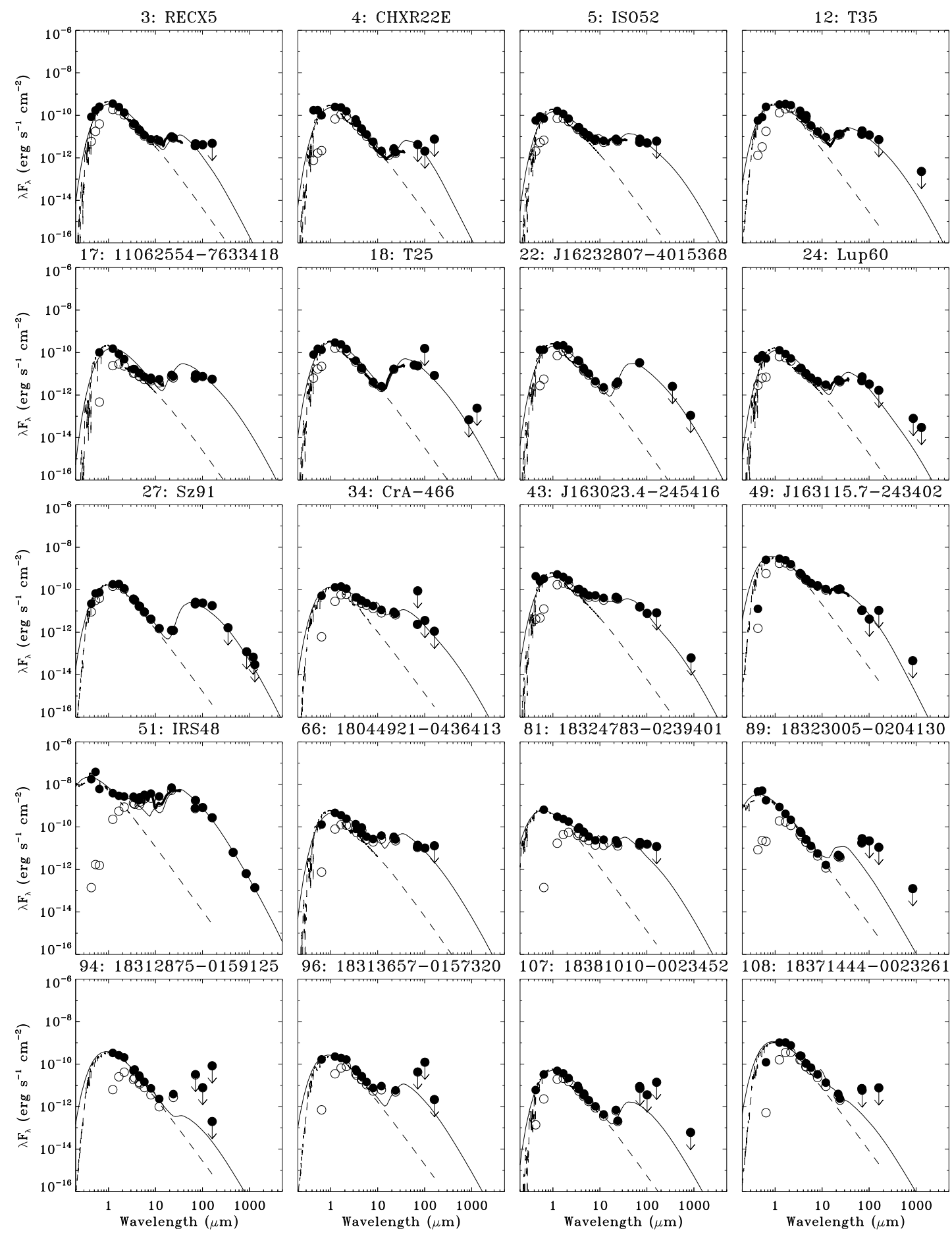

Fig. B.3. SEDs of low-mass disks with large holes. 

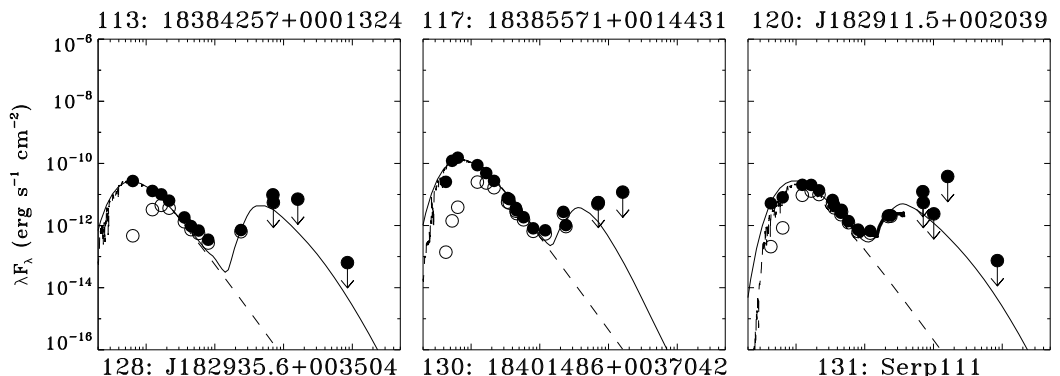

124: $\operatorname{serp} 22$
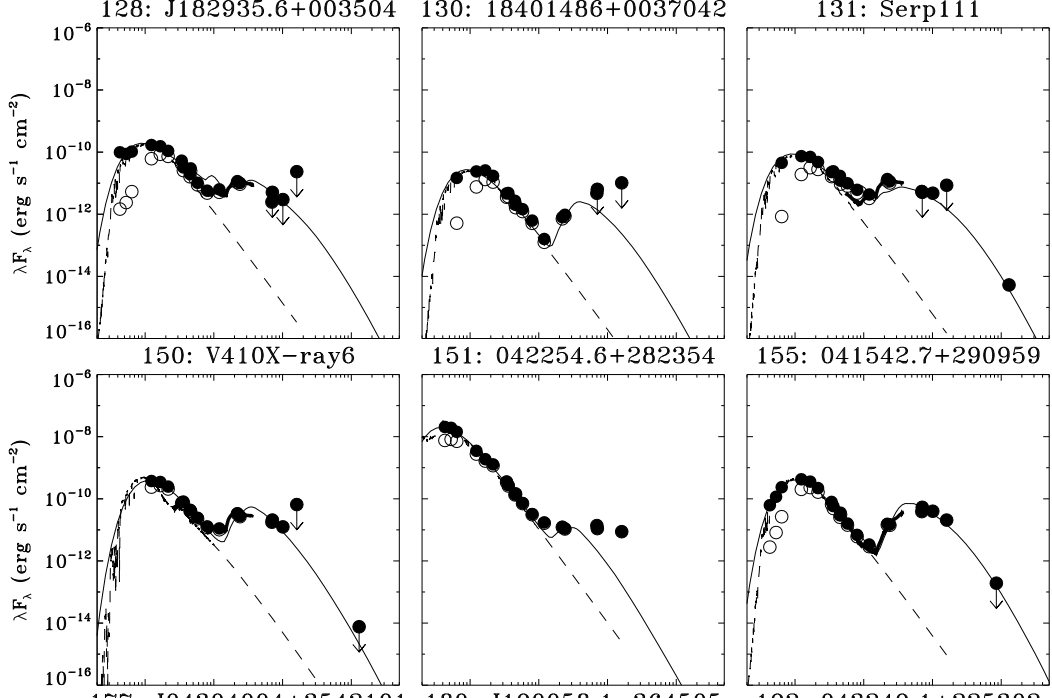

155: $041542.7+290959$

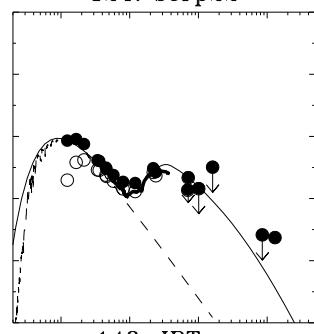

148: IPTau
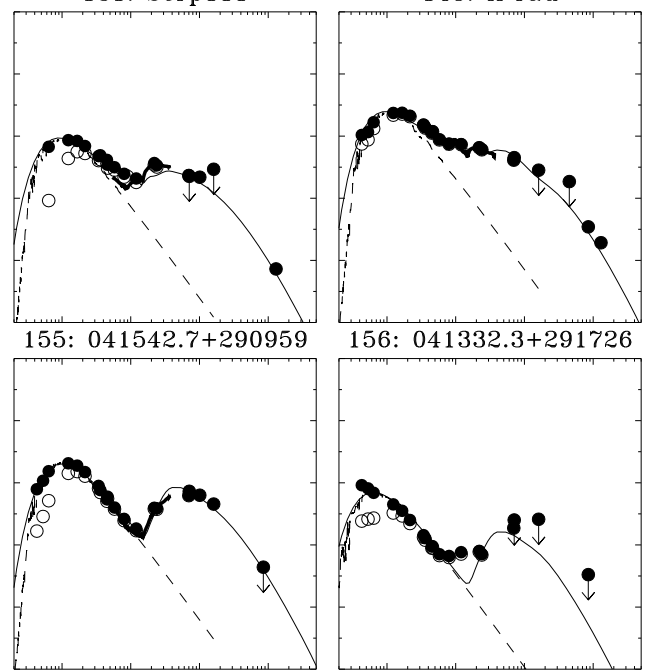

156: $041332.3+291726$
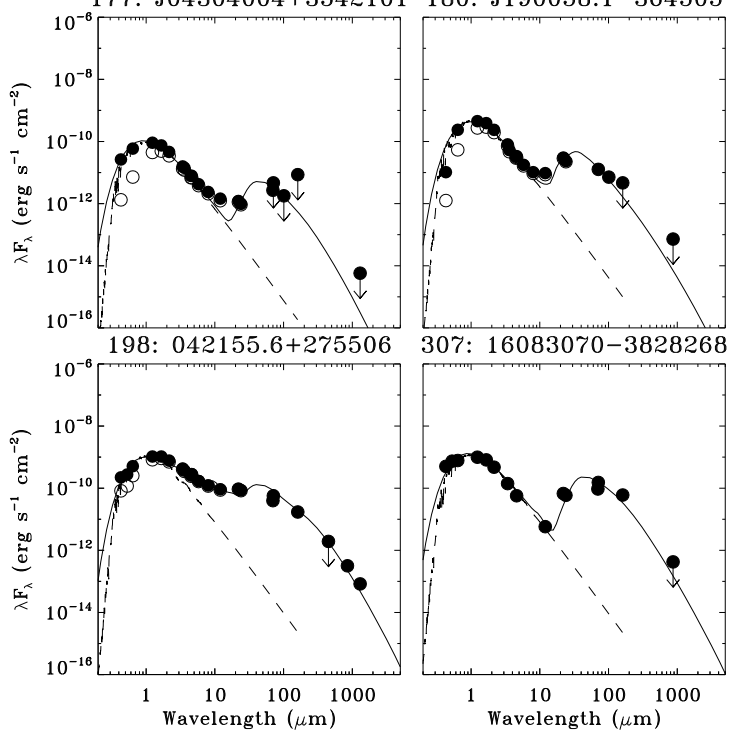

192: $043249.1+225302$
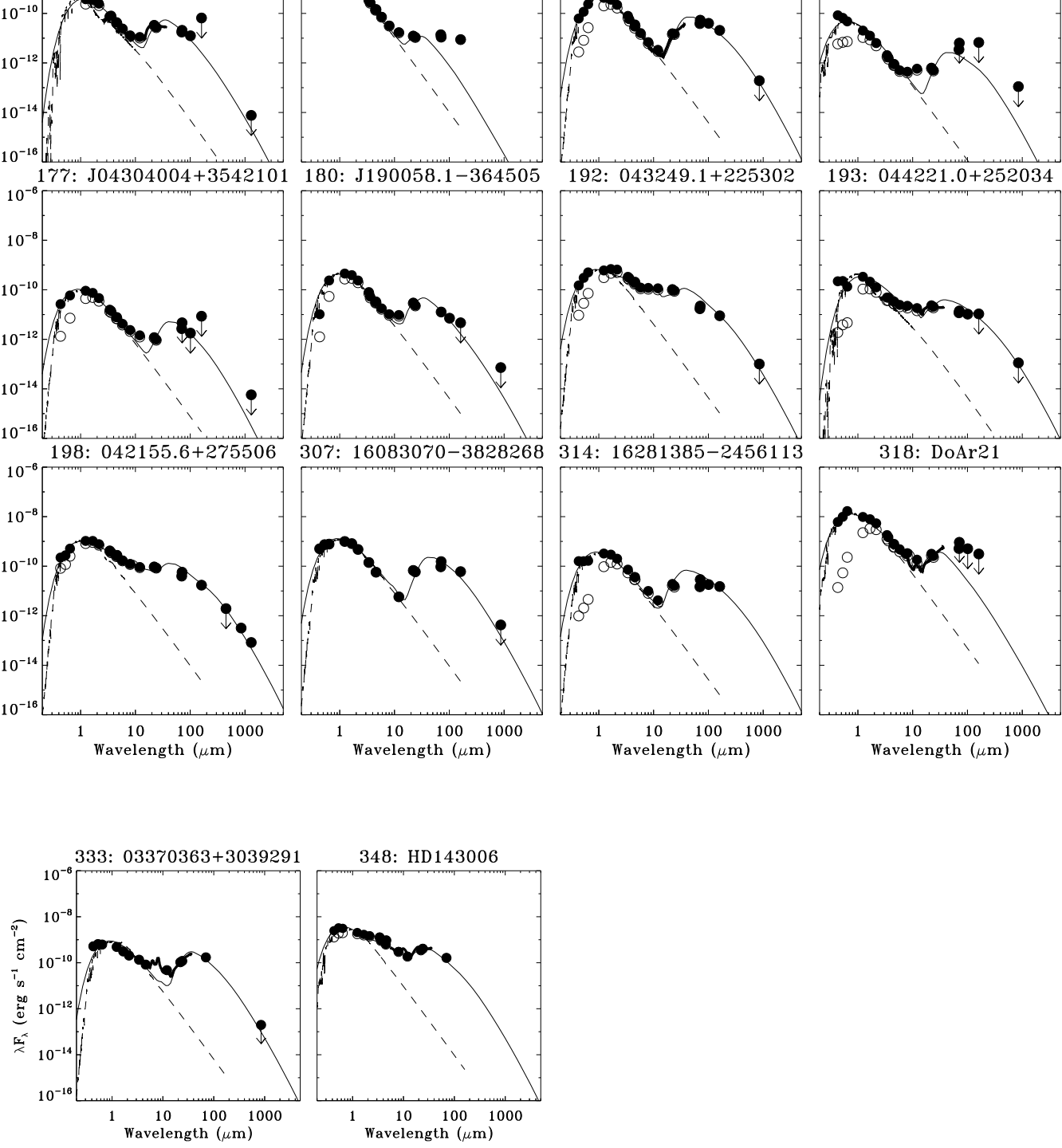

Fig. B.3. continued. 

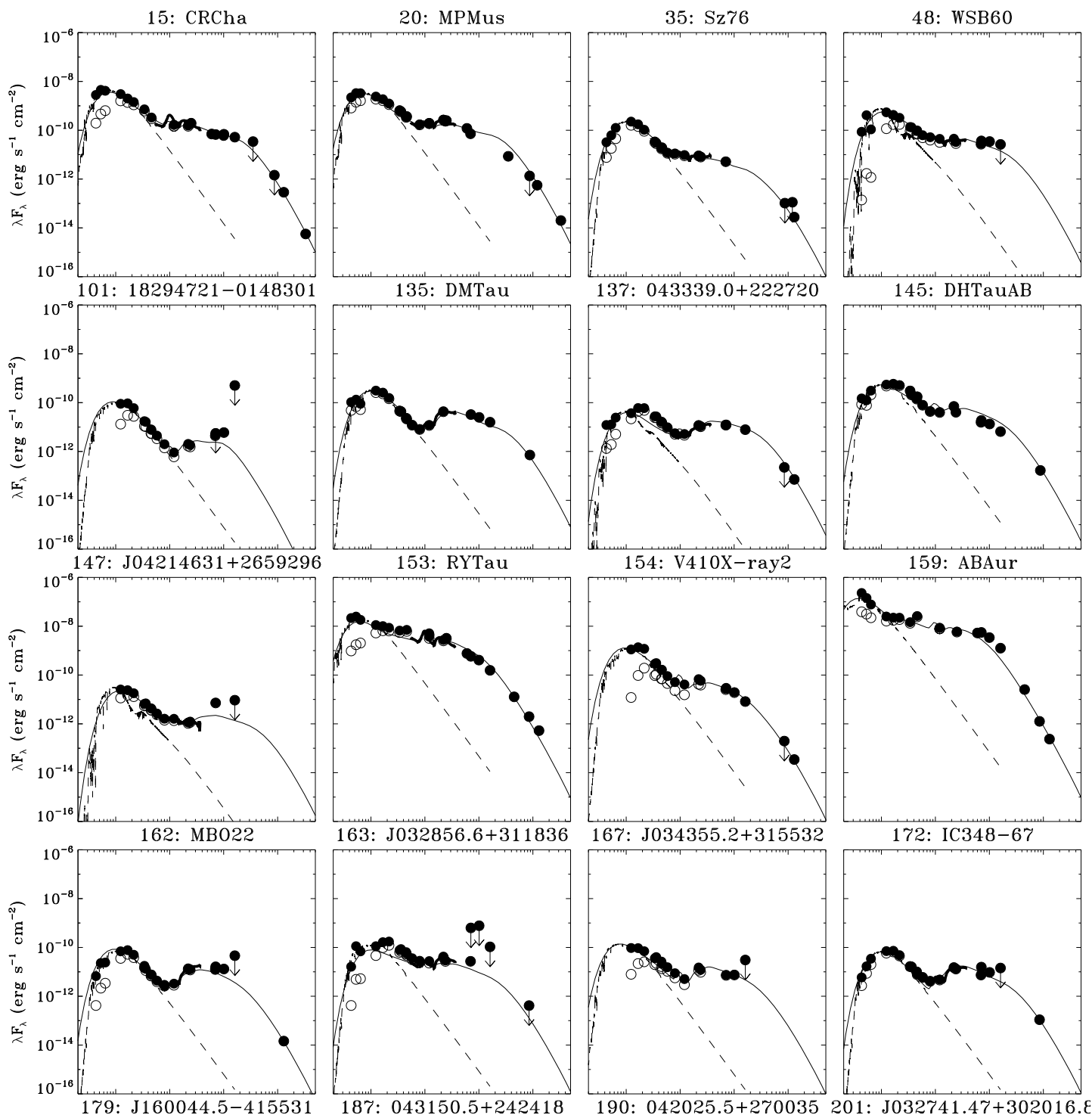

172: IC $348-67$
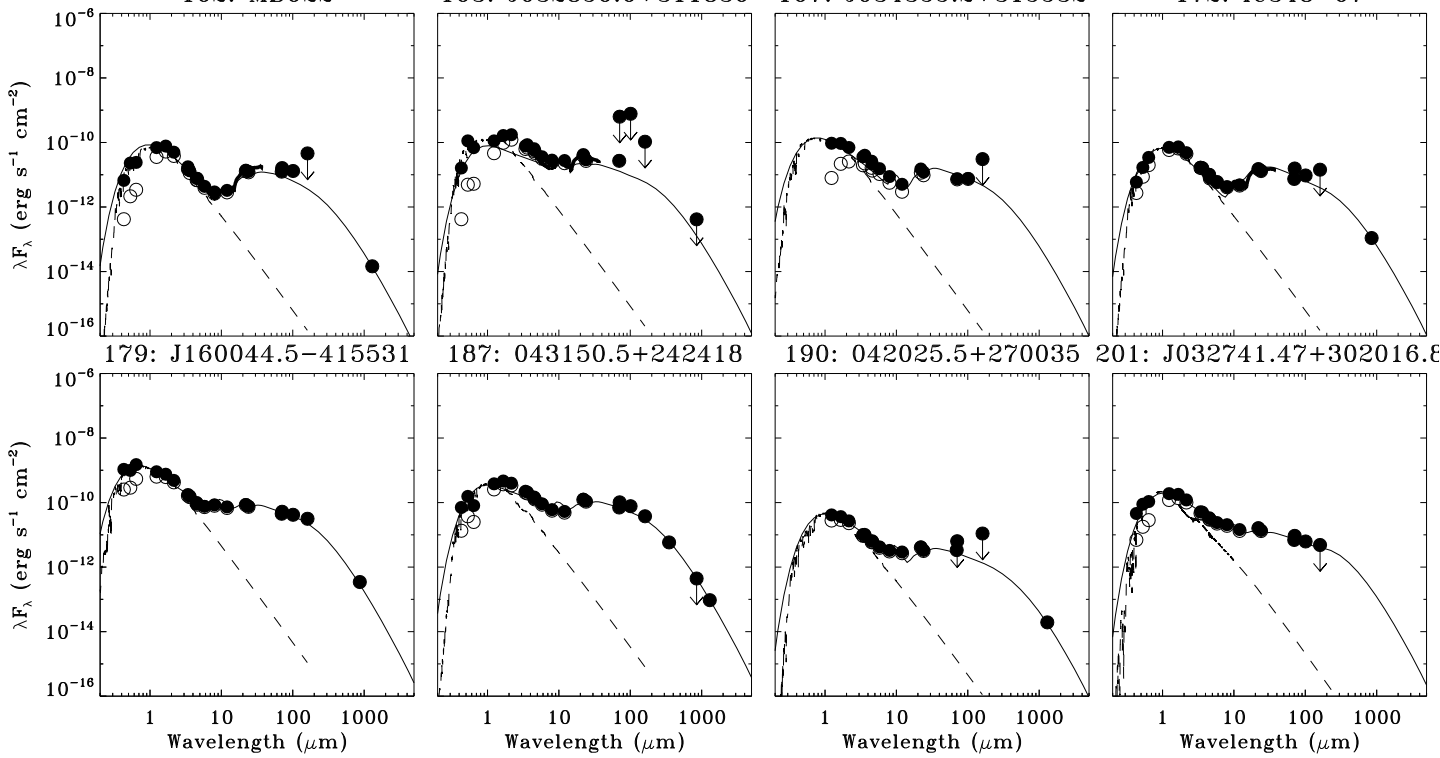

Fig. B.4. SEDs of massive disks with small holes. 
N. van der Marel et al.: The (w)hole survey

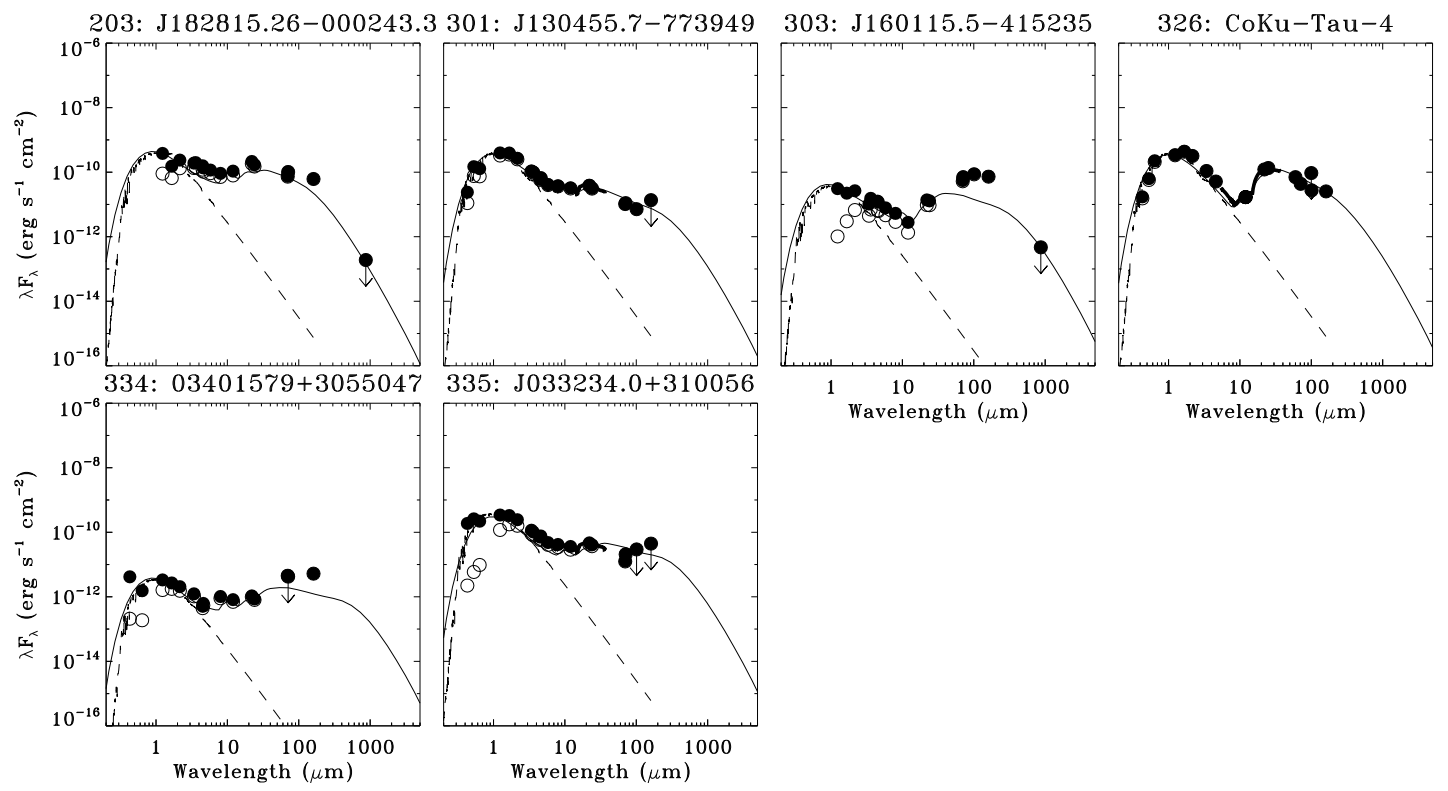

Fig. B.4. continued. 

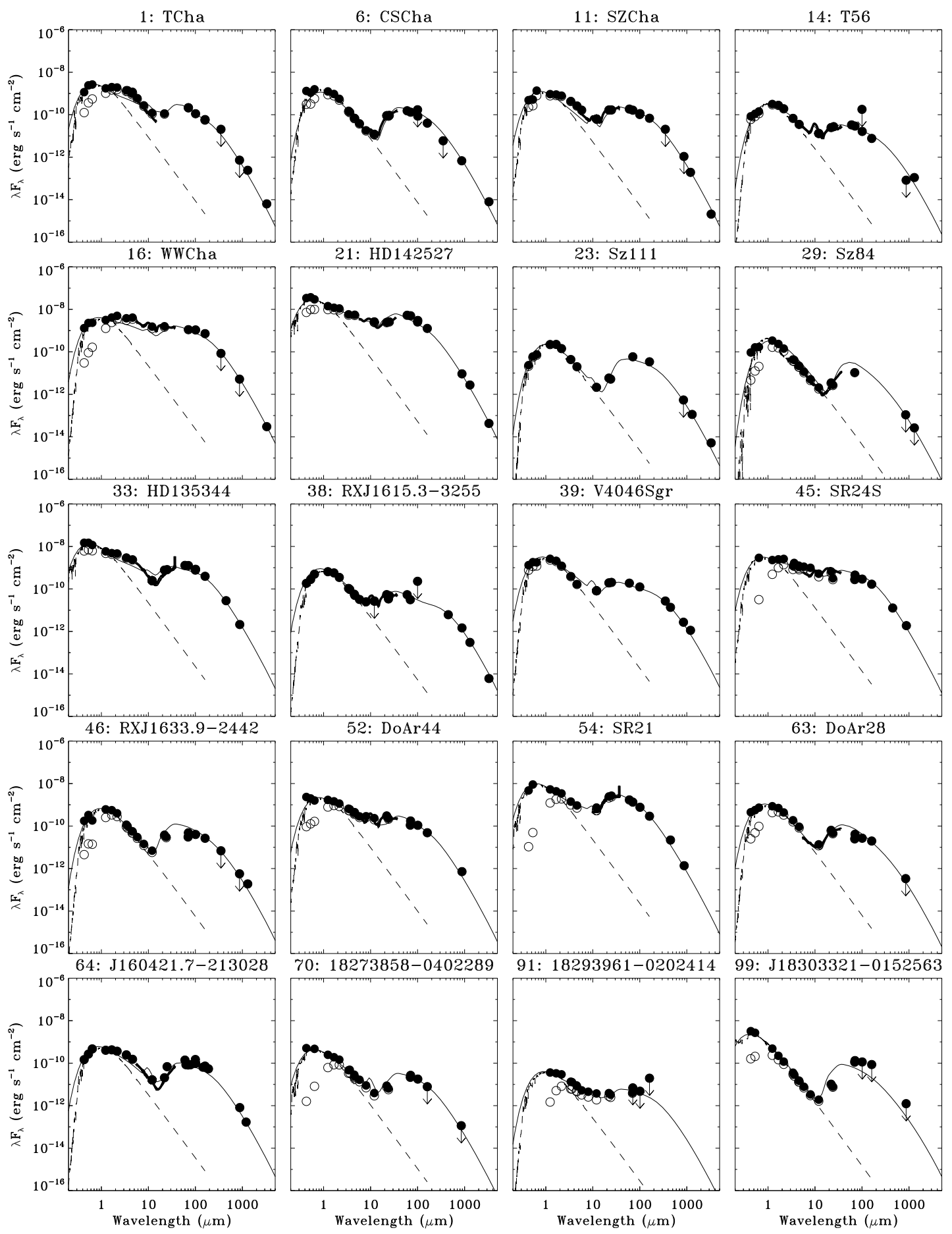

Fig. B.5. SEDs of massive disks with large holes. 
N. van der Marel et al.: The (w)hole survey
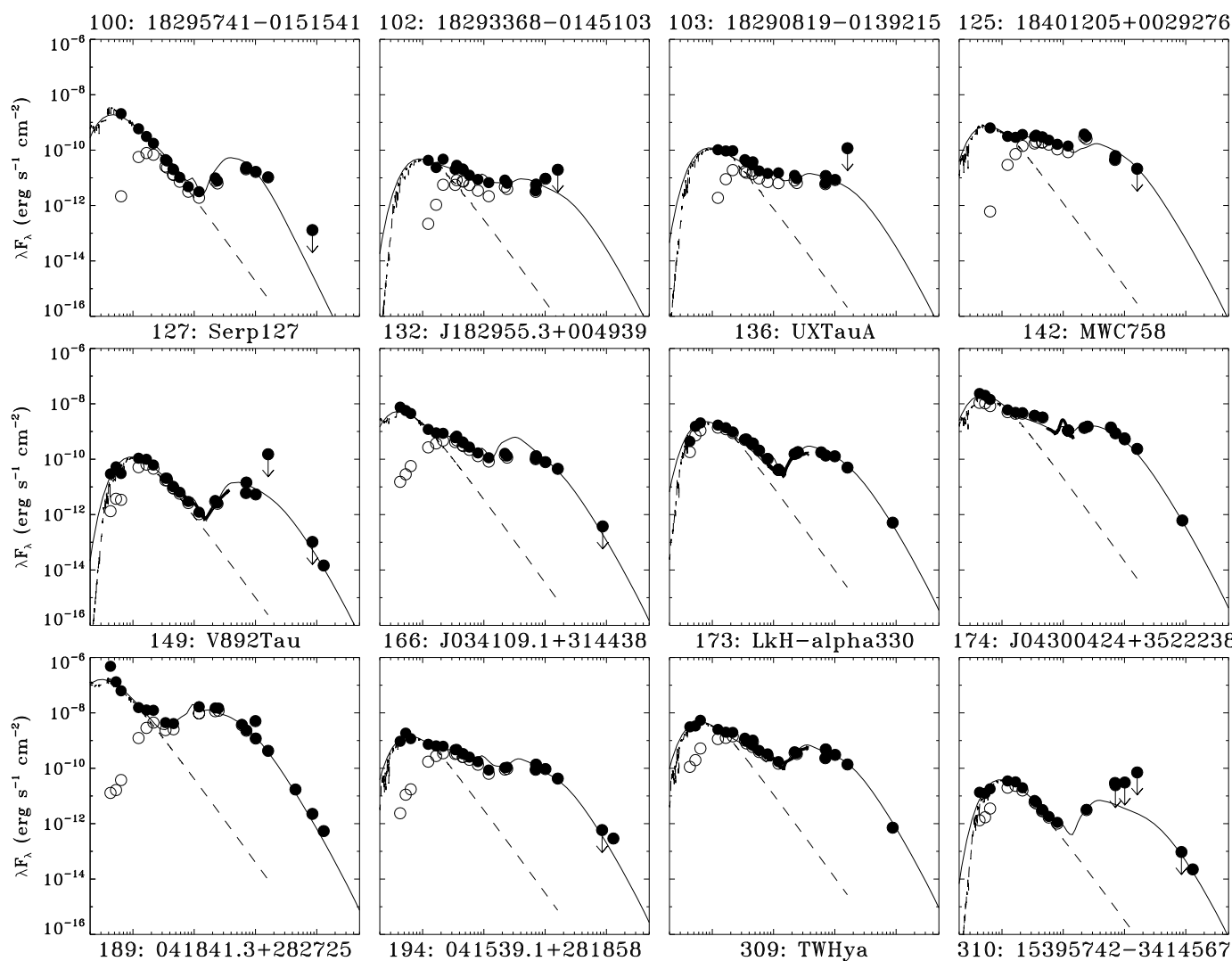

174: J04300424+3522238
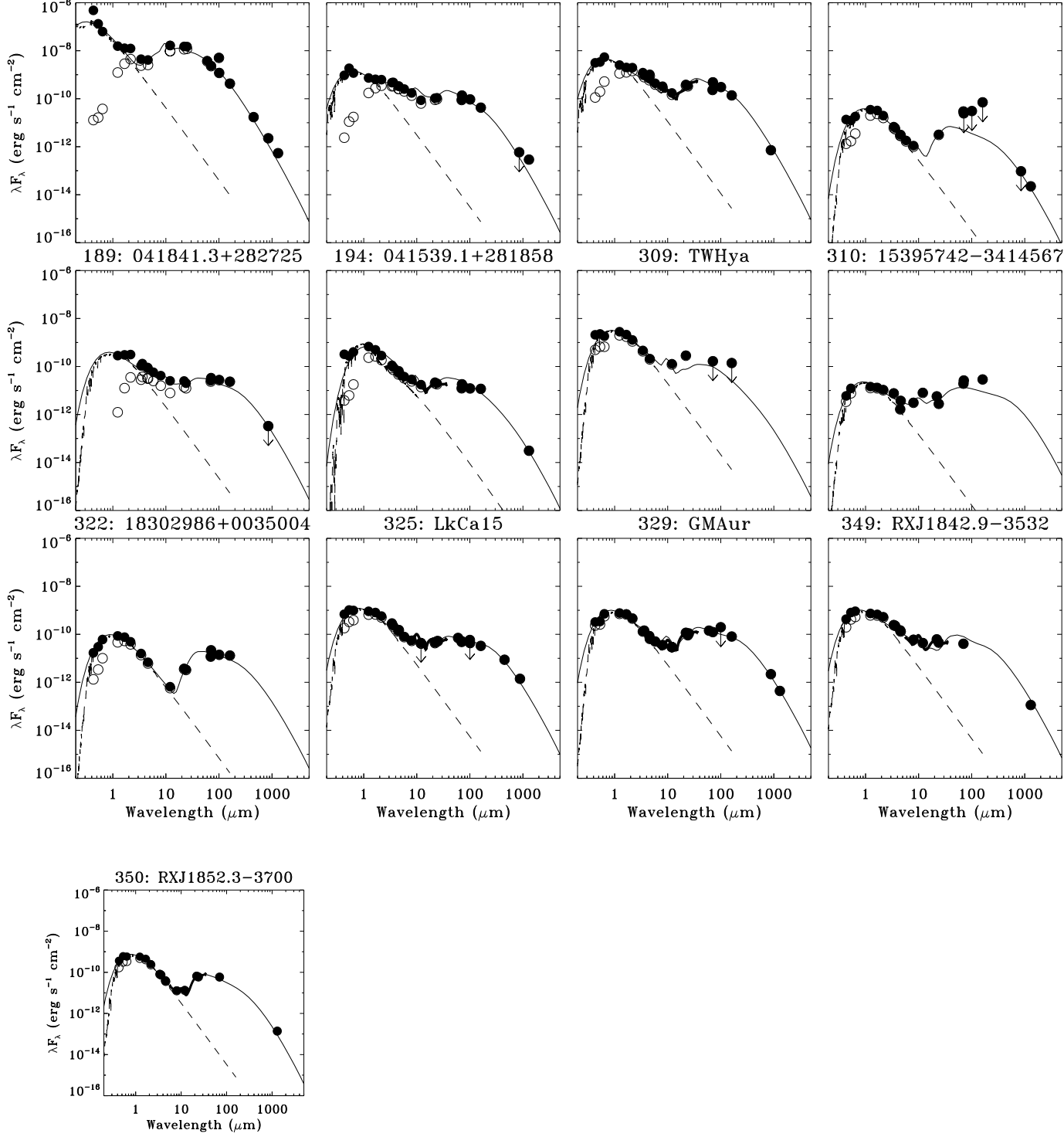

Fig. B.5. continued. 

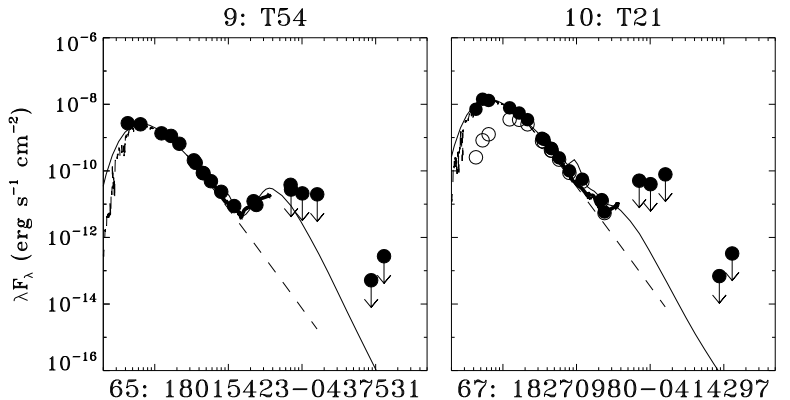

13: ISO-ChaII29

31: 16225309-3724373
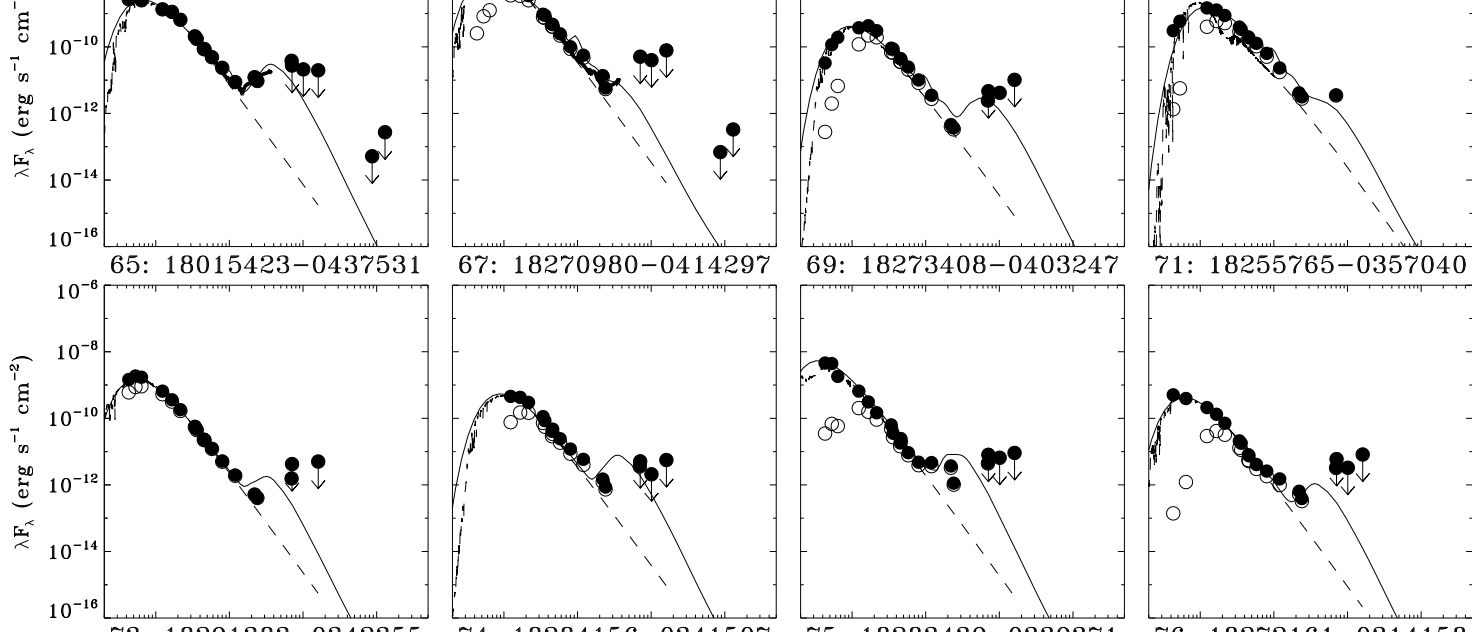

69: $18273408-0403247$
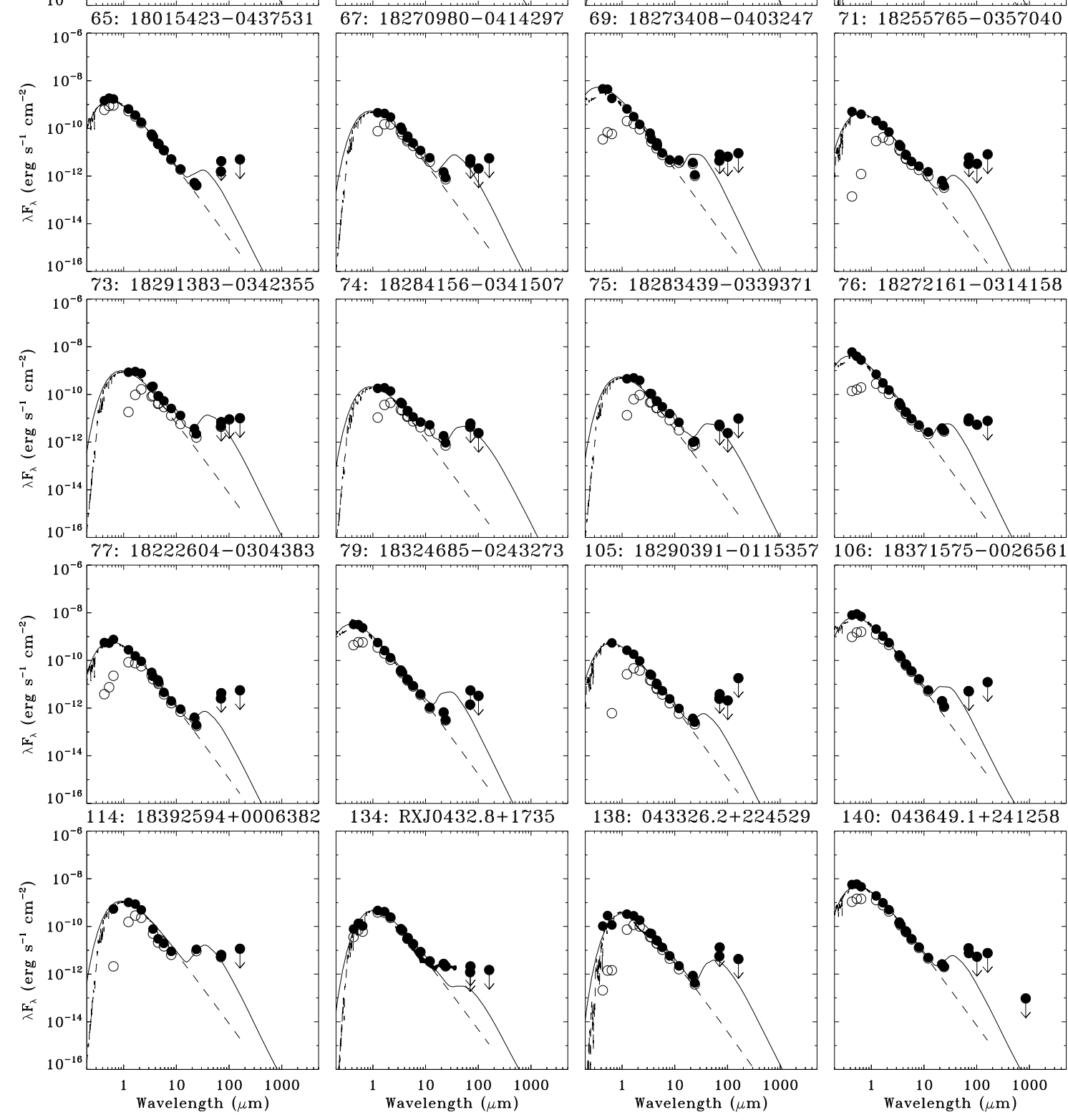

Fig. B.6. SEDs of low scale height disks.

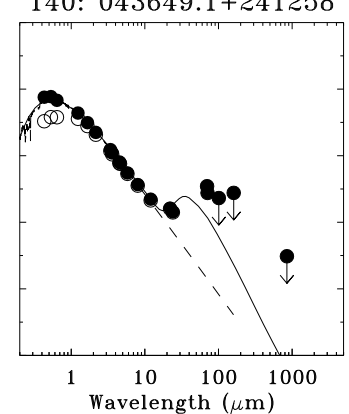



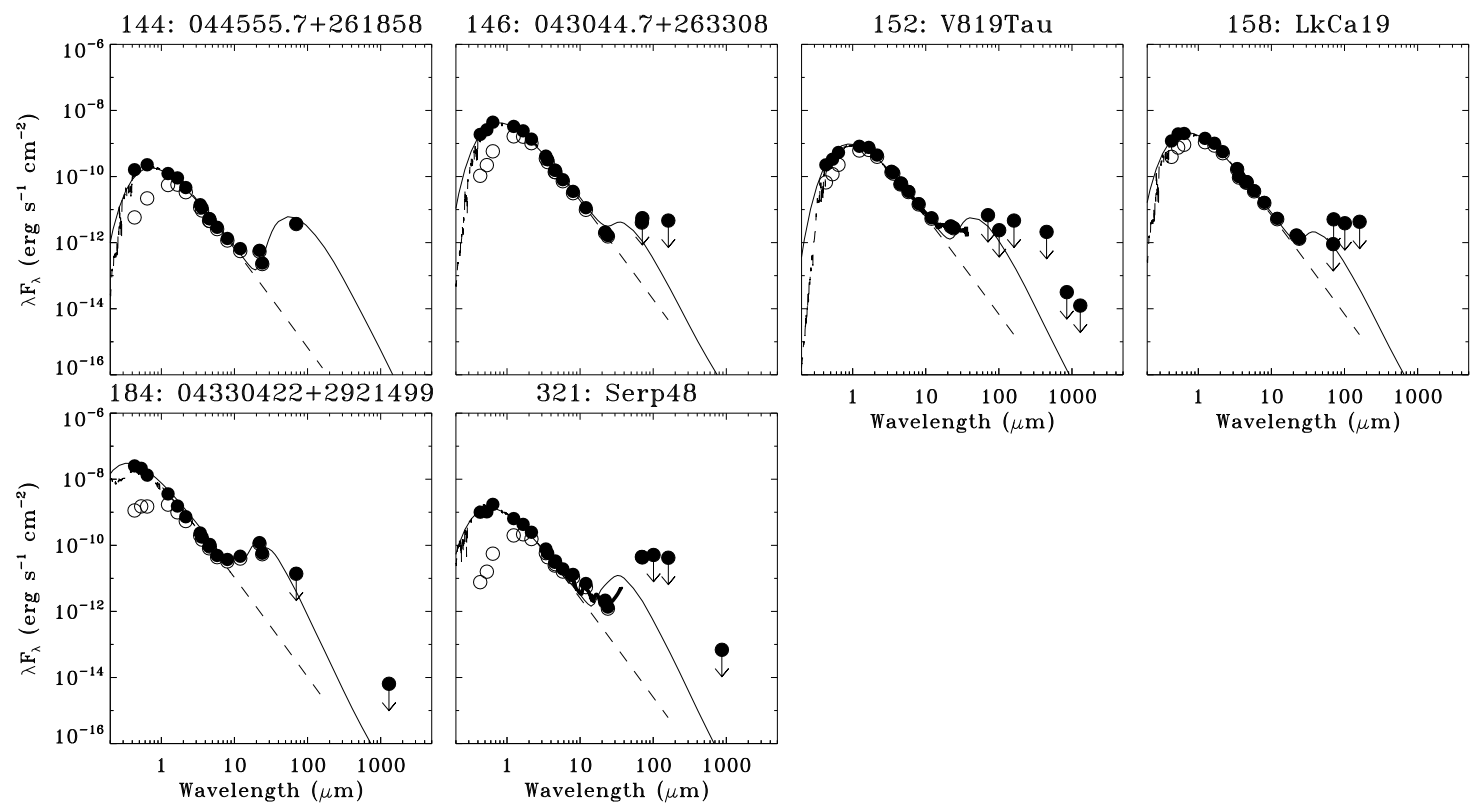

Fig. B.6. continued.

\section{Appendix C: Submillimeter photometry}

Table C.1. Submillimeter photometry.

\begin{tabular}{lllllllll}
\hline \hline ID & $\begin{array}{l}\text { SABOCA } \\
{[350 \mu \mathrm{m}]} \\
(\mathrm{mJy})\end{array}$ & $\begin{array}{l}\text { LABOCA } \\
{[870 \mu \mathrm{m}]} \\
(\mathrm{mJy})\end{array}$ & $\begin{array}{l}\text { SCUBA } \\
{[450 \mu \mathrm{m}]} \\
(\mathrm{mJy})\end{array}$ & $\begin{array}{l}\text { SCUBA } \\
{[850 \mu \mathrm{m}]} \\
(\mathrm{mJy})\end{array}$ & $\begin{array}{l}\text { SMA } \\
{[880 \mu \mathrm{m}]} \\
(\mathrm{mJy})\end{array}$ & $\begin{array}{l}230 \mathrm{GHz} \\
{[1.3 \mathrm{~mm}]} \\
(\mathrm{mJy})\end{array}$ & $\begin{array}{l}110 \mathrm{GHz} \\
{[3.3 \mathrm{~mm}]} \\
(\mathrm{mJy})\end{array}$ & Ref \\
\hline 1 & $2400 \pm 200$ & $210 \pm 20$ & & & & $105 \pm 15$ & $7 \pm 1$ & 1,2 \\
2 & & $<40$ & & & & & 1 \\
6 & $690 \pm 180$ & $197 \pm 12.2$ & & & & & & \\
9 & & $<15$ & & & & $<118$ & & 1,3 \\
10 & $<20$ & & & & & 1,2 \\
$\ldots$ & & & & & & & 1,2 \\
\hline
\end{tabular}

Notes. The full table is available at the CDS.

References. 1) This work. 2) Henning et al. (1993), 3) Lommen et al. (2007).

\section{Appendix D: Herschel photometry}

This section presents the fluxes and cut-out maps of the Herschel PACS photometry.

Table D.1. Herschel photometry.

\begin{tabular}{llll}
\hline \hline ID & $\begin{array}{l}\text { PACS 70 } \\
(\mathrm{mJy})\end{array}$ & $\begin{array}{l}\text { PACS 100 } \\
(\mathrm{mJy})\end{array}$ & $\begin{array}{l}\text { PACS 160 } \\
(\mathrm{mJy})\end{array}$ \\
\hline 1 & $4.98 \pm 0.5$ & $3.74 \pm 0.37$ & $3.17 \pm 0.32$ \\
2 & $0.22 \pm 0.03$ & $0.20 \pm 0.04$ & $<0.27$ \\
3 & $0.11 \pm 0.03$ & $0.14 \pm 0.03$ & $<0.26$ \\
4 & $<0.10$ & $<0.07^{a}$ & $<0.41^{a}$ \\
5 & $0.18 \pm 0.05$ & $0.17 \pm 0.03$ & $<0.33^{a}$ \\
$\ldots$ & & & \\
\hline
\end{tabular}

Notes. The full table is available at the CDS. ${ }^{(a)}$ Upper limit due to cloud confusion. 

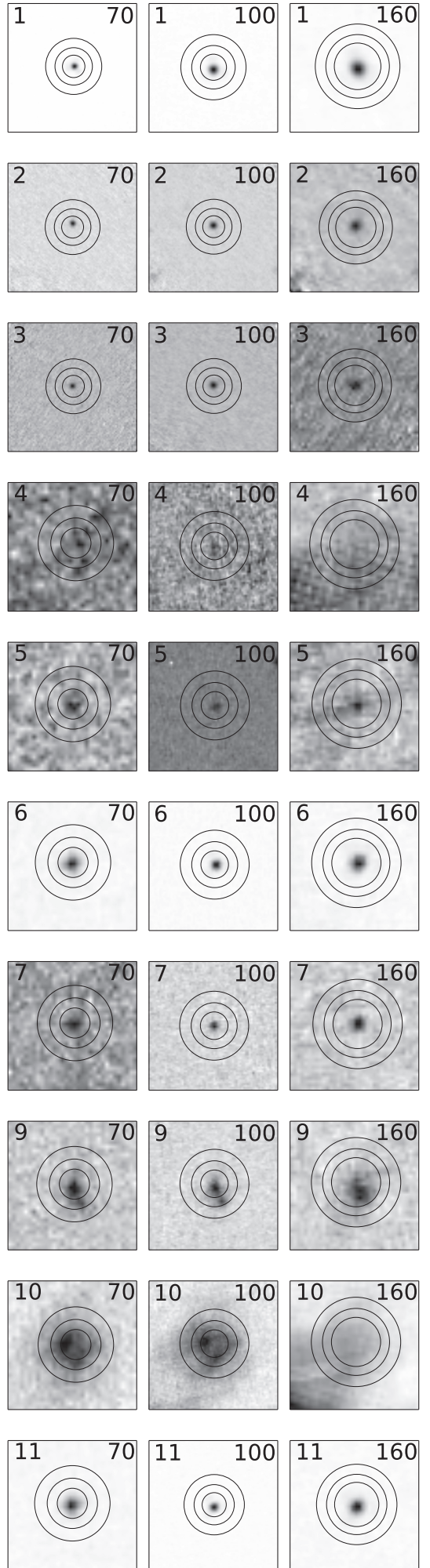
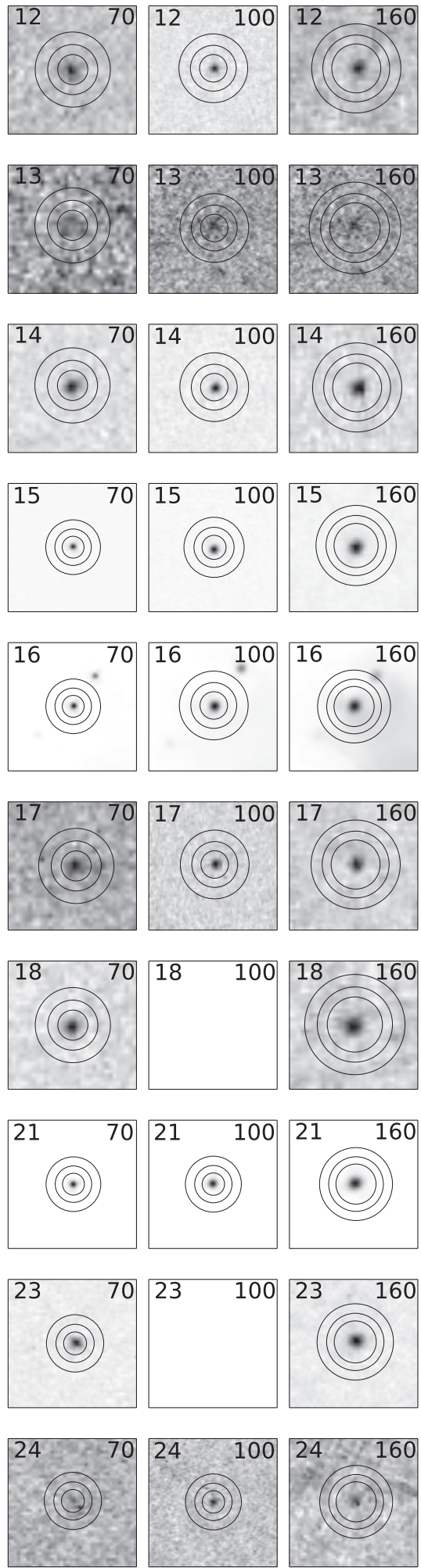

Fig. D.1. Cutouts of the Herschel-PACS maps. The number in the upper left corner corresponds to the ID, the number in the upper right corner corresponds to the wavelength of the image in $\mu \mathrm{m}$. The inner circle indicates the aperture, and the outer two circles denotes the annulus in which in the noise was measured. The color scale has been scaled to the maximum in the image. When no $100 \mu \mathrm{m}$ data is available (see text), the image is left blank. 
N. van der Marel et al.: The (w)hole survey
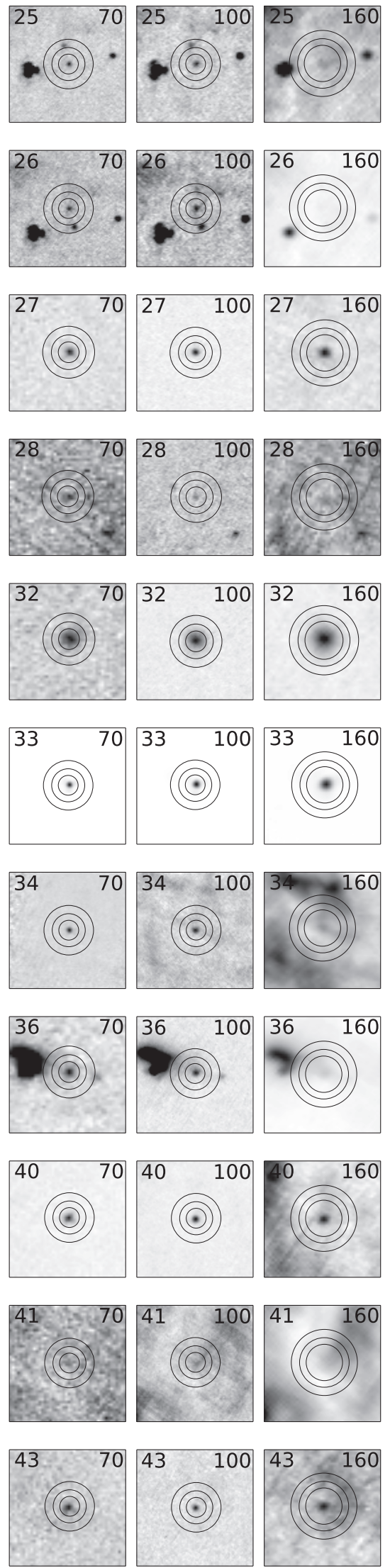
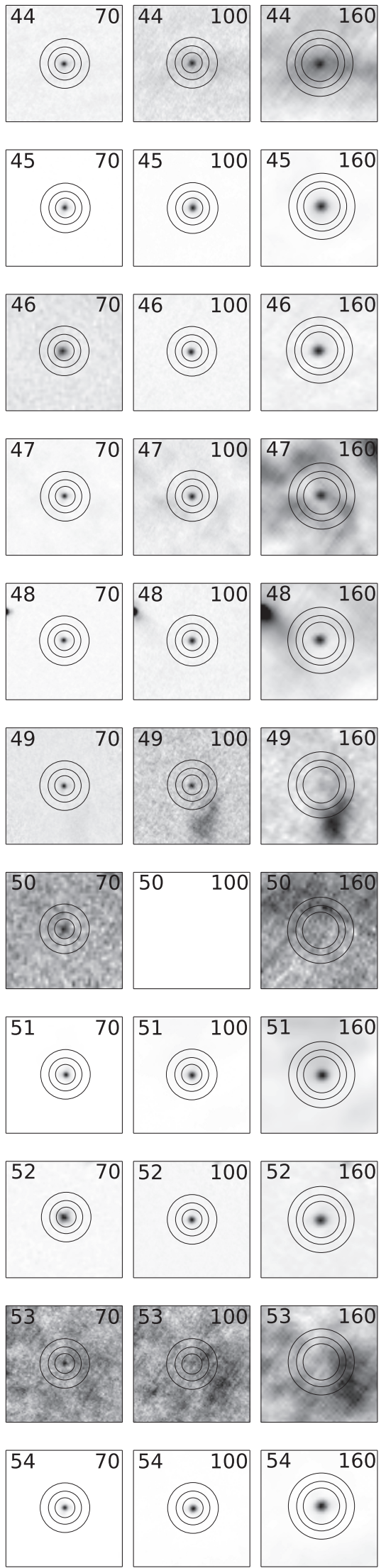

Fig. D.1. continued. 
A\&A 592, A126 (2016)
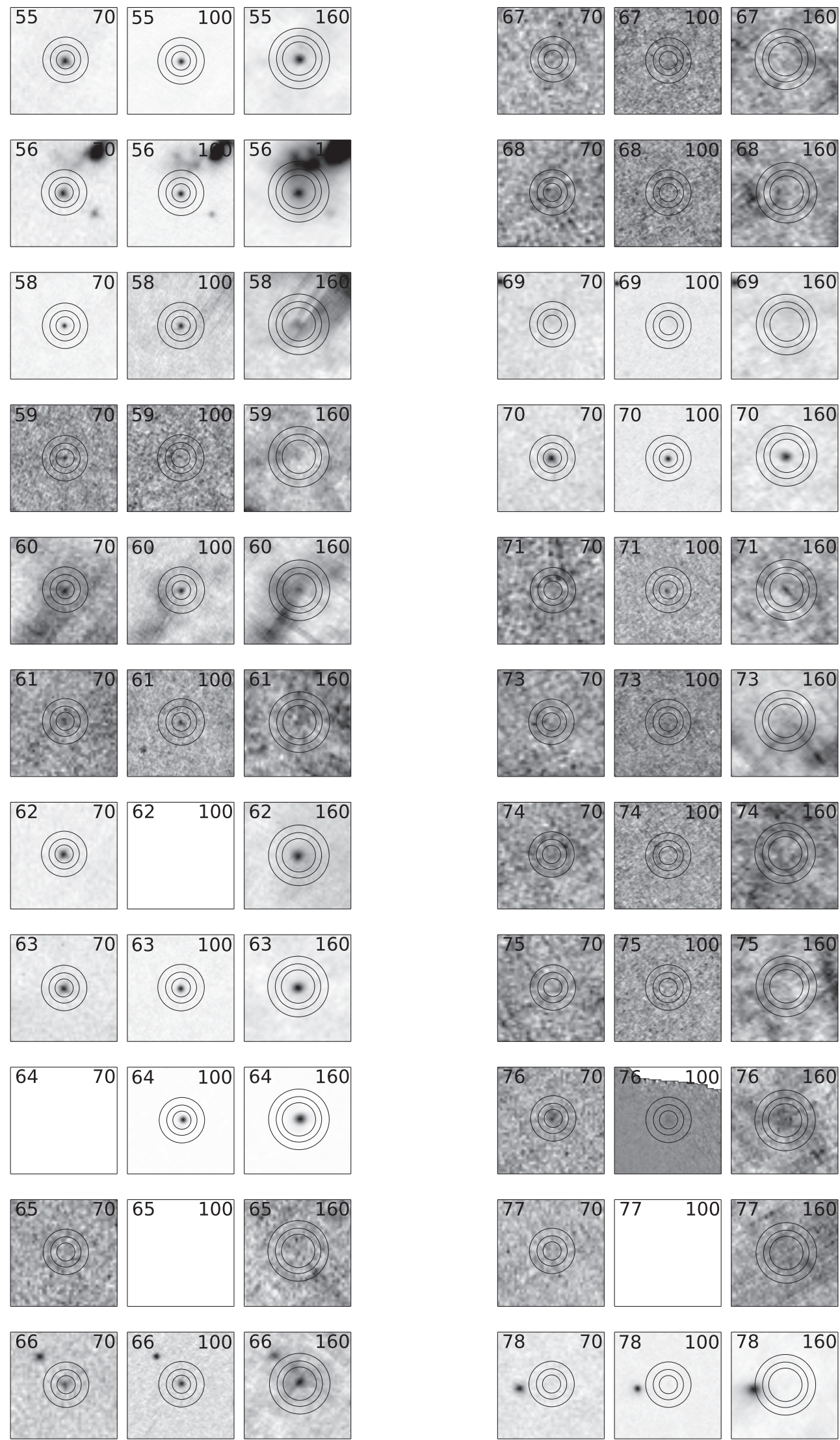

Fig. D.1. continued. 

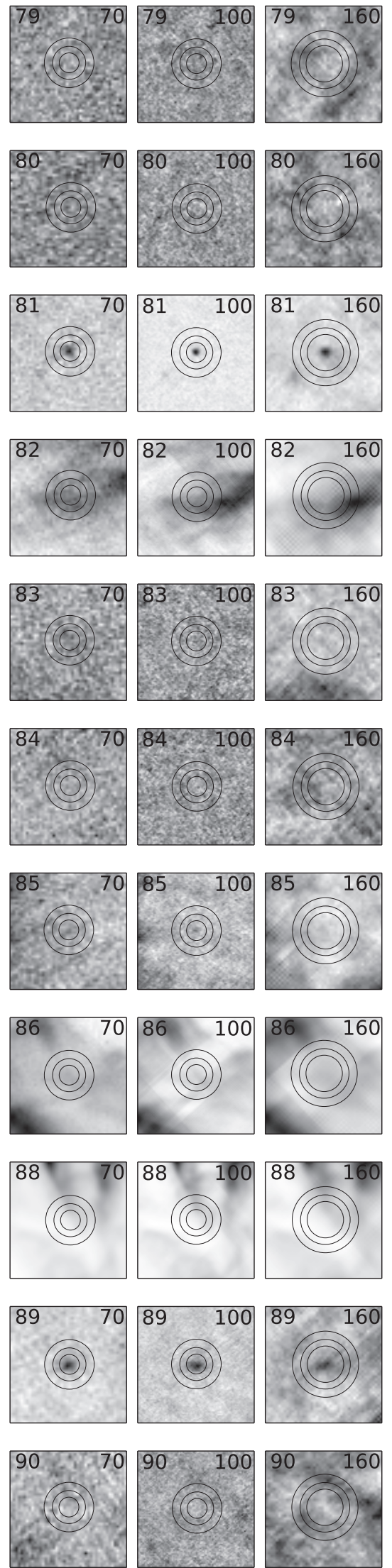
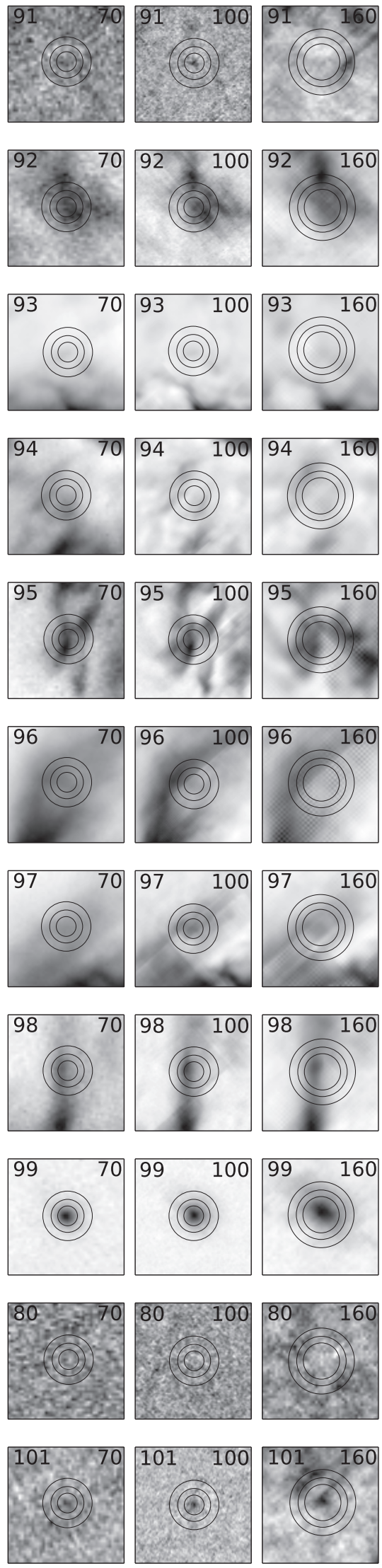

Fig. D.1. continued. 

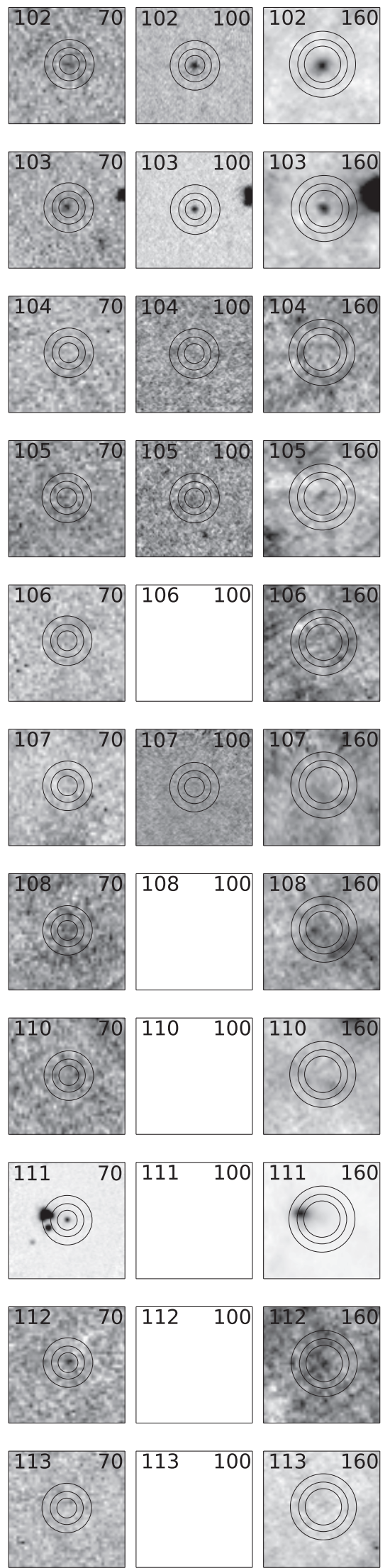
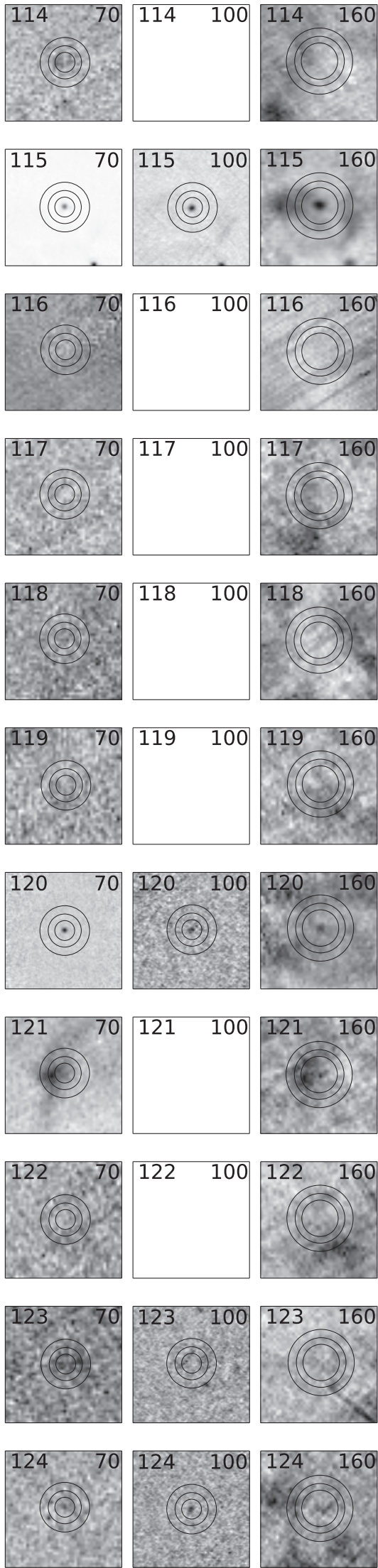

Fig. D.1. continued. 
N. van der Marel et al.: The (w)hole survey
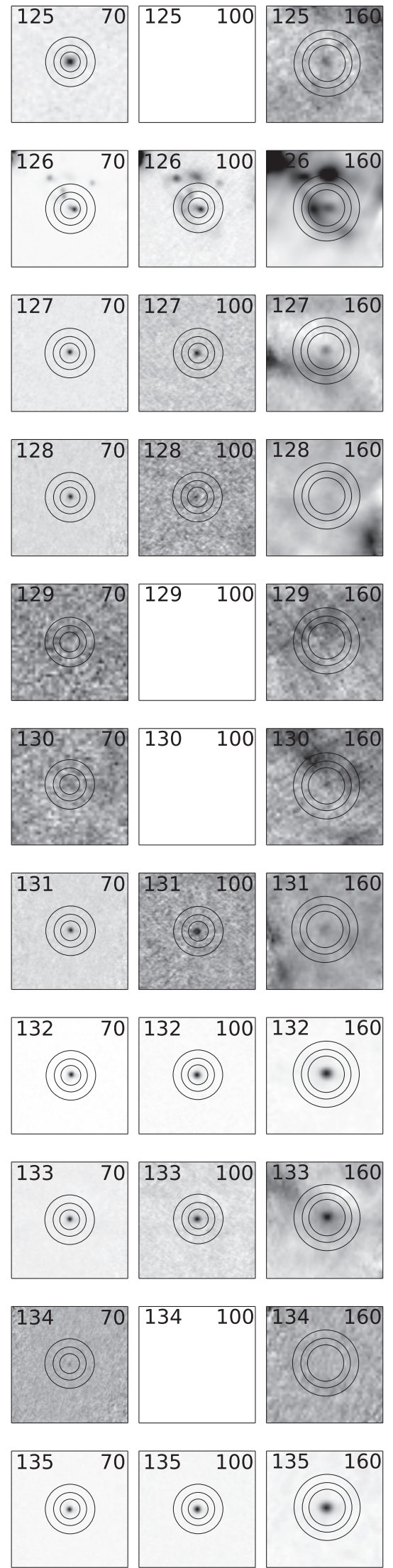
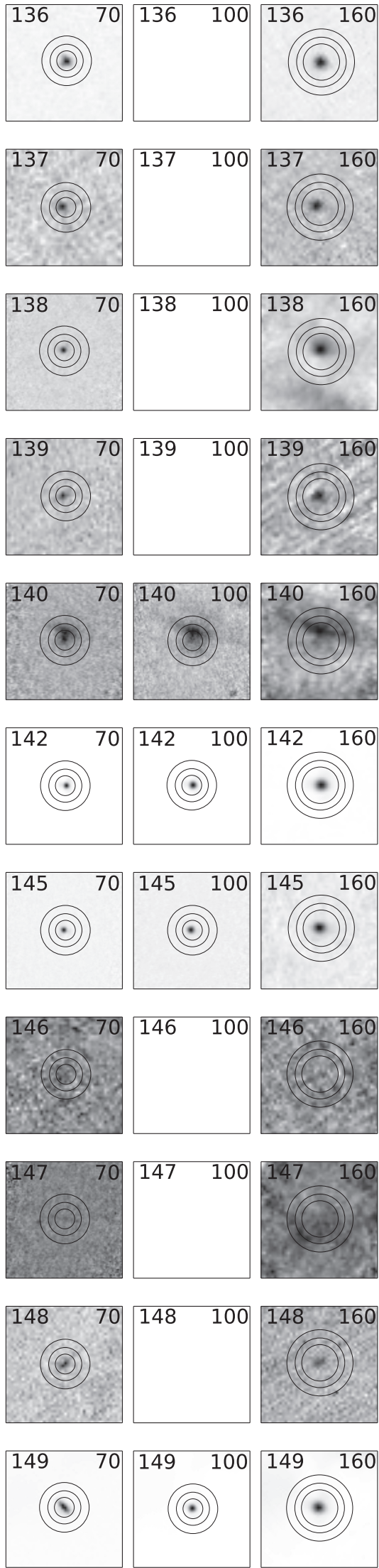

Fig. D.1. continued. 

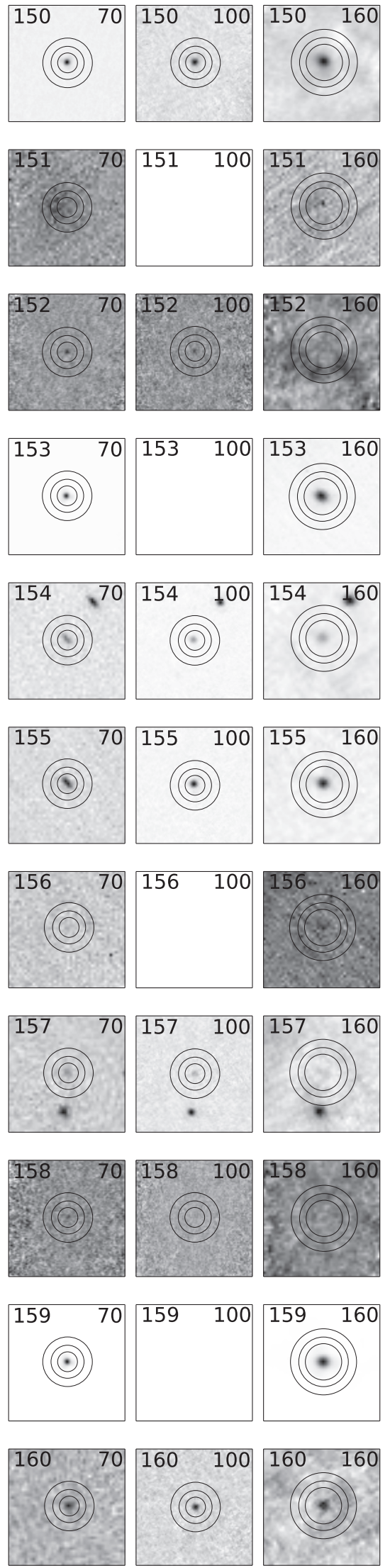
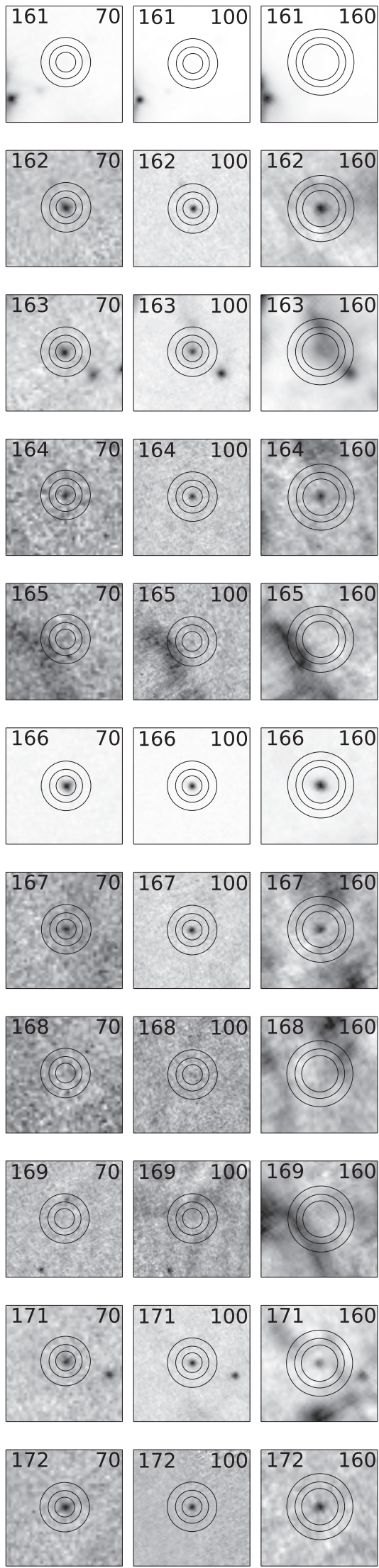

Fig. D.1. continued. 
N. van der Marel et al.: The (w)hole survey
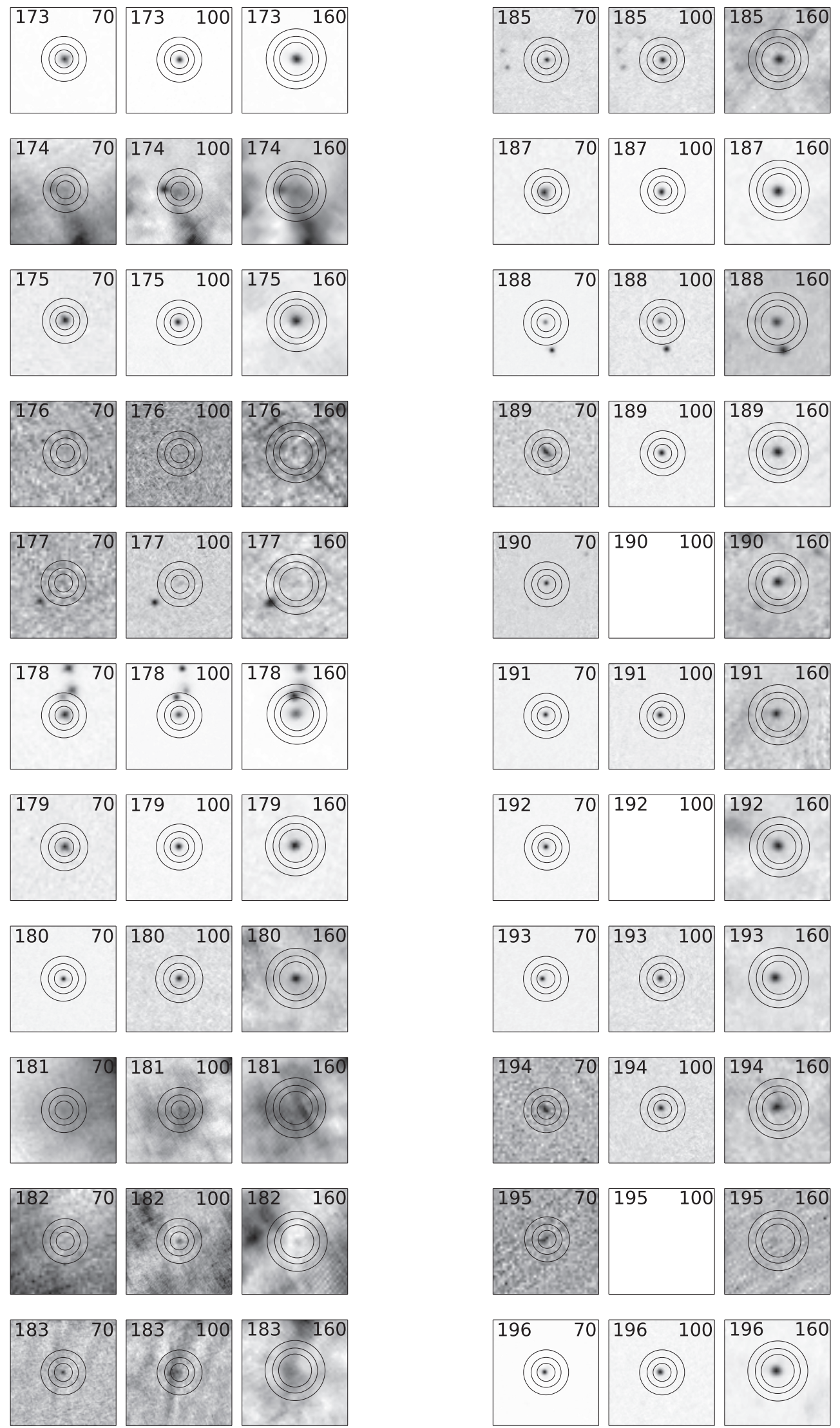

Fig. D.1. continued. 
A\&A 592, A126 (2016)
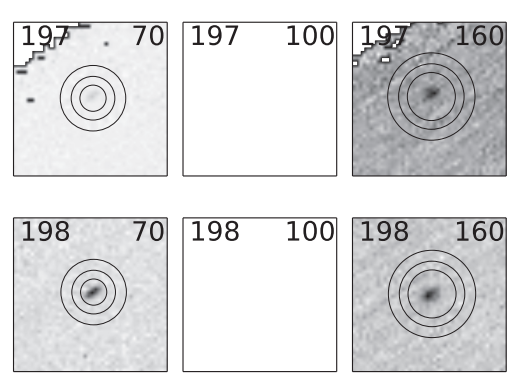

\begin{tabular}{ll|lllll}
200 & 70 & 200 & 100 & 200 & 160
\end{tabular}
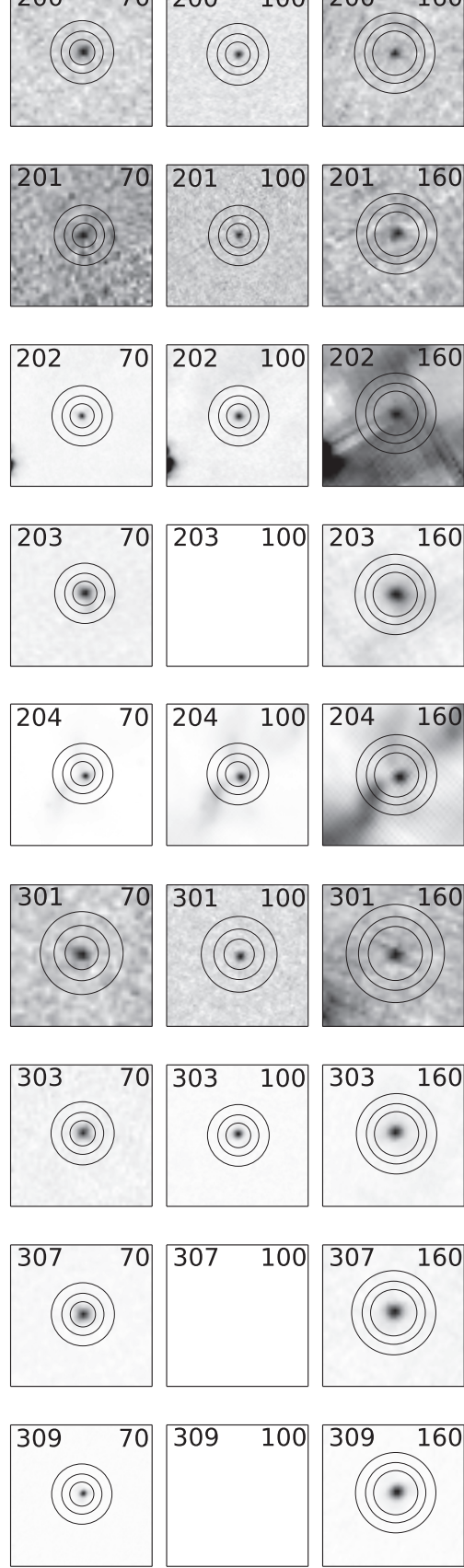
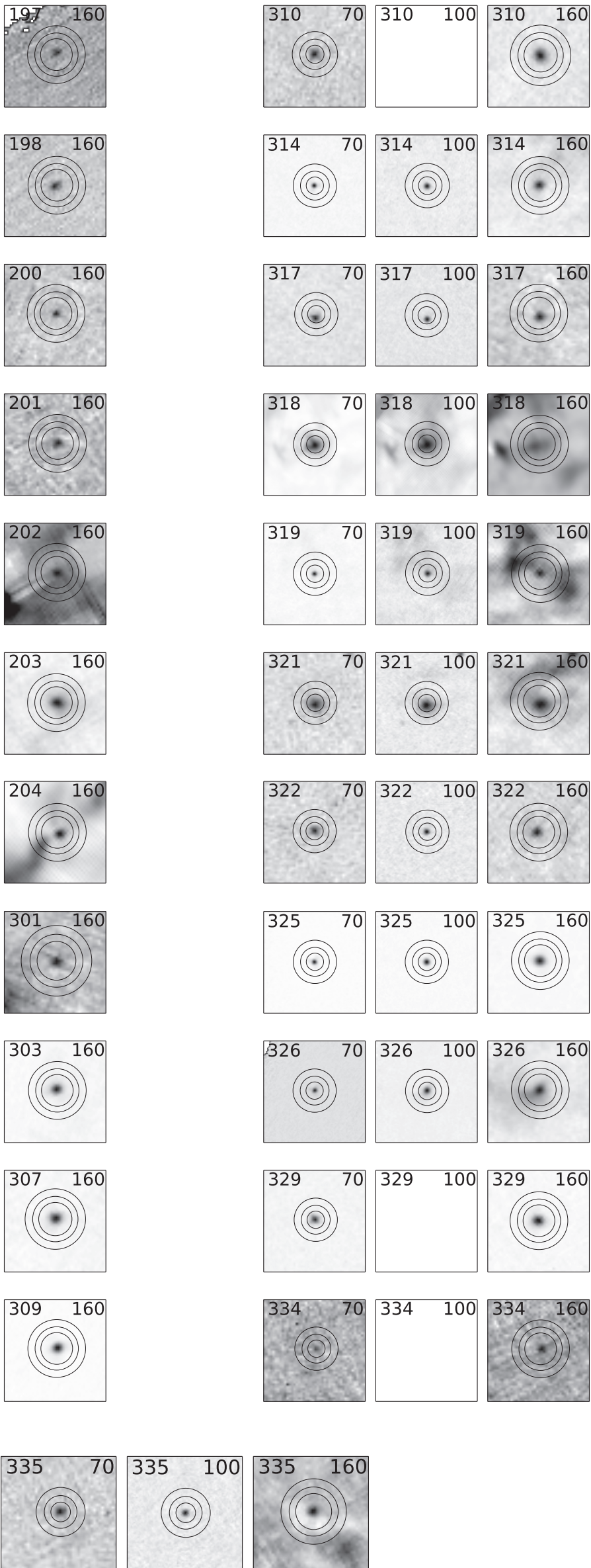

$335 \quad 160$

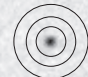

Fig. D.1. continued. 